USP - Universidade de São Paulo

Instituto de Matemática e Estatística

Departamento de Matemática Aplicada

\title{
Seções globais em níveis de energia estritamente convexos com equilibrios do tipo sela-centro \\ para sistemas Hamiltonianos com dois graus de liberdade
}

\author{
Pedro Antonio Santoro Salomão ${ }^{1}$
}

Tese apresentada ao Instituto de Matemática e Estatística da USP para a obtenção do grau de doutor em Matemática Aplicada sob orientação de Clodoaldo Grotta Ragazzo

São Paulo

2002

\footnotetext{
${ }^{\text {I }}$ Durante a elaboração deste trabalho o autor recebeu apoio financeiro da FAPESP
} 
Seções globais em níveis de energia estritamente convexos com equilíbrios do tipo sela-centro para sistemas Hamiltonianos com dois graus de liberdade

Este exemplar corresponde à redação final devidamente corrigida e definida por Pedro Antonio Santoro Salomão e aprovada pela comissão julgadora

São Paulo 2002

\section{Banca Examinadora}

Prof. Dr. Clodoaldo Grotta Ragazzo - IME - USP

Prof. Dr. Daniel Victor Tausk - IME - USP

Prof. Dr. Marco Antonio Teixeira - UNICAMP

Prof. Dr. Marcus Aguiar - UNICAMP

Prof. Dr. Leonardo Magalhães Macarini - IMPA 


\section{Ficha Catalográfica}

Salomão, Pedro Antonio Santoro Salomão

Seções globais em níveis de energia estritamente convexos com equilíbrios do tipo sela-centro para sistemas Hamiltonianos com dois graus de liberdade./ Pedro Antonio Santoro Salomão - - São Paulo, 2002.

$79 \mathrm{p}$. Paulo.

Tese (doutorado) - Instituto de Matemática e Estatística, Universidade de São

1. Sistemas Hamiltonianos. 2. Níveis de energia estritamente convexos. 3. Índice de Conley-Zehnder. 4. Equilíbrios do tipo sela-centro. 5. Seções globais. I. Título. 
Ao meu querido avô

Rodolpho Santoro

(in memorian) 


\section{Agradecimentos}

À toda minha família, principalmente aos meus pais Roberto e Maria Eugênia, à minha avó Lina, à minha irmã Nanda e irmãos Paulo e João, pela paciência durante esse período de dedicação intensa ao trabalho.

Ao professor e amigo Clodoaldo Grotta Ragazzo, que dedicou boa parte de seu tempo para me ajudar a elaborar esse trabalho, contribuindo com grande parte das idéias que foram cruciais para alcançar o objetivo principal da tese, me transmitindo sua ampla experiência como pesquisador e dando estímulos nos momentos mais difíceis.

Ao amigo Salvador Addas Zanata por me incentivar a estudar matemática desde os tempos de faculdade.

Aos professores do IME com quem cursei disciplinas essenciais para o meu aprendizado: Prof a Elza Gomide, Prof a Helena M. A. de Castro, Prof. Jorge Manuel Sotomayor Tello, Prof. Oscar F. V. Erazo, Prof ${ }^{a}$ Mary Lilian Lourenço, Prof a Rosa Maria dos S. B. Chaves, Prof a Élvia M. Sallum, Prof a Iracema M. Bund, Prof. Luiz Antonio Peresi, Prof. Luiz Fichman, Prof. Albert M. Fisher, Prof Edson de Faria e Prof. Sérgio M. Oliva.

Aos amigos do IME, do Colégio Dante Alighieri e da Escola Politécnica.

Ao Professor Daniel V. Tausk, por participar da banca examinadora e me ajudar a entender o conceito de índice de Conley-Zehnder.

Ao Professor Helmut Hofer, que deu boas dicas sobre a caracterização e comportamento da dinâmica em hipersuperfícies convexas.

Aos Professores Leonardo M. Macarini, Marco Antonio Teixeira e Marcus Aguiar, por terem participado da banca examinadora e dado boas sugestões para melhorar a tese e idéias para a continuidade da pesquisa. 
We study the existence of global surfaces of section in non-regular level sets for Hamiltonian systems of two degrees of freedom. We assume that these level sets are strictly convex and its non-regularity is caused by the existence of a saddle-center equilibrium. To make use of an important result of $\mathrm{H}$. Hofer, E. Zehnder and K. Wysocki about the existence of global surfaces of section in regular level sets, we use a hypersurface regularization theorem of M. Ghomi. Properties of Conley-Zehnder index and Moser's normal form of saddle-center equilibria are crucial for the localization of the periodic orbit which is the border of global surfaces of section. A technical hypothesis is necessary to guarantee the existence of a global surface of section in the nonregular case. We present a fast calculation method of the Conley-Zehnder index which can be used numerically. We also verify the convexity hypothesis for Hamiltonian systems extensively studied in dynamical systems literature. 


\section{Resumo}

Estudamos a existência de seções globais em níveis de energia não regulares para sistema Hamiltonianos com dois graus de liberdade. Assumimos que esses níveis de energia são estritamente convexos e perdem a regularidade devido à existência de um equilíbrio do tipo sela-centro. Para utilizarmos um importante resultado de H. Hofer, E. Zehnder e K. Wysocki sobre existência de seções globais em níveis de energia regulares, usamos uma regularização de hipersuperfícies devida a M. Ghomi. Propriedades do índice de ConleyZehnder e uma forma normal de equilíbrios sela-centro devida a J. Moser, desempenham um importante papel para a localização da órbita periódica que é bordo de seções globais. Uma hipótese técnica é necessária para garantirmos a existência de uma seção global no caso não-regular. Apresentamos um método rápido para o cálculo do índice de Conley-Zehnder que pode ser usado numericamente. Verificamos também a hipótese de convexidade em sistemas Hamiltonianos muito estudados na literatura de sistemas dinâmicos. 
Capítulo 1

Capítulo 2 - Hipersuperfícies Regulares em $\mathbb{R}^{4}$ 5

2.1. Geometria Diferencial elementar em $\mathbb{R}^{4}$. .5

2.2. Conjuntos convexos em $\mathbb{R}^{4}$....... .7

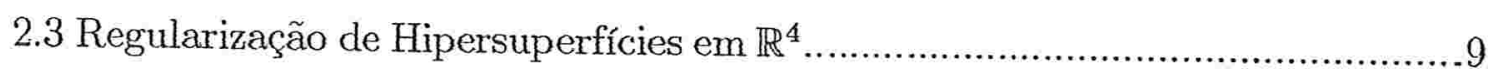

Capítulo 3 - Pontos de equilíbrio do tipo sela-centro............................15

3.1.Perturbações de um Sistema Integrável.........................................................18

3.2. Órbitas Homoclínicas à variedade central de um sela-centro.............................19

Capítulo 4 - O Índice de Conley-Zehnder.........................................22

4.1. Uma Trivialização natural de fibrados lineares

sobre uma Hipersuperfície.

4.2. Definição do índice de Conley-Zehnder

generalizado de órbitas periódicas. .26

4.3. Equivalência do Fluxo Hamiltoniano em Hipersuperfícies Regulares...............29

4.4. Conjuntos invariantes em hipersuperfícies de $\mathbb{R}^{4}$ 33

Capítulo 5 - Estimativa do índice de Conley-Zehnder para órbitas periódicas que passam nas proximidades de um sela-centro............................36

Capítulo 6 - Seções Globais...........................................................44

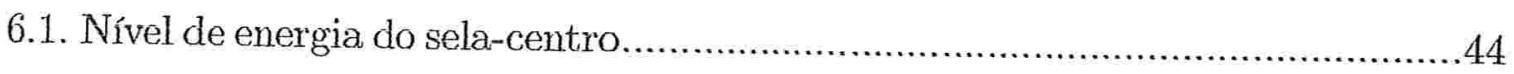

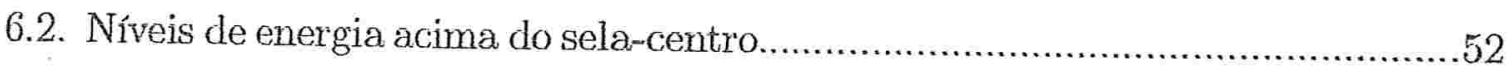


Capítulo 7 - Exemplos e Aplicações.

7.1. Formas Quadráticas Positiva-definidas. .57

7.2. Hamiltonianos da forma $\frac{p_{x}^{2}+p_{y}^{2}}{2}+V(x, y)$. .58

7.3. O Hamiltoniano Henon-Heilles. 61

7.4. Um exemplo integrável. 65

Capítulo 8 - Hipersuperfícies de Contato em $\mathbb{R}^{4}$ 67

8.1. Curvas Pseudo-Holomorfas. .70

8.2. Um sistema de coordenadas importante e a hipótese (H1)...... .73

Bibliografia 


\section{Capítulo 1}

\section{Introdução}

Um dos problemas clássicos sobre sistemas Hamiltonianos ${ }^{1}$ é a existência de órbitas periódicas em determinados níveis de energia. Sabe-se que hipersuperfícies regulares do tipo starshape $^{2}$ sempre apresentam órbitas periódicas (Ver [Rab]). Conseqüentemente, níveis de energia estritamente convexos também possuem órbitas periódicas. Mais ainda, possuem seções globais para o fluxo. Tais seções globais são discos mergulhados cujo bordo é uma órbita periódica com propriedades muito particulares (Ver [HZW]). A existência de seções globais simplifica muito o estudo da dinâmica em tais níveis de energia, pois reduzimos este ao estudo de difeomorfismos em espaços de dimensão menor.

O objetivo principal deste trabalho é estudar a existência de seções globais em níveis de energia estritamente convexos que apresentam um ponto de equilíbrio do tipo sela-centro. Pretendemos estender um resultado de Hofer, Zehnder e Wysocki ([HZW]), dando critérios para existência de seções globais.

Mais precisamente, em [HZW], temos o seguinte

Teorema A. Se $S \subset \mathbb{R}^{4}$ é uma hipersuperfície $C^{2}$, difeomorfa a $S^{3}$ e estritamente convexa, então o fluxo Hamiltoniano associado a $S$ possui uma órbita periódica que é bordo de uma seção global em $S$.

A perda da regularidade de um nível de energia devido à existência de um equilíbrio sela-

\footnotetext{
${ }^{1}$ Consideramos neste trabalho apenas sistemas Hamiltonianos em $\mathbb{R}^{4}$

${ }^{2} U m$ hipersuperfície $S \subset \mathbb{R}^{4}$, compacta e difeomorfa a $S^{3}$ é do tipo star-shape (estrelada) se existir um ponto $p$ pelo qual toda semi-reta que começa em $p$ intersecta $S$ em exatamente 1 ponto.
} 
centro impede o uso do Teorema de Hofer-Zehnder-Wysocki. Com uma hipótese técnica adicional ( $H 1)$, definida em (6.4), provamos o seguinte

Teorema B. Seja $H: \mathbb{R}^{4} \rightarrow \mathbb{R}$ uma função real-analítica. Seja $S \subset H^{-1}(0)$ uma hipersuperfície estritamente convexa, homeomorfa a $S^{3}$, regular em todo ponto exceto em $p_{c} \in S$, que corresponde a um equilíbrio do tipo sela-centro. Suponhamos que vale a hipótese $(H 1)$. Então o fluxo Hamiltoniano associado à função $H$ possui uma órbita periódica em $S$ que é bordo de uma seçầo global em $S$.

É muito comum estudarnos os níveis de energia próximos ao do sela-centro. Um corolário importante da existência de seções globais é a existência de uma infinidade de órbitas periódicas e de órbitas homoclínicas à variedade central do sela-centro. Para isso, utilizamos técnicas semelhantes às usadas em [BGS].

A estratégia para a demonstração desse resultado é apresentada a seguir:

O nível de energia que contém o sela-centro pode ser regularizado numa vizinhança arbitrariamente pequena do equilíbrio utilizando um resultado de M. Ghomi ([Gho]). Para isso, algumas hipóteses sobre a convexidade do nível de energia devem ser verificadas.

Feita a regularização, sabemos que a nova hipersuperfície regularizada possui seção global em forma de disco. Surgem então 2 perguntas:

(i) A órbita periódica que é o bordo dessa seção global passa longe da região onde foi feita a regularização?

(ii) Se (i) for verdadeira, o disco mergulhado que representa a seção global intersecta a região onde foi feita a regularização?

Responderemos a primeira pergunta afirmativamente e daremos um critério para uma resposta afirmativa da segunda pergunta. Neste caso, podemos provar a existência de seções globais para o caso da hipersuperfície com o sela-centro.

A estrutura do trabalho foi organizada da seguinte forma:

Inicialmente, apresentaremos algumas definições elementares de hipersuperfícies regulares em $\mathbb{R}^{4}$ e alguns conceitos de geometria diferencial como aplicação normal de Gauss, aplicação de Weingarten e curvatura seccional.

Caracterizaremos conjuntos convexos e estritamente convexos em $\mathbb{R}^{4}$, estabelecendo uma relação entre convexidade estrita e curvatura para hipersuperfícies com um ponto crítico. Essa 
caracterização será fundamental para podermos regularizar uma hipersuperfície, conhecendo-se apenas suas propriedades geométricas locais.

Apresentaremos uma forma normal dos equilíbrios do tipo sela-centro devida a J. Moser (ver [Mo]). Mostraremos também alguns resultados sobre a existência de órbitas homoclínicas à variedade central de um sela-centro para perturbações de sistemas integráveis. Ver mais detalhes em [BGS].

Serão apresentadas duas definições do indice de Conley-Zehnder generalizado de órbitas periódicas (Ver [CZ] e [HZW]). A primeira delas tem um caráter espectral e é geral. Já a segunda utiliza conceitos geométricos. Daremos enfoque principalmente à definição geométrica, que apesar de estar definida apenas para órbitas periódicas não-degeneradas, fornece uma estimativa muito boa para as órbitas periódicas degeneradas.

Apresentaremos um método rápido para o cálculo do índice de Conley-Zehnder de órbitas periódicas. O índice de Conley-Zehnder e a forma normal de Moser serão fundamentais para obtermos esses resultados. Mais precisamente, sabemos que o índice do bordo da seção global da hipersuperfície regularizada é 3 , e isso nos permite mostrar que tal órbita periódica não pode estar na região onde foi feita a regularização.

Estabeleceremos uma trivialização do fibrado tangente de uma hipersuperfície em $\mathbb{R}^{4}$ apresentada em [CPR]. As equações do fluxo linearizado nesse referencial trivializado apresentarn características geométricas muito importantes para mostrarmos que órbitas periódicas com índice de Conley-Zehnder limitado não podem passar arbitrariamente próximas de um equilíbrio do tipo sela-centro.

Apresentaremos uma definição natural de seção global para hipersuperfícies não regulares, no caso particular em que a perda de regularidade ocorre devido à existência de um equilíbrio sela-centro. Tais equilíbrios apresentam variedades estável e instável uni-dimensionais e é natural pensarmos que tais órbitas estão conectadas pelo fluxo.

Apresentaremos um método simples para verificação de convexidade estrita em níveis de energia para Hamiltonianos do tipo "energia cinética + potencial" e verificaremos a convexidade em alguns casos como por exemplo o Hamiltoniano Henon-Heilles, muito estudado na literatura de sistemas mecânicos.

Finalmente, daremos também uma introdução à hipersuperfícies de contato em $\mathbb{R}^{4}$ assim 
como as propriedades de curvas pseudo-holomorfas. O objetivo principal desta parte é preparar algumas ferramentas para a eliminação da hipótese técnica $(H 1)$ e, com isso, generalizar o Teorema B. 


\section{Capítulo 2}

\section{Hipersuperfícies Regulares em $\mathbb{R}^{4}$}

\subsection{Geometria Diferencial elementar em $\mathbb{R}^{4}$}

Um subconjunto $S$ contido em $\mathbb{R}^{4}$ é uma hipersuperfície regular $C^{k}$ se para todo ponto $p \in S$ existirem abertos $U \subset \mathbb{R}^{3}$ e $V \subset \mathbb{R}^{4}$ e uma aplicação $X: U \rightarrow V \cap S$ com as seguintes propriedades:

(1) $X$ é diferenciável $\left(C^{k}\right)$;

(2) $X$ é homeomorfismo;

(3) Para todo $q \in U, d X(q)$ é injetora;

A aplicação $X$ é chamada parametrização ou sistema de coordenadas numa vizinhança de $p$ e a aplicação $X^{-1}$ é chamada de carta. O conjunto $V \cap S$ é chamado de vizinhança coordenada.

Os exemplos mais comuns de hipersuperfícies em $\mathbb{R}^{4}$ são:

(1) Gráfico de uma função diferenciável. Seja $g: U \subset \mathbb{R}^{3} \rightarrow \mathbb{R}$ uma função diferenciável, onde $U$ é um conjunto aberto. Então $S_{g}=\left\{\left(x_{1}, x_{2}, x_{3}, x_{4}\right) \in \mathbb{R}^{4} \mid\left(x_{1}, x_{2}, x_{3}\right) \in U, x_{4}=\right.$ $\left.g\left(x_{1}, x_{2}, x_{3}\right)\right\}$ é uma hipersuperfície regular. Uma parametrização global para $S_{g}$ é dada por $X_{g}: U \subset \mathbb{R}^{3} \rightarrow S_{g} \subset \mathbb{R}^{4}$ onde $X_{g}(u, v, w)=(u, v, w, g(u, v, w))$.

(2) Hipersuperfície definida implicitamente como imagem inversa de um valor regular: Seja $H: \mathbb{R}^{4} \rightarrow \mathbb{R}$ uma função diferenciável. Um ponto $x_{0} \in \mathbb{R}^{4}$ é chamado de ponto crítico se $d H\left(x_{0}\right)$ não é sobrejetora, ou seja, se todas as derivadas parciais de $H$ se anulam em $x_{0}$. Caso contrário $x_{0}$ é chamado de ponto regular. Um ponto $t_{0} \in \mathbb{R}$ é chamado de valor regular se o conjunto $H^{-1}\left(t_{0}\right)$ não contém pontos críticos. Então, para todo $t \in \mathbb{R}$ regular de $H, H^{-1}(t)$ é 
uma hipersuperfície regular. Por exemplo, se $H\left(x_{1}, x_{2}, x_{3}, x_{4}\right)=x_{1}^{2}+x_{2}^{2}+x_{3}^{2}+x_{4}^{2}-1$ então 0 é um valor regular de $H$ e $H^{-1}(0)=S^{3}$, chamada de esfera unitária de dimensão 3 , é uma hipersuperfície regular.

Uma aplicação $\alpha: I \subset \mathbb{R} \rightarrow S \subset \mathbb{R}^{3}$ diferenciável é chamada de curva na hipersuperfície $S$. Um vetor $v \in T_{p} \mathbb{R}^{4} \simeq \mathbb{R}^{4}$ é um vetor tangente a $S$ em $p$, se existe uma curva diferenciável $\alpha:(-\varepsilon, \varepsilon) \rightarrow S$ tal que $\alpha(0)=p$ e $\alpha^{\prime}(0)=v$. O conjunto de todos os vetores tangentes a $S$ em $p$ é chamado de espaço tangente a $S$ em $p$, e denotado por $T_{p} S$. Um vetor normal a $S$ em $p$ é um vetor $N \in \mathbb{R}^{4}$ tal que $N \perp T_{p} S$, ou seja, $\langle w, N\rangle=0$ para todo $w \in T_{p} S$.

Se $X: U \subset \mathbb{R}^{3} \rightarrow S \subset \mathbb{R}^{4}$ é uma parametrização de uma hipersuperfície regular $S$ numa vizinhança de $p \in S$ onde $p=X(q)$, então $d X(q)\left(T_{q} \mathbb{R}^{3}\right)=T_{p} S$ e, portanto, $\operatorname{dim} T_{p} S=3$.

Chamamos de fibrado tangente a $S$ o conjunto $T S \stackrel{\text { def }}{=} \cup_{q \in S} T_{q} S=\left\{(x, v) \mid x \in S\right.$ e $\left.v \in T_{x} S\right\}$. $T S$ é localmente homeomorfo a $U \times \mathbb{R}^{3}$ onde $U \subset \mathbb{R}^{3}$ é um subconjunto aberto. O fibrado normal a $S$ é dado por $N S \stackrel{\text { def }}{=}\left\{(x, v) \mid x \in S\right.$ e $\left.v \in\left(T_{x} S\right)^{\perp}\right\}$ e localmente é homeomorfo a $U \times \mathbb{R}$.

Um campo de vetores tangente a uma hipersuperfície regular $S$ é uma aplicação $W: S \rightarrow T S$ tal que para todo $p \in S, W(p)=(p, v)$ onde $v \in T_{p} S$. Omitiremos a $1^{a}$ componente (identidade em $S$ ) do campo de vetores $W$. Por exemplo, dada uma parametrização $X: U \subset \mathbb{R}^{3} \rightarrow$ $S \subset \mathbb{R}^{4}$, a aplicação $X_{u}: U \rightarrow T S$ dada por $X_{u}(q) \stackrel{\text { def }}{=} \frac{\partial X}{\partial u}(q)$ define um campo de vetores tangente a $S$ em $X(U)$. De forma análoga podemos definir campos de vetores associados às aplicações $X_{v}$ e $X_{w}$ onde $(u, v, w)$ são as coordenadas em $U$. Um campo de vetores $W$ é dito diferenciável em $p \in S$ se para alguma parametrização $X$ de uma vizinhança de $p$ temos $W(q)=a(q) X_{u}(q)+b(q) X_{v}(q)+c(q) X_{w}(q)$ onde $a, b$ e $c$ são funções diferenciáveis a valores reais em $U$. O campo de vetores $W$ é diferenciável em $S$ se $W$ for diferenciável em todo $p \in S$.

Dada $X: U \rightarrow S$ uma parametrização de $S$, podemos definir um campo de vetores normais unitários em $X(U)$ pela aplicação $N: U \rightarrow N S$ dada por $N(q) \stackrel{\text { def }}{=} \frac{X_{u}(q) \wedge X_{v}(q) \wedge X_{w}(q)}{\left\|X_{u}(q) \wedge X_{v}(q) \wedge X_{w}(q)\right\|}$ onde $a \wedge b \wedge c$ é o produto exterior dos vetores $a, b$ e $c$ definido implicitamente por $\operatorname{det}(a, b, c, x)=$ $\langle a \wedge b \wedge c, x\rangle$ para todo $x \in \mathbb{R}^{4}$. Uma hipersuperfície regular $S$ é orientável se e somente se existe um campo contínuo de vetores normais em $S$. A esfera $S^{3}$ é um exemplo de hipersuperfície orientável, onde $N: S^{3} \rightarrow N S^{3} \cong S^{3}, N(x)=x$ é uma orientação para $S^{3}$.

Se $S$ é uma hipersuperfície regular dada por $H^{-1}(c)$ onde $H: \mathbb{R}^{4} \rightarrow \mathbb{R}$ é diferenciável e $c$ é um valor regular de $H$, então para todo $p \in S, T_{p} S=\operatorname{ker} d H(p)=\left[H_{x}(p)\right]^{\perp}=[N(p)]^{\perp}$. 
Dado um ponto $p \in S$, podemos identificar $T_{p} S$ e $T_{N(p)} S^{3}$ pois $T_{N(p)} S^{3}=[N(p)]^{\perp}=T_{p} S$. Desta forma, seja $d N(p): T_{p} S \rightarrow T_{p} S$ a diferencial de $N$ no ponto $p$. A aplicação $d N(p)$ é linear e auto-adjunta, ou seja, $\langle d N(p) v, w\rangle=\langle v, d N(p) w\rangle$ para todo $v, w \in T_{p} S$. Portanto, existe uma base ortonormal $\left\{e_{1}(p), e_{2}(p), e_{3}(p)\right\}$ de $T_{p} S$ tal que $d N(p) e_{i}(p)=-k_{i}(p) e_{i}(p), i=1,2,3$, onde $k_{i}$ são números reais chamados de curvaturas principais de $S$.

Dizemos que uma hipersuperfície $S$ tem curvatura positiva em $p \in S$ se $k_{i}(p) k_{j}(p)>0$ para todo $1 \leqq i, j \leqq 3$. Uma hipersuperfície $S$ tem curvatura positiva se $S$ tem curvatura positiva em todo $p \in S$. Os valores $k_{i}(p) k_{j}(p)$ para $1 \leqq i \neq j \leqq 3$ são chamados de curvaturas seccionais de $e_{i}(p)$ e $e_{j}(p)$.

Dados dois vetores ortonormais $X_{1}(p)$ e $X_{2}(p)$ contidos em $T_{p} S$, então $K_{X_{1} X_{2}}$, a curvatura seccional associada ao plano gerado por $X_{1}(p)$ e $X_{2}(p)$, é dada por

$$
K_{X_{1} X_{2}} \stackrel{\text { def }}{=} \operatorname{det}\left(\begin{array}{ll}
\left\langle d N(p) X_{1}, X_{1}\right\rangle & \left\langle d N(p) X_{1}, X_{2}\right\rangle \\
\left\langle d N(p) X_{1}, X_{2}\right\rangle & \left\langle d N(p) X_{2}, X_{2}\right\rangle
\end{array}\right)
$$

e é positiva se $S$ tiver curvatura positiva em $p$. Para maiores informações sobre hipersuperfícies em $\mathbb{R}^{4}$, ver [Tho].

\subsection{Conjuntos convexos em $\mathbb{R}^{4}$}

Dizemos que um subconjunto não vazio $K \subseteq \mathbb{R}^{4}$ é convexo se dados $x, y \in K$, então $(1-t) x+t y \in$ $K$ para $0 \leqq t \leqq 1$. Dizemos que uma hipersuperfície $S$, homeomorfa a $S^{3}$, é convexa em $\mathbb{R}^{4}$ se $S$ é o bordo de um subconjunto convexo em $\mathbb{R}^{4}$.

Um $r$-plano suporte de um subconjunto $K \subset \mathbb{R}^{4}$ é uma subvariedade linear de $\mathbb{R}^{4}$ de dimensão $r$, que contém pontos de $\bar{K}$ (o fecho de $K$ ), mas não contém pontos de $\stackrel{\circ}{K}$ (o interior de $K$ ). Um plano suporte é um 3-plano suporte.

Uma hipersuperfície $S, C^{k \geqq 2}$, tem contato de ordem 1 com o hiperplano $H$ em $p \in S$ se para toda curva $C^{2}, \alpha:(-\varepsilon, \varepsilon) \rightarrow S \operatorname{com} \alpha(0)=p$ e $\alpha^{\prime}(0) \neq 0$, então $\left\langle\alpha^{\prime}(0), N_{H}\right\rangle=0$ e $\left\langle\alpha^{\prime \prime}(0), N_{H}\right\rangle \neq 0$ onde $N_{H}$ é um vetor normal a $H$. Um hiperplano $H$ é chamado de plano suporte não singular de $S$ se $H \cap S=\{p\}$, para algum $p \in S$, e $S$ tem contato de ordem 1 com $H$ em $p$. Chamamos uma hipersuperfície $S, C^{k \geqq 2}$, de estritamente convexa se todo plano 
suporte de $S$ é não singular.

Dada uma hipersuperfície regular $S$ estritamente convexa, um ponto $p \in S$ e uma curva $\alpha:(-\varepsilon, \varepsilon) \rightarrow S$ com $\alpha(0)=p$ e $\alpha^{\prime}(0) \neq 0$, temos $\left\langle\alpha^{\prime \prime}(0), N(p)\right\rangle=-\left\langle d N(p) \alpha^{\prime}(0), \alpha^{\prime}(0)\right\rangle \neq 0$. Como isso vale para qualquer $\alpha^{\prime}(0) \in T_{p} S \backslash\{0\}$, então o operador linear $d N(p)$ é definido (ou seja, a forma quadrática associada a $d N(p)$ é definida) e seu sinal depende da orientação de $N(p)$.

Se uma hipersuperficie $S$ for dada na forma $S=H^{-1}(c)$ para alguma função $H: \mathbb{R}^{4} \rightarrow \mathbb{R}$ e $c$ é um valor regular de $H$, então para todo $p \in S$, o vetor normal a $S$ em $p, N(p)$, é dado por $N(p)=\frac{H_{x}(p)}{\left\|H_{x}(p)\right\|}$ onde $H_{x}(p)$ é o vetor gradiente de $H$ no ponto $p$. Desta forma, para $v \in T_{p} S$, temos

$$
\langle d N(p) v, v\rangle=\left\langle\left(\frac{H_{x x}(p)}{\left\|H_{x}(p)\right\|}+\left(s_{i j}(p)\right)\right) v, v\right\rangle
$$

onde $s_{i j}(p)=H_{x_{i}} \frac{\partial \frac{1}{\left\|H_{x}(p)\right\|}}{\partial x_{j}}$. Mas $\left\langle\left(s_{i j}(p)\right) v, v\right\rangle=\left\langle v,\left(s_{i j}(p)\right)^{t} v\right\rangle$ onde $\left(s_{i j}(p)\right)^{t}$ é a matriz transposta a $\left(s_{i j}(p)\right)$ e $\left(s_{i j}(p)\right)^{t} v=0$ para qualquer $v \in T_{p} S$ pois $\left\langle H_{x}(p), v\right\rangle=0$. Concluímos que

$$
\langle d N(p) v, v\rangle=\frac{1}{\left\|H_{x}(p)\right\|}\left\langle H_{x x}(p) v, v\right\rangle
$$

para todo $v \in T_{p} S$. Isso mostra que uma condição necessária para uma hipersuperfície $S=H^{-1}(c)$ ser estritamente convexa é que o Hessiano de $H$ em cada $p \in S$ seja definido quando restrito a $T_{p} S$.

Quando $S$ é difeomorfa a $S^{3}$, então a condição de o Hessiano de $H$ ser definido no espaço tangente de todo ponto de $S$, é suficiente para que a hipersuperfície seja estritamente convexa.

Chamamos de ovalóide uma hipersuperfície regular estritamente convexa e difeomorfa a $S^{3}$.

Dado um ponto $b$ de uma hipersuperfície $S \subset \mathbb{R}^{4}, C^{k \geqq 2}$, com curvatura positiva em $p$, então existe uma vizinhança $C_{b}$ de $b$ em $S$ e um sistema retangular de coordenadas $x_{1}, x_{2}, x_{3}, z$ com $b$ na origem, tal que $C_{b}$ é representada na forma

$$
z=f\left(x_{1}, x_{2}, x_{3}\right)=f(x) \geqq 0, f(0)=0,|x|<\delta, \delta>0
$$

Além disso, $f(x)$ é uma função $C^{k}$ estritamente convexa em $x$, ou seja, o Hessiano de $f$ é positivo definido em todo ponto onde a função $f$ está definida. 


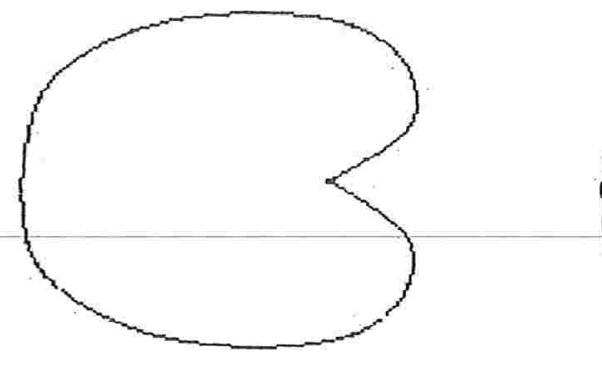

(a)

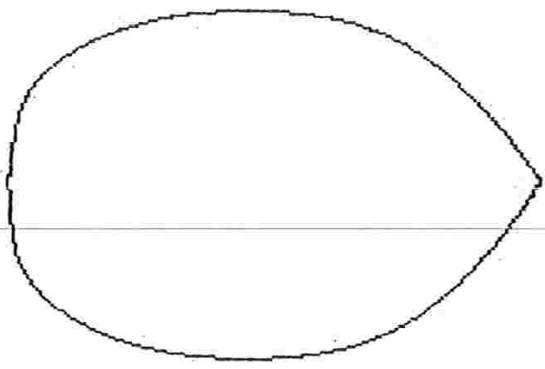

(b)

Figura $^{2}$ 2-1: Uma curva fechada no plano, regular exceto em um ponto e com curvatura positiva pode ser bordo de (a) um domínio não convexo ou (b) um domínio convexo.

\subsection{Regularização de Hipersuperfícies em $\mathbb{R}^{4}$}

Suponhamos que para uma dada função $H: \mathbb{R}^{4} \rightarrow \mathbb{R}, C^{k \geqq 2}$, exista um subconjunto $S \subset H^{-1}(a)$, para algum $a \in \operatorname{Im}(H)$, com as seguintes propriedades:

(i) $S$ é homeomorfo a $S^{3}$;

(ii) existe um único ponto $p_{c} \in S$ tal que $H_{x}\left(p_{c}\right)=0$, ou seja, todo ponto $q \in S, q \neq p_{c}$ é regular;

(iii) para todo ponto $q \in S, q \neq p_{c},\left\langle H_{x x}(q) v, v\right\rangle>0$ para qualquer $v \in T_{q} S$;

(iv) existe um plano suporte $H_{p_{c}}$ de $S$ tal que $H_{p_{c}} \cap S=\left\{p_{c}\right\}$;

O subconjunto $S_{0} \stackrel{\text { def }}{=} S \backslash\left\{p_{c}\right\}$ é, portanto, uma hipersuperfície regular. Vamos mostrar que, de fato, $S_{0}$ é estritamente convexa. Diremos também que $S$ é uma hipersuperfície estritamente convexa.

Se uma hipersuperfície difeomorfa a $S^{3}$ tem curvatura positiva, então ela é estritamente convexa. A presença de um ponto crítico, no entanto, pode causar problemas, como é o caso de uma curva no plano com curvatura positiva e que não é bordo de um domínio convexo. Ver figura 2-1.

Então

Teorema 2.1 Dada qualquer vizinhança $U_{0}$ de $p_{c}$ em $\mathbb{R}^{4}$, existe um ovalóide $S_{U_{0}}$ tal que $S \backslash U_{0} \subset S_{U_{0}}$. 


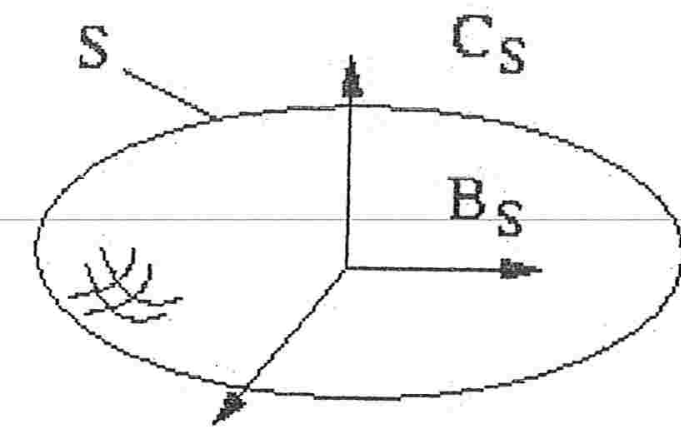

Figura 2-2: Hipersuperfícies em $\mathbb{R}^{4}$ separam o espaço em 2 regiōes: uma limitada $B_{S}$ e outra ilimitada, $C_{S}$.

Antes de provarmos este teorema, precisaremos de alguns lemas.

Sabemos que $S$ separa $\mathbb{R}^{4}$ em dois subconjuntos disjuntos: um limitado, denotado por $B_{S}$, que é fechado e contém $S$, e outro ilimitado, denotado por $C_{S}$, aberto, tal que $\partial B_{S}=\partial C_{S}=S$ e $B_{S} \cap C_{S}=\emptyset$. Ver figura 2-1.

Fixaremos uma orientação $N: S_{0} \rightarrow N S_{0}$ para $S_{0}$ tal que $\left\langle H_{x}(p), N(p)\right\rangle<0$ para todo $p \in S_{0}$. Fixaremos também um referencial móvel sobre $S$ que gera $T S$, ou seja, campos de vetores $X_{i}: S \rightarrow T S, i=1 . .3$, ortonormais. A existência desse referencial será apresentada em (4.2).

Lema 2.1 Seja $p \in S$ um ponto regular. Então existe uma vizinhança $C_{p}$ de $p$ em $S$ e um. sistema retangular de coordenadas $\left(x_{1}, x_{2}, x_{3}, x_{4}\right)$ tal que $C_{p}$ pode ser representada como gráfico de uma função $f^{p}: W \subset \mathbb{R}^{3} \rightarrow \mathbb{R}, x_{4}=f^{p}\left(x_{1}, x_{2}, x_{3}\right)$ onde $f^{p}(0)=0, f_{x}^{p}(0)=0, f^{p}(x)>0$ se $x \neq 0$ e o Hessiano $f_{x x}^{p}(x)$ de $f^{p}$ é positivo definido para todo $x \in W$.

Prova. Os vetores $X_{1}(p), X_{2}(p), X_{3}(p)$ e $N(p)$ definem uma base ortonormal para $\mathbb{R}^{4}$. Nessa base, temos $z=p+x_{1} X_{1}(p)+x_{2} X_{2}(p)+x_{3} X_{3}(p)+x_{4} N(p)$. Os pontos em $S$ satisfazem $H\left(p+x_{1} X_{1}+x_{2} X_{2}+x_{3} X_{3}+x_{4} N\right)=a$ e o ponto $p$ corresponde a $x_{1}=x_{2}=x_{3}=x_{4}=0$. Como $\left\langle H_{x}(p), N(p)\right\rangle \neq 0$, existe $f^{p}: W \subset \mathbb{R}^{3} \rightarrow \mathbb{R}$ numa vizinhança $W$ da origem tal que $x_{4}=f^{p}\left(x_{1}, x_{2}, x_{3}\right)$ e $H\left(p+x_{1} X_{2}+x_{2} X_{2}+x_{3} X_{3}+f^{p}\left(x_{1}, x_{2}, x_{3}\right) N\right)=a$. Portanto o gráfico de $f^{p}$ representa $S$ numa vizinhança de $p$. Além disso, para $i=1,2$ e 3 temos

$$
\left\langle H_{x}(p), X_{i}(p)+f_{x_{i}}^{p}(0) N(p)\right\rangle=0 \Longrightarrow f_{x_{i}}^{p}(0)=0
$$




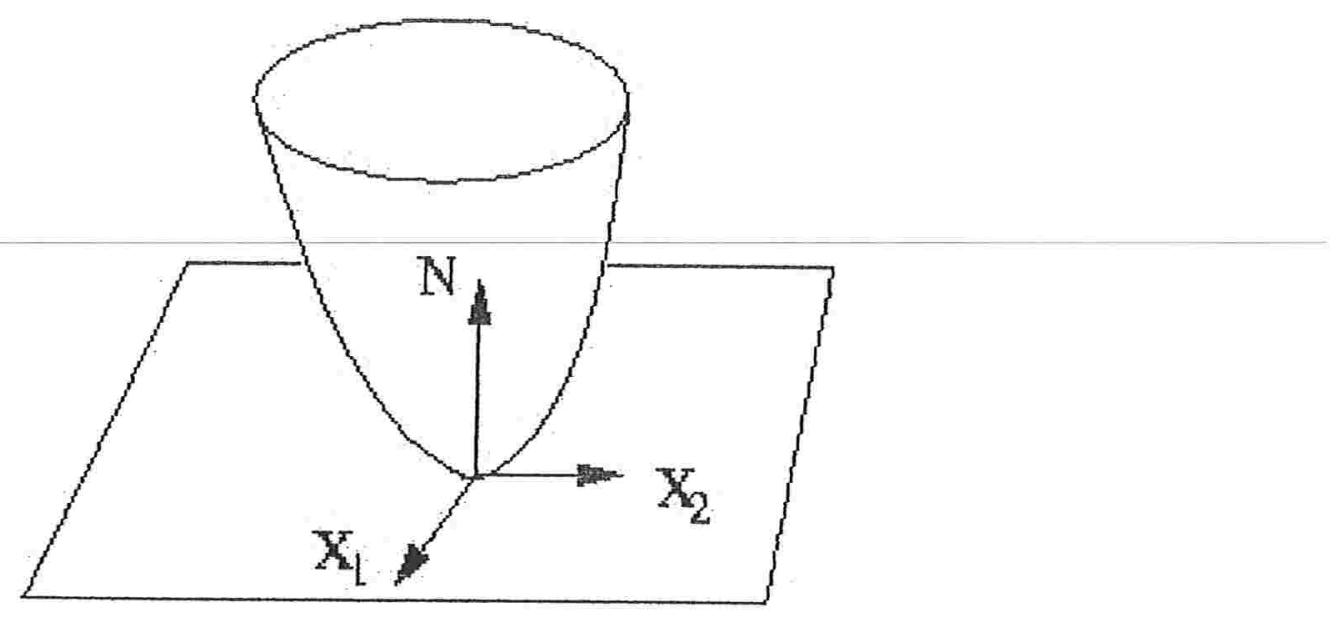

Figura 2-3: A representação local $f^{p}$ de $S$ numa vizinhança de um ponto com cur. vatura positiva é estritamente convexa numa vizinhança da origem.

Também, é fácil ver que

$$
f_{x_{i} x_{j}}^{p}(0)=-\frac{\left\langle H_{x x}(p) X_{i}, X_{j}\right\rangle}{\left\langle H_{x}(p), N\right\rangle}, 1 \leqq i, j \leqq 3
$$

e, portanto, o Hessiano de $f^{p}$ é positivo definido numa vizinhança $W$ de $p$. Logo $f^{p}>0$ em $W$.

Definindo a aplicação $O_{p}: \bar{W} \subset \mathbb{R}^{4} \rightarrow \mathbb{R}^{4}$, numa vizinhança $\bar{W}$ da origem, dada por

$$
O_{p}\left(x_{1}, x_{2}, x_{3}, x_{4}\right)=p+x_{1} X_{2}(p)+x_{2} X_{2}(p)+x_{3} X_{3}(p)+x_{4} N(p)
$$

temos

$$
\begin{aligned}
& O_{p}\left(\left\{x_{4}=f^{p}\left(x_{1}, x_{2}, x_{3}\right)\right\}\right) \subset S \\
& O_{p}\left(\left\{x_{4} \geqq f^{p}\left(x_{1}, x_{2}, x_{3}\right)\right\}\right) \subset B_{S} \\
& O_{p}\left(\left\{x_{4}<f^{p}\left(x_{1}, x_{2}, x_{3}\right)\right\}\right) \subset C_{S}
\end{aligned}
$$

Isso decorre da conexidade de $S$ e da dependência contínua de $f^{p}$ com relação a $p \in S$. 
Lema 2.2 Seja s um segmento de reta que passa por um ponto $p \in S$ tal que $p$ não seja um dos extremos de s. Então s contém pontos de $C_{S}$.

Prova. Se $p$ é um valor regular então localmente $S$ é representada por um gráfico de um função estritamente covexa $x_{4}=f\left(x_{1}, x_{2}, x_{3}\right)$ tal que 0 representa $p$ e $f(x)>0$ para todo $x \neq p$. O conjunto $C_{S}$ satisfaz $x_{4}<f\left(x_{1}, x_{2}, x_{3}\right)$. Nessas coordenadas, se existe um ponto de $s$ tal que $x_{4}>0$, então, como $s$ passa pela origem, existe um ponto de $s$ tal que $x_{4}<0$. Logo $s \cap C_{S} \neq \emptyset$. Se os pontos de $s$ satisfazem $x_{4}=0$, então para $b=\left(x_{1}, x_{2}, x_{3}, 0\right) \in s$, onde $\left(x_{1}, x_{2}, x_{3}\right) \neq(0,0,0)$ temos que $b \in C_{S}$.

Se $x=p_{c}$, então $s \subset H_{p_{c}}$ (plano suporte de $S$ que passa por $p_{c}$ ) ou $s$ é transversal a $H_{p_{c}}$. Como $H_{p_{c}} \cap S=\left\{p_{c}\right\}$ então $S$ e, portanto $B_{S}$, está contido de forma estrita em um dos lados de $H_{p_{c}}$, ou seja, $B_{S} \cap H_{p_{c}}=\left\{p_{c}\right\}$. Logo, em qualquer uma das situações, $s$ contém pontos de $C_{S}$.

Lema 2.3 $B_{S}$ é um subconjunto convexo de $\mathbb{R}^{4}$ e se $p$ é um ponto regular de $S$, então $H_{p} \cap S=$ $\{p\}$ para todo hiperplano $H_{p}$ tangente a $S$ em $p$.

Prova. Para mostarmos que $B_{S}$ é um subconjunto convexo, basta provarmos que dados $x, y \in S, x \neq y$, o segmento de reta $x y$ que une os pontos $x$ e $y$ está contido em $B_{S}$.

Sejam $x$ e $y$ dois pontos em $S$ tais que $x \neq p_{c}$ e $y \neq p_{c}$. Sabemos que o Hessiano de $H$ em $x$ é definido, o que implica que $S$ tem curvatura positiva em $x$. Podemos, portanto, pelo Lema 2.1, representar $S$ numa vizinhança $C_{x}$ de $x$ como gráfico de $x_{4}=f\left(x_{1}, x_{2}, x_{3}\right)$, onde $f$ é estritamente convexa e satisfaz (2.1). O conjunto $B_{S}$ satisfaz, nessas coordenadas, $x_{4} \geqq$ $f\left(x_{1}, x_{2}, x_{3}\right)$. Para $w \in C_{x}$, o segmento $x w$ está, portanto, contido em $B_{S}$.

Seja $\gamma_{x y}:[0,1] \rightarrow S$ uma curva contínua em $S$ tal que $\gamma_{x y}(0)=x, \gamma_{x y}(1)=y$ e $\gamma_{x y}(t) \neq p_{c}$ para todo $t \in(0,1)$. Para $t$ suficientemente pequeno, sabemos, pela observação acima, que o segmento $x \gamma_{x y}(t) \subset B_{S}$ e é transversal a $S$ em $x$ e em $\gamma_{x y}(t)$. Seja $F=\left\{t \in(0,1) \mid x \gamma_{x y}(t)\right.$ $\left.\varsubsetneqq B_{S}\right\}$. Suponhamos que $F \neq \emptyset$ e seja $\tilde{t}=\inf F>0$. Então temos

(i) $x \gamma_{x y}(\tilde{t}) \cap C_{S}=\emptyset$ pois, caso contrário, por continuidade de $x \gamma_{x y}(t)$ e abertura de $C_{S}$, $x \gamma_{x y}(t) \cap C_{S} \neq \emptyset$ para $t<\tilde{t}$, uma contradição. Portanto $x \gamma_{x y}(\tilde{t}) \subset B_{S}$.

(ii) O segmento $x \gamma_{x y}(\tilde{t})$ é transversal a $S$ em $x$ e em $\gamma_{x y}(\tilde{t})$, pois se for tangente em algum desses pontos, existem pontos de $x \gamma_{x y}(\tilde{t})$ em $C_{S}$, uma contradição por (i). Suponha 
que $x \gamma_{x y}(\tilde{t}) \cap S=\left\{x, \gamma_{x y}(\tilde{t})\right\}$. Para todo $t$ suficientemente próximo de $\tilde{t}, x \gamma_{x y}(t)$ também é transversal em $x$ e em $\gamma_{x y}(t)$ e, por continuidade de $\gamma_{x y}(t)$, tem-se que $x \gamma_{x y}(t) \subset B_{S}$, o que contradiz a hipótese sobre $\tilde{t}$.

(iii) Portanto existe $w \in x \gamma_{x y}(\hat{t}), w \notin\left\{x, \gamma_{x y}(\tilde{t})\right\}$ tal que $w \in S$. Mas pelo Lema 2.2, o segmento de reta $x \gamma_{x y}(\tilde{t})$ contém pontos de $C_{S}$, uma contradição com (i).

Concluímos que $F=\emptyset \mathrm{e}$, por continuidade de $x \gamma_{x y}(t)$ e compacidade de $B_{S}$ temos que o segmento $x y \subset B_{S}$.

Se $y=p_{c}$, então seja $\gamma_{x p}:[0,1] \rightarrow S$ uma curva contínua em $S$ tal que $\gamma_{x y}(0)=x$, $\gamma_{x y}(1)=p_{c}$ e $\gamma_{x y}(t) \neq p_{c}$ para todo $t \in(0,1)$. Então o segmento $x \gamma_{x y}(t) \in B_{S}$ para todo $t \in(0,1)$ e, novamente por continuidade de $x \gamma_{x y}(t)$ e compacidade de $B_{S}$ concluímos que o segmento $x p \subset B_{S}$.

Portanto para quaisquer $x, y \in B_{S}$ o segmento $x y$ está contido em $B_{S}$ e, portanto, $B_{S}$ é um subconjunto convexo de $\mathbb{R}^{4}$.

Suponha que exista $p \in S$ e um hiperplano $H_{p}$ de $B_{S}$ passando por $p$ tal que $H_{p} \cap S \supset\{p, z\}$ onde $z \in B_{S}, z \neq p$. Como $B_{S}$ é convexo, o segmento $p z \subset B_{S}$ e portanto $H_{p} \cap B_{S} \supset p z$. Pelo Lema 2.2 sabemos $p z \cap S=\{p, z\}$ pois caso contrário o segmento $p z$ teria pontos em $C_{S}$. Mas então $H_{p}$ contém pontos de $\stackrel{\circ}{B_{S}}$ (o interior de $B_{S}$ ) o que contraria a hipótese de $H_{p}$ ser um plano suporte de $B_{S}$.

Corolário 2.1 A hipersuperfície $S_{0}=S \backslash\left\{p_{c}\right\}$ é estritamente convexa.

Prova. Sabemos, pela definição de $S$, que $S_{0}$ tem contato de ordem $1 \mathrm{com}$ todo hiperplano $H_{p}$ tangente a $S$ em $p$. Pelo Lema 2.3 temos que $H_{p} \cap S_{0}=\{p\}$ e, portanto, $H_{p}$ é um plano suporte não singular de $S$, donde $S_{0}$ é estritamente convexa.

Antes de enunciarmos o resultado principal desta seção, precisamos do seguinte teorema de M. Ghomi

Teorema 2.2 Seja $S$ uma hipersuperfície $C^{2}$ compacta, conexa e imersa em $\mathbb{R}^{4}$ com curvatura positiva. Então $S$ pode ser estendida a um ovalóide $C^{k}$ se e somente se cada componente $\gamma$ do bordo de $S$ se localiza de forma estrita em um dos lados de cada hiperplano tangente a $S$ em $\gamma$.

Prova. Ver [Gho2]. 


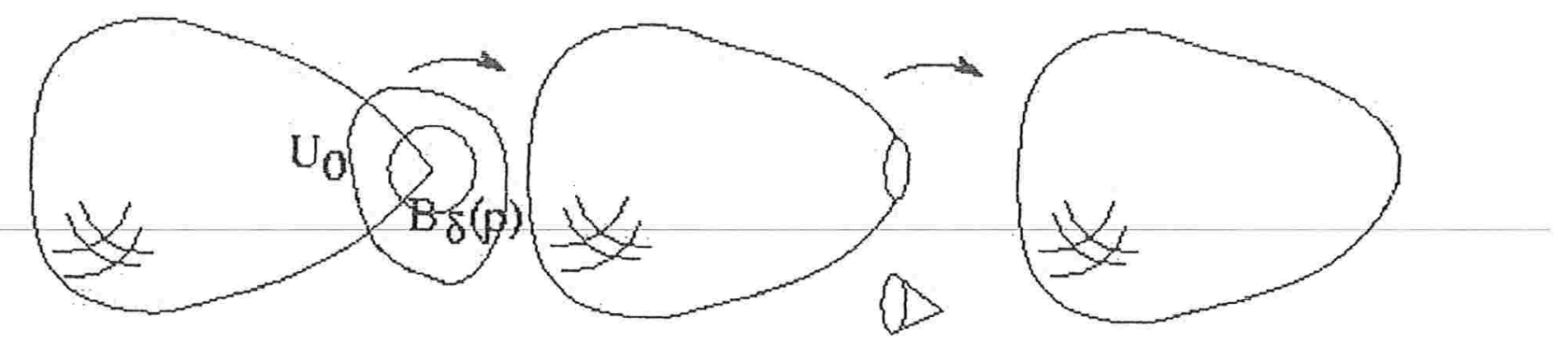

Figura 2-4: O Teorema de Ghomi permite regularizar uma hipersuperficie na vizinhança do ponto crítico.

Prova do Teorema 2.1.Seja $\delta>0$ tal que $B_{\delta}(p) \subset U_{0}$ onde $B_{\delta}(p)$ é a bola de raio $\delta$ conn centro em $p$. Seja $S_{\delta}=S \backslash B_{\delta}(p)$. Seja $\partial S_{\delta}$ o bordo de $S_{\delta}$. Então $\partial S_{\delta}=\partial B_{\delta}(p) \cap S$. Seja $z \in$ $\partial S_{\delta}$. Então o hiperplano $H_{z}$ tangente a $S_{\delta}$ em $z$ é o mesmo hiperplano tangente a $S$ em $z$. Pelo Lema 2.3 sabemos que $H_{z} \cap S=\{z\}$. Logo $H_{z} \cap S_{\delta}=\{z\}$. Como isso vale para qualquer $z \in \partial S_{\delta}$, podemos, pelo Teorema 2.2, estender $S_{\delta}$ a um ovalóide $S_{U_{0}}$. Como $B_{\delta}(p) \subset U_{0}$ e $S_{U_{0}} \supset S_{\delta}=S \backslash B_{\delta}(p)$ então $S_{U_{0}} \supset S \backslash U_{0}$. Ver figura 2.4 


\section{Capítulo 3}

\section{Pontos de equilíbrio do tipo sela-centro}

Um ponto $p \in \mathbb{R}^{4}$ é chamado ponto de equilíbrio do sistema Hamiltoniano associado à função $H: \mathbb{R}^{4} \rightarrow \mathbb{R}$ se $H_{x}(p)=0$, ou seja, se a solução que passa por $p$ for constante ${ }^{1}$. Dizemos que um ponto de equilíbrio $p$ é do tipo sela-centro se a matriz $J_{0} H_{x x}(p)$ possui um par de autovalores reais $\pm \alpha(\alpha>0)$ e um par de autovalores imaginários puros $\pm \omega i(\omega>0)$.

Um ponto de equilíbrio $p$ do tipo sela-centro possui variedades estável e instável unidimensionais. Uma solução não-constante é chamada de órbita homoclínica a $p$, caso esteja contida em ambas as variedades estável e instável de $p$. No caso de um sela-centro, a existência de tal órbita homoclínica implica que as variedades assintóticas a $p$ devem coincidir.

Numa vizinhança de um sela-centro $p$, existe uma família contínua de órbitas periódicas, uma para cada valor de $H$, constituindo a variedade central bi-dimensional de $p$. Da mesma forma se define órbita homoclínica a tais órbitas periódicas. Neste caso, a existência de uma órbita homoclínica a alguma órbita periódica $\gamma$ desta família não implica que as variedades estável e instável de $\gamma$ devam coincidir, já que estas são bi-dimensionais.

Por um Teorema de Moser [Mo] (veja também [Russ] e [Hen]), sabemos que numa vizinhança $U$ de um ponto de equilíbrio $p$ do tipo sela-centro, o fluxo possui uma forma normal bastante

\footnotetext{
${ }^{1}$ As equações de um sistema Hamiltoniano associado à função $H$ são dadas por $\dot{x}=J_{0} H_{x}(x)$, onde $J_{0}=$ $\left(\begin{array}{ll}0 & I_{2 \times 2} \\ -I_{2 \times 2} & 0\end{array}\right)$ e $H_{x}(x)$ é o gradiente de $H$ no ponto $x$.
} 
simples. Em outras palavras, podemos encontrar uma mudança de coordenadas $\varphi: 0 \in V \subset$ $\mathbb{R}^{4} \rightarrow U$ onde, se denotarmos por $z=\left(q_{1}, q_{2}, p_{1}, p_{2}\right)$ as coordenadas em $V$, então o fluxo em $U$ é conjugado ao fluxo Hamiltoniano em $V$ gerado pela função Hamiltoniana

$$
K\left(q_{1}, q_{2}, p_{1}, p_{2}\right)=-\alpha I_{1}+\omega I_{2}+O\left(I_{1}^{2}+I_{2}^{2}\right)
$$

onde $I_{1}=q_{1} p_{1}$ e $I_{2}=\frac{q_{2}^{2}+p_{2}^{2}}{2}$. As constantes $\alpha$ e $\omega$ são aquelas obtidas pelos autovalores do sistema linearizado em $p$. As expressões $I_{1}$ e $I_{2}$ são integrais primeiras do sistema, ou seja, permanecem constantes ao longo de cada solução. Em $V$, as equações que determinam o movimento são

$$
\begin{aligned}
& \dot{q}_{1}=\frac{\partial K\left(I_{1}, I_{2}\right)}{\partial I_{1}} q_{1} \\
& \dot{p}_{1}=-\frac{\partial K\left(I_{1}, I_{2}\right)}{\partial I_{1}} p_{1} \\
& \dot{q}_{2}=\frac{\partial K\left(I_{1}, I_{2}\right)}{\partial I_{2}} p_{2} \\
& \dot{p}_{2}=-\frac{\partial K\left(I_{1}, I_{2}\right)}{\partial I_{2}} q_{2}
\end{aligned}
$$

Esse sistema pode ser melhor visualizado em dois subespaços distintos: o plano $\left(q_{1}, p_{1}\right)$, onde o comportamento é o de uma sela hiperbólica, e o plano $\left(q_{2}, p_{2}\right)$ onde o sistema se comporta como um centro. Ver figura 3-1

Algumas propriedades do fluxo em $U$ podem, portanto, ser obtidas pela análise do fluxo em $V:$

i) $0 \in V$ é um ponto de equilíbrio do tipo sela-centro e $\varphi(0)=p$;

ii) Os conjuntos $W^{s}(0) \stackrel{\text { def }}{=}\left\{z \in V: p_{1}=p_{2}=q_{2}=0\right\}$ e $W^{u}(0) \stackrel{\text { def }}{=}\left\{z \in V: q_{1}=p_{2}=q_{2}=\right.$ $0\}$ são, respectivamente, as variedades estável e instável de $0 \mathrm{em} V$; Ambas estão contidas em $\{K=0\}$, o mesmo nível de energia da origem;

iii) As órbitas periódicas $P_{E}$, que compõe a variedade central de 0 estão contidas em $\{z \in$ $\left.V: q_{1}=p_{1}=0, q_{2}^{2}+p_{2}^{2}>0\right\}$. Para cada valor de energia $k \subset K(V), k \neq 0$, existe uma única órbita periódica $o_{k}$ com energia $k$ contida na variedade central do sela-centro. As variedades estável e instável de uma órbita peródica com energia $k$ estão contidas nos conjuntos $W^{s}\left(o_{k}\right) \stackrel{\text { def }}{=}$ $\left\{z \in V: p_{1}=0, K=k\right\}$ e $W^{u}\left(o_{k}\right) \stackrel{\text { def }}{=}\left\{z \in V: q_{1}=0, K=k\right\}$ respectivamente. 

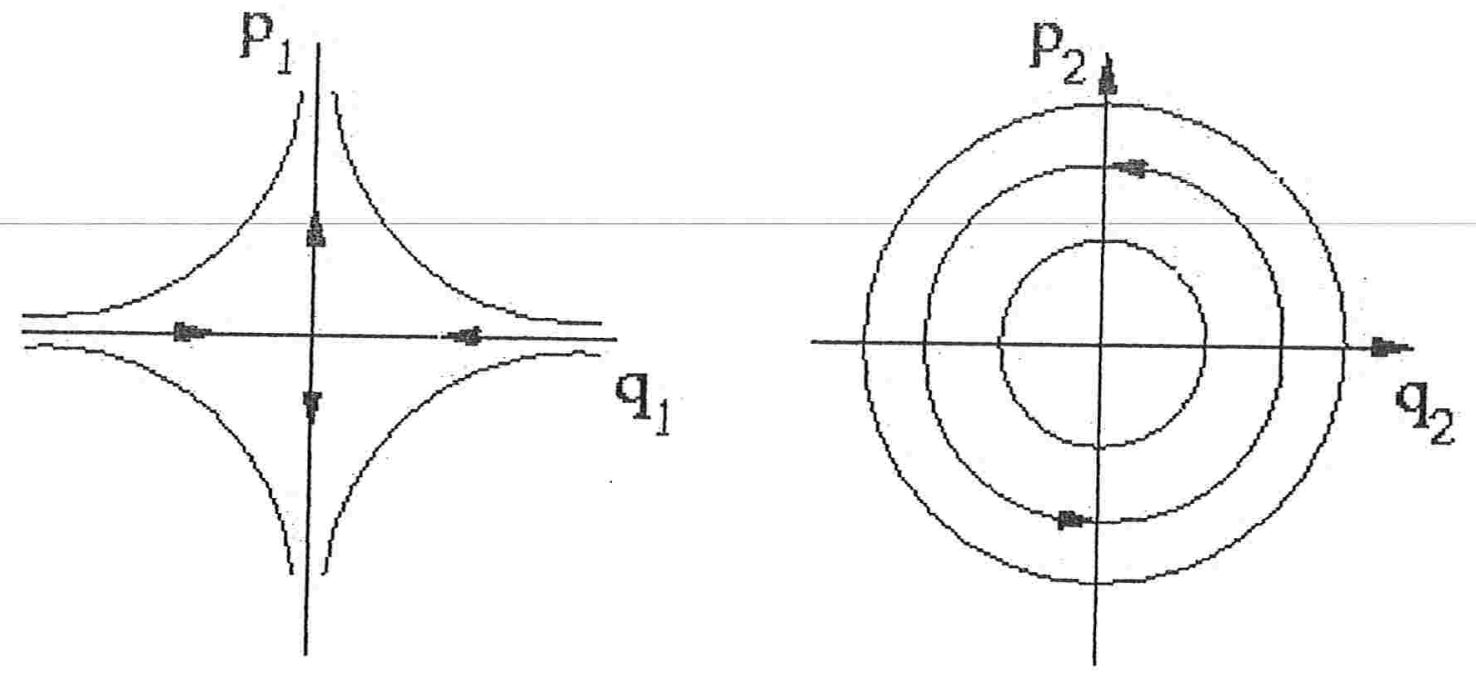

Figura 3-1: Na forma normal de um sela centro, a projeção do fluxo no plano $q_{1} p_{1}$ é equivalente a uma sela e no plano $q_{2} p_{2}$ é equivalente a um centro.

O uso de seções de Poincaré é uma ferramenta importante para descrevermos o fluxo numa vizinhança de um sela-centro. Em particular, os conjuntos $\sum_{1}=\left\{z \in V: q_{1}=\delta,\right\}$ e $\sum_{2}=$ $\left\{z \in V: p_{1}=\delta,\right\}$ definem seções transversais ao fluxo em $V$, e intersectam transversalmente, respectivamente, as variedades estável e instável da origem e das órbitas periódicas que folheam sua variedade central.

Vamos denotar por $\sum_{i}^{E}$ a intersecção de $\sum_{i}$ com o nível de energia $\{K=E\}, i=1,2$. Por (3.1) vemos que $\frac{\partial K}{\partial I_{1}}(0,0)=-\alpha \neq 0$. Pelo Teorema da Função Implícita, obtemos $I_{1}=v\left(I_{2}, E\right)$ numa vizinhança de $I_{2}=0 \mathrm{e}$, portanto, podemos tomar $\left(q_{2}, p_{2}\right)$ como coordenadas em $\sum_{i}^{E}$. Nessas coordenadas, denotando $y=\left(q_{2}, p_{2}\right)$, obtemos a aplicação de Poincaré $l_{E}: \sum_{1}^{E} \cap\{p 1>$ $0\} \rightarrow \sum_{2}^{E}$ dada por

$$
l_{E}(y)=R\left(\theta\left(I_{2}(y), E\right)\right) y
$$

onde $R(\theta)$ denota a matriz de rotação de ângulo $\theta$, e.

$$
\theta\left(I_{2}, E\right)=-\frac{\omega}{\alpha} \log \left|I_{2}-I^{c}(E)\right|+\Lambda_{E}\left(I_{2}\right)
$$

onde $I^{c}(E)=\frac{E}{\omega}+O\left(E^{2}\right)$ é tal que $v\left(I^{c}(E), E\right)=0$. A função $\Lambda_{E}$ é analítica em $I_{2}$ e os pontos em $\sum_{1}^{E}$ satisfazem $I_{2}>I^{c}(E)$. As relações (3.2) e (3.3) mostram que localmente o fluxo, 
em cada nível de energia $E$, pode ser visto como uma rotação cujo ângulo de rotação tende para $+\infty$ conforme a órbita se aproxima das variedades estável e instável do conjunto invariante em $V$ contido no mesmo nível de energia $E$. Por exemplo, para $E=0$, o conjunto invariante em $V$ é o próprio ponto de equilíbrio sela-centro e a aplicação $l_{0}$ está definida apenas para $y \neq 0$. O ângulo de rotação depende apenas de $|y|$ e tende para $+\infty$ quando $y \rightarrow 0$. Quando $E>0$ o conjunto invariante em $V$ é uma órbita periódica $o_{E}$ e a aplicação $l_{E}$ está definida apenas para $|y|^{2}>2 I^{c}(E)$. O ângulo de rotação, nesse caso, tende para $+\infty$ quando $y$ se aproxima de círculo $|y|^{2}=2 I^{c}(E)$. É importante notar que para $E=0$, a aplicação $l_{0}$ pode ser estendida de forma contínua na origem definindo-se $l_{0}(0) \stackrel{\text { def }}{=} 0$.

Quando existe uma órbita homoclínica à $p$, sabemos, usando o Teorema do Fluxo Tubular, que existe uma aplicação analítica $g_{E}: 0 \in W \subset \sum_{2}^{E} \rightarrow \sum_{1}^{E}$ satisfazendo $g_{0}(0)=0$.

Compondo as aplicações $l_{E}$ e $g_{E}$, podemos estudar a aplicação $F_{E} \stackrel{\text { def }}{=} g_{E} \circ l_{E}$ que representa a dinâmica próxima à órbita homoclínica a $p$.

\subsection{Perturbações de um Sistema Integrável}

Seja $H_{\mu}$ uma família a um parâmetro $\mu \in I_{0}=(-\bar{\mu}, \bar{\mu})$ de funções Hamiltonianas real-analíticas definidas em $\mathbb{R}^{4}$ que possuem as seguintes propriedades:

i) Рага $\mu=0$ o campo de vetores associado a $H_{0}$ é integrável e existe uma órbita homoclínica $\gamma$ à origem;

ii) Para cada valor de $\mu \in I_{0}$, existe um equilíbrio $r_{\mu}$ do tipo sela-centro do campo de vetores associado a $H_{\mu}$ que depende analiticamente em $\mu$;

Sem perda de generalidade, podemos assumir também $H\left(r_{\mu}\right)=0$.

Utilizando as mesmas notações anteriores mas agora acrescentando o índice $\mu$, obtemos os mapas $l_{E, \mu}$ e $g_{E, \mu}$ que correspondem às aplicações local e global do fluxo, respectivamente para cada par $(E, \mu)$. Considerando a aplicação $F_{E, \mu}=g_{E, \mu} \circ l_{E, \mu}$, temos que $F_{E, 0}=R\left(\zeta\left(I_{2}, E\right)+\right.$ $\left.\theta\left(I_{2}, E\right)\right)$, pois o sistema é integrável para $\mu=0$.

O Teorema KAM (ver [BGS] e [Rag2]) garante que para valores de $\mu$ e $E$ suficientemente pequenos, existem curvas $C_{E, \mu}$ com as seguintes propriedades:

i) $C_{E, \mu} \subset \operatorname{dom} F_{E, \mu}$; 


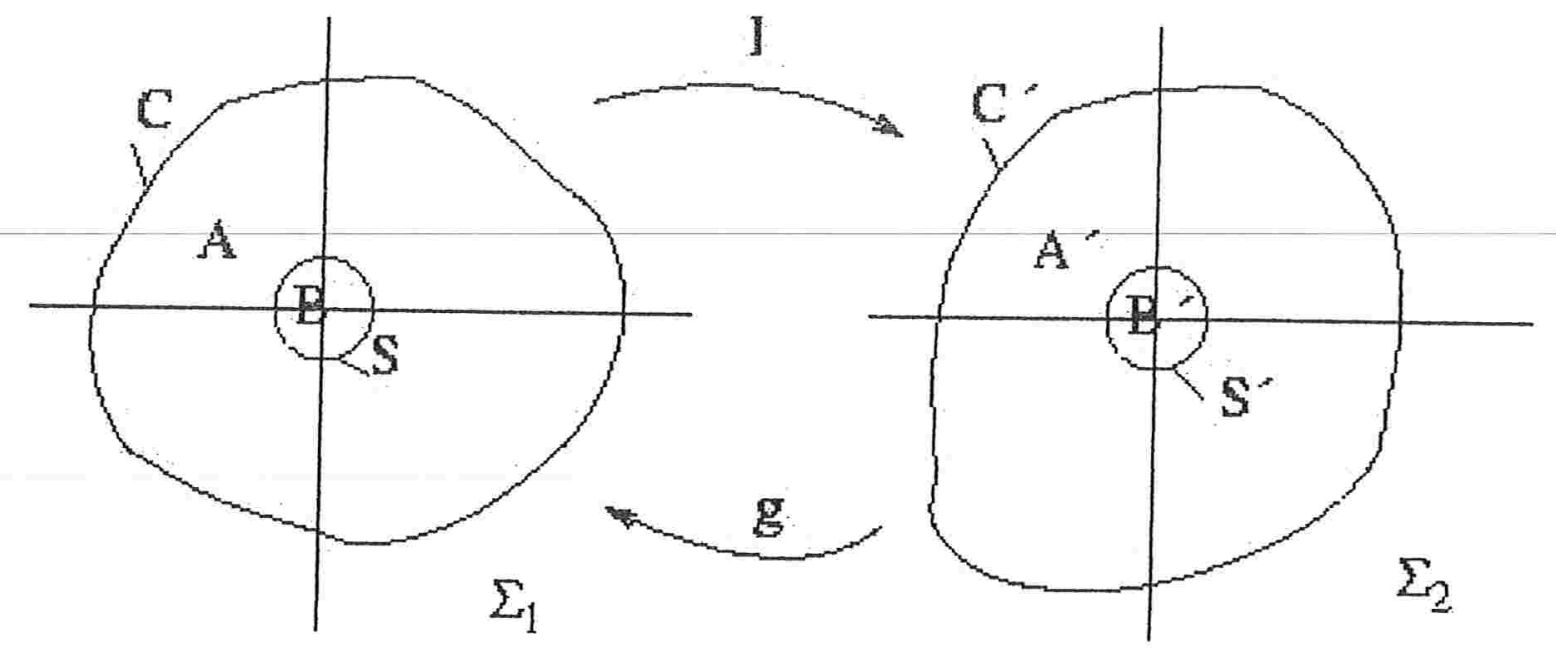

Figura $\sim 2$ :

ii) $C_{E, \mu}$ são curvas analíticas de Jordan que enlaçam o círculo $I_{2}=I^{c}(E)$;

iii) $C_{E, \mu}$ são invariantes por $F_{E, \mu}$;

Seja $C_{E, \mu}^{\prime}=l_{E, \mu}\left(C_{E, \mu}\right)=g_{E, \mu}^{-1}\left(C_{E, \mu}\right)$.

\section{2 Órbitas Homoclínicas à variedade central de um sela-centro}

Por simplicidade de notação, omitiremos os índice $E$ e $\mu$. Analisaremos a partir de agora apenas os níveis de energia positivos.

Denotaremos por $S\left(S^{\prime}\right)$ a intersecção entre a variedade estável (instável) e $\sum_{1}\left(\sum_{2}\right)$. Seja $A\left(A^{\prime}\right)$ o anel aberto entre $S\left(S^{\prime}\right)$ e $C\left(C^{\prime}\right)$. Seja $B\left(B^{\prime}\right)$ o disco fechado limitado por $S\left(S^{\prime}\right)$, e $D\left(D^{\prime}\right)$ o disco aberto limitado por $C\left(C^{\prime}\right)$.

Então temos as seguintes aplicações: $l: A \rightarrow A^{\prime}$ e $g: D^{\prime} \rightarrow D$. Ambos as aplicações preservam área. Ver figura 3-2

Suponhamos que $w \in g\left(S^{\prime}\right) \cap S \neq \varnothing$. Então existe uma órbita $h_{P}$ que passa por $w$ e é homoclínica à órbita periódica $P$. Chamamos $h_{P}$ de órbita homoclínica a $P$ de 1 pulso. Mais geralmente se para algum $w \in S^{\prime}$ tivermos $F^{N-1} \circ g(w) \in S$, então temos uma órbita homoclínica a $P$ de $N$ pulsos. Provaremos que para sistemas próximos ao integrável e níveis de energia suficientemente próximos do nível de energia do sela-centro $r$, sempre há órbitas 
homoclínicas às órbitas periódicas da variedade central de $r$.

Lema 3.1 Suponhamos que para $0 \leqq n \leqq N$, a aplicaçâo $F^{n} \circ g$ esteja bem definido numa vizinhança de $B^{\prime}$ e $F^{n} \circ g\left(S^{\prime}\right) \cap S=\varnothing$. Então $F^{i} \circ g\left(B^{\prime}\right) \cap F^{i} \circ g\left(B^{\prime}\right)=\varnothing$ para todo $0 \leqq j<i \leqq N$.

Prova. Suponhamos que $F^{i} \circ g\left(B^{\prime}\right) \cap F^{j} \circ g\left(B^{\prime}\right) \neq \varnothing$ para $i, j$ tais que $0 \leqq j<i \leqq N$. Por preservação de área e analiticidade das aplicações $l$ e $g$ segue que $F^{i} \circ g\left(S^{\prime}\right) \cap F^{j} \circ g\left(S^{\prime}\right) \neq \varnothing$. Aplicando $F^{-j}$ a ambos os lados, temos que $F^{i-j} \circ g\left(S^{\prime}\right) \cap g\left(S^{\prime}\right) \neq \varnothing$ o que é uma contradição pois a aplicação $F^{-1}$ não está definida em $g\left(S^{\prime}\right)$.

Proposição 3.1 Existe $N \geqq 1$ tal que $F^{N-1} \circ g\left(S^{\prime}\right) \cap S \neq \varnothing$. Além disso, ou $F^{N-1} \circ g\left(S^{\prime}\right)=S$, ou $F^{N-1} \circ g\left(S^{\prime}\right) \varsubsetneqq S$. No segundo caso existem infinitas órbitas homoclínicas de $2 N$ pulsos.

Prova. O Anel $A$ tem área limitada e, pela preservação de área das aplicações $g$ e $l$, temos que $F^{i} \circ g\left(B^{\prime}\right)$ tem sempre a mesma área. A não existência de tal $N$ implica, pelo Lema 3.1 que estes são todos disjuntos, ocupando, portanto, uma área infinita em $A$, uma contradição.

Denotemos $G=F^{N-1} \circ g$ e $\delta=l\left(G\left(S^{\prime}\right) \cap A\right)$. Então se $G\left(S^{\prime}\right) \varsubsetneqq S, \delta$ é uma curva que espirala infinitas vezes convergindo para $S^{\prime}$ de modo que $G(\delta \cap \operatorname{dom} G)$ espirala infinitas vezes convergindo para $G\left(S^{\prime}\right)$ intersectando $S$ infinitas vezes. Essas intersecções representam as órbitas homoclínicas de $2 N$ pulsos.

Pode-se mostrar que nos casos onde $G\left(S^{\prime}\right) \varsubsetneqq S$, existe um conjunto invariante no mesmo nível de energia de $P$ tal que o fluxo restrito a esse conjunto é semi-conjugado ao shift de Bernoulli e tem entropia topológica positiva. Para mais detalhes, ver [BGS].

Vamos agora fixar um valor de parâmetro tal que não exista órbita homoclínica ao ponto de equilíbrio sela-centro, ou seja, tais que suas variedades estável e instável não coincidam. Continuamos considerando o caso próximo ao integrável, ou seja, para todo valor de energia suficientemente pequeno, existem órbitas homoclínicas a $P_{E}$.

Seja $N(E)=\min \left\{n \in \mathbb{N}: F_{E}^{n-1} \circ g\left(S_{E}^{\prime}\right) \cap S_{E} \neq \varnothing\right\}$

Teorema 3.1 Existe uma seqüência $E_{n} \rightarrow 0$ tal que as variedades estável e instável de $P_{E_{n}}$ não coincidem. Além disso $\lim _{E \rightarrow 0} N(E)=\infty$.

Prova. Suponha que exista uma sequiência $E_{n} \rightarrow 0$ tal que $N\left(E_{n}\right)$ seja limitada. Podemos supor que $N\left(E_{n}\right)=N=$ const. Então $F_{E_{n}}^{N-1} \circ g\left(S_{E_{n}}^{\prime}\right) \cap S_{E_{n}} \neq \varnothing$. Usando um argumento 
de compacidade e continuidade das aplicações, concluimos que $F_{0}^{N-1} \circ g(0)=0$, o que é uma contradição, já que não há órbita homoclínica à origem.

Se não existir uma seqüência $E_{n} \rightarrow 0$ tal que as variedades estável e instável de $P_{E_{n}}$ não coincidam, então para qualquer $E$ suficientemente pequeno as variedades assintóticas devem coincidir. Mas um mesmo argumento de compacidade e continuidade implica que para $E$ suficientemente pequeno, $N(E)$ é constante, uma contradição. 


\section{Capítulo 4}

\section{O Índice de Conley-Zehnder}

\subsection{Uma Trivialização natural de fibrados lineares sobre Hiper- superficies em $\mathbb{R}^{4}$}

Seja $H: \mathbb{R}^{4} \rightarrow \mathbb{R}$ uma função $C^{\infty}$ e $c$ um valor regular de $H$. Seja $F_{S}$ um fibrado linear sobre a hipersuperfície $S \stackrel{\text { def }}{=} H^{-1}(c), C^{k \geqq 2}$, tal que a fibra $\xi_{x}$ em cada $x \in S$ é um subespaço de $T_{x} S$ de dimensão $n(n=1,2$ ou 3$)$. Então o fibrado $F_{S}$ pode ser trivializado se existir um homeomorfismo $\beta_{F_{S}}: F_{S} \rightarrow S \times \mathbb{R}^{n}$ tal que $\operatorname{pr} 1 \circ \beta_{F_{S}}=\pi_{F_{S}}$ onde $\operatorname{pr} 1$ é a projeção na primeira componente de $S \times \mathbb{R}^{n}$ e $\pi_{F_{S}}$ é a projeção do fibrado $F_{S}$ sobre a base $S$. O homeomorfismo $\beta_{F_{S}}$ é chamado de trivialização de $F_{S}$. Introduziremos uma forma natural de trivializar o fibrado tangente $T S$ apresentada em [CPR]. Usaremos esse mesmo método para trivializar fibrados cuja fibra bi-dimensional é transversal ao campo Hamiltoniano associado à função $H$.

$$
\text { Sejam } I=\left(\begin{array}{ll}
1 & 0 \\
0 & 1
\end{array}\right), J=\left(\begin{array}{cc}
0 & 1 \\
-1 & 0
\end{array}\right) \text { e } 0=\left(\begin{array}{ll}
0 & 0 \\
0 & 0
\end{array}\right) \text {. Definimos agora as matrizes } 4 \times 4
$$

dadas por:

$$
A_{0}=\left(\begin{array}{ll}
I & 0 \\
0 & I
\end{array}\right), A_{1}=\left(\begin{array}{ll}
0 & J \\
J & 0
\end{array}\right), A_{2}=\left(\begin{array}{cc}
J & 0 \\
0 & -J
\end{array}\right) \text { e } A_{3}=\left(\begin{array}{cc}
0 & I \\
-I & 0
\end{array}\right)
$$

Para cada $x \in S$, seja

$$
X_{0}(x) \stackrel{\text { def }}{=} \frac{H_{x}(x)}{\left\|H_{x}(x)\right\|}
$$


onde $H_{x}(x) \in T_{x} \mathbb{R}^{4} \simeq \mathbb{R}^{4}$ é o vetor gradiente de $H$ no ponto $x$, é é normal a $T_{x} S$. Sejam

$$
X_{i}(x) \stackrel{\text { def }}{=} A_{i} X_{0}(x), i=1,2,3
$$

Temos que $\left\langle X_{i}(x), X_{j}(x)\right\rangle=0$ para $0 \leqq i \neq j \leqq 3$ e $\left\langle X_{i}(x), X_{i}(x)\right\rangle=1$ para todo $i \epsilon$ $\{0,1,2,3\}$. Portanto, os vetores $X_{1}(x), X_{2}(x)$ e $X_{3}(x)$ formam uma base ortonormal para $T_{x} S$. Como $X_{i}(x) \neq 0$ para qualquer $x \in S$, podemos trivializar $T S$ usando os campos $X_{1}, X_{2}$ e $X_{3}$ da seguinte forma: todo vetor $v$ tangente a $S$ em $x$ pode ser representado por $v=\sum_{i=1}^{3} \alpha^{i} X_{i}(x)$. Então definimos a trivialização $\beta_{T S}: T S \rightarrow S \times \mathbb{R}^{3}$ por $\beta_{T S}(x, v)=\left(x, \alpha^{1}, \alpha^{2}, \alpha^{3}\right)^{i=}$.

Sabemos que o campo Hamiltoniano associado à função $H$ é dado por $X_{H}(x)=A_{3} H_{x}(x)$ e, portanto, $X_{H}(x)=\delta(x) X_{3}(x)$ onde $\delta(x)=\left\|H_{x}(x)\right\|$. Desta forma, podemos considerar o fibrado linear $F_{2}$ sobre $S$ cuja fibra é gerada pelos campos $X_{1}$ e $X_{2}$. O fibrado $F_{2}$ é bidimensional, normal a $X_{H}(x)$ e pode ser trivializado da mesma forma como foi trivializado TS.

Consideremos agora um outro fibrado $\xi$ sobre $S$, bi-dimensional, contido em $T S$, cuja fibra $\xi_{x}$ é transversal a $X_{3}(x)$ para todo ponto $x \in S$.

Teorema 4.1 O fibrado $\xi$ pode ser trivializado.

Prova. Como $\xi_{x}$ é transversal a $X_{3}(x)$, então para cada vetor não nulo $v \in \xi_{x}$, temos que $v=\alpha^{1} X_{1}(x)+\alpha^{2} X_{2}(x)+\alpha^{3} X_{3}(x)$ onde $\left(\alpha^{1}\right)^{2}+\left(\alpha^{2}\right)^{2} \neq 0$.

Seja $\pi_{\xi_{x}}: \xi_{x} \rightarrow\left\{X_{1}, X_{2}\right\}$ a projeção canônica dada por $\pi_{\xi_{x}}(v)=\alpha^{1} X_{1}(x)+\alpha^{2} X_{2}(x)$. Então $\pi_{\xi_{x}}$ é um isomorfismo e podemos, portanto, definir uma base em $\xi_{x}$ dada por $\left\{\pi_{\xi_{x}}^{-1}\left(X_{1}(x)\right), \pi_{\xi_{x}}^{-1}\left(X_{2}(x)\right)\right\}$. Definindo

$$
\tilde{X}_{i}(x)=\pi_{\xi_{x}}^{-1}\left(X_{i}(x)\right), i=1,2
$$

temos

$$
v=\alpha^{1} \tilde{X}_{1}(x)+\alpha^{2} \tilde{X}_{2}(x)
$$

de modo que os campos $\left\{\widetilde{X}_{1}, \widetilde{X}_{2}\right\}$ induzem uma trivialização $\beta_{\xi}: \xi \rightarrow S \times \mathbb{R}^{2}$ para $\xi$, dada por

$$
\beta_{\xi}(x, v)=\left(x, \alpha^{1}, \alpha^{2}\right)
$$




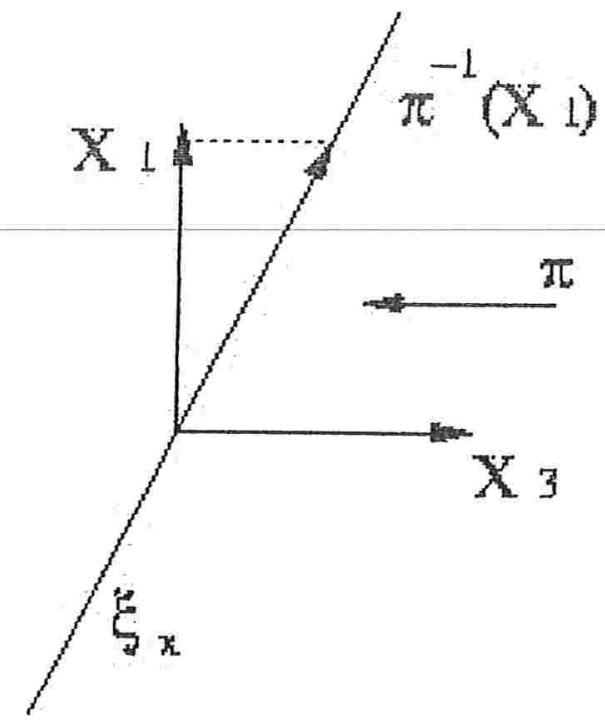

Figura 4-1: Os vetores $\tilde{X}_{1}$ e $\tilde{X}_{2}$ definem uma base para $\xi_{x}$.

Ver figura 4-1.

Observe que as coordenadas na trivialização $\beta_{\xi}$ são iguais às 2 primeiras coordenadas na trivialização $\beta_{T S}$.

Seja $\varphi_{t}$ o fluxo do sistema Hamiltoniano associado à função $H$, ou seja, $\frac{d \varphi_{t}(x)}{d t}=X_{H}(x)$. Linearizando as equações de movimento sobre uma solução $x(t) \subset S$, obtemos um fluxo $T \varphi_{t}$ : $T S \rightarrow T S$ que satisfaz a seguinte equação

$$
\dot{y}=A_{3} H_{x x}(x(t)) y
$$

onde $H_{x x}(x)$ é a matriz Hessiana de $H$ no ponto $x$ e $y(t) \in T_{x(t)} S$. No referencial que trivializa $T S$, temos $y(t)=\sum_{i=1}^{3} \alpha^{i}(t) X_{i}(x(t))$. Denotando $\alpha=\left(\begin{array}{c}\alpha^{1} \\ \alpha^{2}\end{array}\right)$ obtemos de (4.5)

$$
\dot{\alpha}=-J \stackrel{\sim}{S} \alpha
$$


onde $\tilde{S}$ é a matriz dada por

$$
\tilde{S}=\left(\begin{array}{cc}
\left\langle H_{x x} X_{1}, X_{1}\right\rangle & \left\langle H_{x x} X_{1}, X_{2}\right\rangle \\
\left\langle H_{x x} X_{1}, X_{2}\right\rangle & \left\langle H_{x x} X_{2}, X_{2}\right\rangle
\end{array}\right)+\left\langle H_{x x} X_{3}, X_{3}\right\rangle I
$$

Teorema 4.2 Seja $S \subset \mathbb{R}^{4}$ uma hipersuperficie $C^{k \geqq 2}$ estritamente convexa, difeomorfa a $S^{3}$ tal que $0 \subset B_{S}$. Então o vetor $\alpha(t)=\left(\alpha^{1}(t), \alpha^{2}(t)\right) \subset \mathbb{R}^{2}$ gira em torno da origem sempre no sentido horário ou sempre no sentido anti-horário. Ver figura 4-2.

Prova. Consideremos o vetor $\alpha(t) \wedge \dot{\alpha}(t) \in \mathbb{R}^{3}$ onde a $3^{\circ}$ versor $\vec{k}$ de $\mathbb{R}^{3}$ é ortogonal ao plano que contém a solução $\alpha(t)$. Então temos

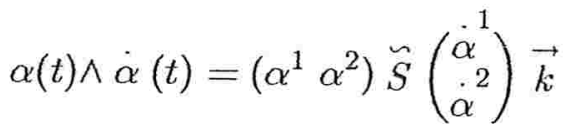

Como $S$ é estritamente convexa, sabemos que $S$ tem curvatura positiva e, portanto,

$$
\begin{aligned}
\operatorname{det} \tilde{S} & =\left|H_{x}\right|^{2}\left\{\operatorname{det}\left(\begin{array}{ll}
\left\langle d N X_{1}, X_{1}\right\rangle & \left\langle d N X_{1}, X_{2}\right\rangle \\
\left\langle d N X_{1}, X_{2}\right\rangle & \left\langle d N X_{2}, X_{2}\right\rangle
\end{array}\right)+\left\langle d N X_{3}, X_{3}\right\rangle \operatorname{tr}(d N)\right\} \\
& =\left|H_{x}\right|^{2}\left\{K_{X_{1} X_{2}}+k_{3} \tilde{H}\right\}>0
\end{aligned}
$$

pois a curvatura seccional $K_{X_{1} X_{2}}$ de $X_{1}$ e $X_{2}$ é positiva e $\tilde{H}$, a curvatura média de $S$ tem o mesmo sinal que a curvatura normal $k_{3}$ na direção $X_{3}$. O traço de $\widetilde{S}$, dado por

$$
\stackrel{\sim}{\operatorname{tr} S}=\left\{\left\langle H_{x x} X_{1}, X_{1}\right\rangle+\left\langle H_{x x} X_{2}, X_{2}\right\rangle+2\left\langle H_{x x} X_{3}, X_{3}\right\rangle\right\}
$$

é positivo se o Hessiano de $H$ é positivo-definido em TS e negativo, caso contrário. Logo, a matriz $\widetilde{S}$ é definida e, portanto, a componente $\vec{k}$ de $\alpha(t) \wedge \dot{\alpha}(t)$ é sempre diferente de zero de modo que $\alpha(t)$ gira em torno da origem sempre no sentido anti-horário se o Hessiano de $H$ é positivo-definido e no sentido horário, caso seja negativo-definido. Ver figura 4-2 


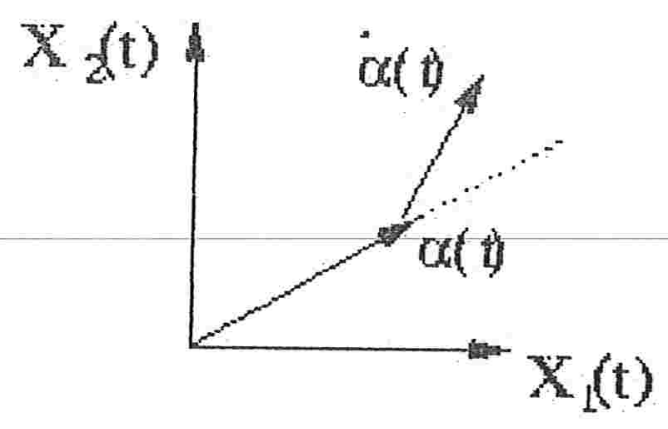

Figura ${ }^{\sim} 4-2$ : No referencial $X_{1} X_{2}$ o vetor $\alpha(t)$ gira sempre no mesmo sentido.

\subsection{Definição do indice de Conley-Zehnder generalizado de ór- bitas periódicas}

O índice de Conley-Zehnder foi introduzido primeiramente em [CZ] e, em poucas palavras, mede o quanto órbitas proximas a uma dada órbita periódica "giram" em torno dela num determinado referencial. Denotaremos por $P$ uma órbita periódica, dada por $x:[0, T] \rightarrow S$ onde $T$ é seu período mínimo e $x([0, T])=P$.

Estaremos também supondo que $S$ é difeomorfo a $S^{3}$ e, portanto, $S$ é simplesmente conexo.

Seja $\xi$ um fibrado linear sobre $S$ tal que para cada $x \in S$, a fibra $\xi_{x} \subset T_{x} S$ é um subespaço de dimensão 2 transversal a $X_{H}(x)$. Além disso, se $\varphi_{t}(x)$ é o fluxo gerado por $X_{H}$, supomos também que $\xi$ é invariante por $D \varphi_{t}$. Seja $v_{D}: D \rightarrow M$ um mergulho do disco compacto $D=\{z \in \mathbb{C}:|z| \leqq 1\}$ em $S$ tal que $v_{D}\left(e^{2 \pi i t}\right)=x(t T)$. Seja $\beta: v_{D}^{*} \xi \rightarrow D \times \mathbb{R}^{2}$ uma trivialização do fibrado $v_{D}^{*} \xi$ sobre $D$. Definimos agora o arco de matrizes simpléticas em dimensão $2, \Phi:[0, T] \rightarrow S p(1)$ pelo fluxo linearizado $D \varphi_{t}$ restrito a $\xi$ ao longo da órbita periódica $x(t)=\varphi_{t}(x(0))$ da seguinte forma

$$
\Phi(t)=\left.\beta\left(e^{2 \pi i t / T}\right) \circ D \varphi_{t}\right|_{\xi_{x(0)}} \circ \beta(1)^{-1}, 0 \leqq t \leqq T
$$

O arco começa na identidade $\Phi(0)=I$ e a órbita periódica é não-degenerada se e só se $\Phi(T)$ não possui o inteiro 1 como autovalor.

Daremos em primeiro lugar uma formulação espectral para o cálculo do índice de ConleyZehnder generalizado que independe da degenerescência da órbita periódica. 
O arco $\Phi$ de matrizes simpléticas satisfaz $\Phi(t+T)=\Phi(t) \Phi(T)$ e, portanto, se definirmos $A(t) \stackrel{\text { def }}{=}-J_{0} \dot{\Phi}(t) \Phi^{-1}(t)$ temos que $A(t)$ é um arco de matrizes simétricas e $A(t)=A(t+T)$.

Definimos agora o operador $L_{A}: H^{1,2}\left(\mathbb{R} / T \mathbb{Z}, \mathbb{R}^{2}\right) \rightarrow L^{2}\left(\mathbb{R} / T \mathbb{Z}, \mathbb{R}^{2}\right)$ dado por $L_{A} \stackrel{\text { def }}{=}$ $-J_{0} \frac{d}{d t}-A(t)$ onde $H^{1,2}\left(\mathbb{R} / T \mathbb{Z}, \mathbb{R}^{2}\right)$ é o espaço de Sobolev dado pelas funções $g: \mathbb{R} / T \mathbb{Z} \rightarrow \mathbb{R}^{2}$ cuja derivada está em $L^{2}\left(\mathbb{R} / T \mathbb{Z}, \mathbb{R}^{2}\right)$. Este operador é auto-adjunto e seu espectro $\sigma\left(L_{A}\right)$ é constituído por um conjunto enumerável de números reais não limitado por cima e nem por baixo. Além disso ker $L_{A}=\{0\}$ se e somente se 1 não é autovalor de $\Phi(T)$.

Seja $v \neq 0$ um autovetor em $L^{2}\left(\mathbb{R} / T \mathbb{Z}, \mathbb{R}^{2}\right)$ associado a $\lambda \in \sigma\left(L_{A}\right)$. Então $v$ satisfaz as seguintes relações

$$
-J_{0} \dot{v}(t)-A(t) v(t)=\lambda v(t), v(0)=v(T)
$$

Isso mostra que $v(t) \neq 0$ para qualquer $t \in[0, T]$ e, como $v$ é periódica, podemos associar a $v$ um winding number $\omega(\lambda, v, A)$. É possivel mostrar as seguintes propriedades

(i) Dois autovetores $v_{1}$ e $v_{2}$ de $L_{A}$, associados ao mesmo autovalor $\lambda$ e linearmente independentes, determinam o mesmo winding number, ou seja, $\omega\left(\lambda, v_{1}, A\right)=\omega\left(\lambda, v_{2}, A\right)$. Desta forma, podemos apenas denotar o winding number por $\omega(\lambda, A)$;

(ii) Para cada número inteiro $k$, existem exatamente dois autovalores $\lambda_{1}$ e $\lambda_{2}$, levando-se em consideraçâo a multiplicidade dos autovalores, tais que $k=\omega\left(\lambda_{1}, A\right)=\omega\left(\lambda_{2}, A\right)$;

(iii) A aplicação $\omega_{A}: \sigma\left(L_{A}\right) \rightarrow \mathbb{Z}$ dada por $\omega_{A}(\lambda)=\omega(\lambda, A)$ é monótono crescente;

Sejam $\alpha(A)$ e $p(A)$ os números inteiros dados por

$$
\begin{aligned}
& \alpha(A)=\max \left\{\omega(\lambda, A) \mid \lambda \in \sigma\left(L_{A}\right) \cap(-\infty, 0)\right\} \\
& p(a)=\left\{\begin{array}{l}
0 \text { se existe } \lambda \in \sigma\left(L_{A}\right) \cap[0, \infty) \text { tal que } \omega(\lambda, A)=\alpha(A) \\
1 \text { caso contrário }
\end{array}\right.
\end{aligned}
$$

Tais propriedades aparecem com mais detalhes em [HZW2] e [HZW3].

Definimos $\tilde{\mu}(A)=2 \alpha(A)+p(A)$. É possível mostrar que esta definição independe da trivialização $\beta$. Como estamos supondo que $S$ é difeomorfo a $S^{3}$, o cálculo de $\tilde{\mu}(A)$ também independe do mergulho $v_{D}$. O índice de Conley-Zehnder generalizado da órbita periódica $P$ é definido por $\tilde{\mu}_{C Z}(P)=\tilde{\mu}(A)$.

Uma definição mais geométrica do índice de Conley-Zehnder para órbitas periódicas não- 
degeneradas é apresentada a seguir: considere o arco de matrizes simpléticas $\Phi:[0, T] \rightarrow S p(\mathcal{I})$ definido acima. Seja $z \in \mathbb{C} \backslash\{0\}$ e $\rho(t)$ um argumento contínuo para a solução $z(t)=\Phi(t) z$, ou seja, $\rho(t)$ é uma função real contínua em $[0, T]$ tal que $e^{2 \pi i \rho(t)}=\frac{z(t)}{|z(t)|}$. Seja $\Delta(z) \stackrel{\text { def }}{=} \rho(T)-\rho(0)$ e definimos o intervalo associado ao arco $\Phi$ por $I(\Phi)=\{\Delta(z): z \in \mathbb{C} \backslash\{0\}\}$. O conjunto $I(\Phi)$ é um intervalo com comprimento menor que $1 / 2 \mathrm{e}$, portanto, está contido entre 2 inteiros ou contém um inteiro. Podemos definir, então

$$
\tilde{\mu}(\Phi)= \begin{cases}2 k+1 & \text { se } I(\Phi) \subset(k, k+1) \\ 2 k & \text { se } k \in I(\Phi)\end{cases}
$$

Como $M$ é difeomorfa a $S^{3}, \widetilde{\mu}(\Phi)$ não depende do mergulho $v_{D}$. Além disso também não depende da trivialização $\beta$ (ver [HZW2]). Assim, podemos definir um novo índice $\widetilde{\mu}_{2}(P$ ) da órbita periódica não-degenerada $P$, por $\tilde{\mu}_{2}(P)=\tilde{\mu}(\Phi)$.

Proposição 4.1 Para órbitas periódicas não-degeneradas, temos que $\widetilde{\mu}_{C Z}(P)=\widetilde{\mu}_{2}(P)$.

Prova. Ver $[\mathrm{HK}]$.

O cálculo de $\widetilde{\mu}_{2}(P)$ pode também ser feito para órbitas periódicas degeneradas, porém nem sempre dá exatamente o índice de Conley-Zehnder $\tilde{\mu}_{C Z}(P)$. Apesar disso, temos uma boa estimativa, conforme a seguinte proposição

Proposição 4.2 Para órbitas periódicas degeneradas, temos $\widetilde{\mu}_{C Z}(P)=\tilde{\mu}_{2}(P)$ ou $\widetilde{\mu}_{C Z}(P)=\widetilde{\mu}_{2}$ $(P)-1$.

Prova. Sabemos que quando uma órbita periódica é degenerada, o operador $L_{A}$ possui algum autovetor $v \neq 0$ em $L^{2}\left(\mathbb{R} / T \mathbb{Z}, \mathbb{R}^{2}\right)$ associado ao autovalor 0 . Por (4.8) sabemos que $v$ satisfaz $\dot{v}(t)=\dot{\Phi}(t) \Phi^{-1}(t) v(t)$ e, portanto, temos que $v(t)=\Phi(t) v(0)$. Como $v$ é periódica, temos que $\tilde{\mu}_{2}(P)=2 \omega(0, A)$. Das propriedades de $\omega(\lambda, A)$ e da definição de $\alpha(A)$ concluímos que $\alpha(A)=\omega(0, A)$ (neste caso $p(A)=0$ ) ou $\alpha(A)=\omega(0, A)-1$ (neste caso $p(A)=1$ ). Então temos $\tilde{\mu}_{C Z}(P)=\tilde{\mu}(A)=2 \omega(0, A)=\widetilde{\mu}_{2}(P)$ ou $\widetilde{\mu}_{C Z}(P)=\tilde{\mu}(A)=2 \omega(0, A)-1=\widetilde{\mu}_{2}(P)-1$.

Uma definição mais axiomática e geral do índice de Conley-Zehnder pode ser vista em [CZ]. 


\subsection{Equivalência do Fluxo Hamiltoniano em Hipersuperficies Regulares}

Nesta seção, mostraremos que o fluxo Hamiltoniano em um determinado nível de energia $S$ depende fundamentalmente de $S$ e não da função Hamiltoniana que o determina.

Proposição 4.3 Seja $S$ uma hipersuperfície $C^{k \geqq 2}$, conexa e orientável em $\mathbb{R}^{4}$. Sejam $H, G$ : $\mathbb{R}^{4} \rightarrow \mathbb{R}$, duas funções $C^{k \geqq 2}$ tais que $S \subset H^{-1}\left(c_{1}\right)$ e $S \subset G^{-1}\left(c_{2}\right)$ e para todo $x \in S$, x é ponto regular de $H$ e $G$. Então o fluxo Hamiltoniano associado às funções $H$ e $G$ são iguais em $S$, a menos de uma reparametrização no tempo.

Prova. Sabemos que o fluxo Hamiltoniano preserva níveis de energia, portanto, vamos mostrar que para todo $x \in S$, temos $X_{H}(x)=f(x) X_{G}(x)$ para alguma função diferenciável não nula $f: S \rightarrow \mathbb{R}$.

Seja $N: S \rightarrow S^{3}$ uma orientação em $S$, ou seja, $N$ é uma aplicação contínua que associa a cada $x \in S$ um vetor unitário $N(x)$ normal a $S$ em $x$.

Para todo $x \in S$ sabemos, por hipótese, que os vetores gradiente $H_{x}(x)$ e $G_{x}(x)$ são diferentes de zero. Além disso eles devem ser paralelos pois ambos são paralelos a $N(x)$. Portanto $G_{x}(x)=f(x) H_{x}(x)$ onde $f$ é uma função deferenciável e diferente de zero.

Dada uma solução $x: I \subset \mathbb{R} \rightarrow S$ do fluxo Hamiltoniano

$$
\frac{d x}{d t}=J_{0} H_{x}(x), x(0)=x_{0}
$$

então existe um difeomorfismo $k: J \subset \mathbb{R} \rightarrow I, k(0)=0$, tal que $\tilde{x}(t) \stackrel{\text { def }}{=} x(k(t))$ é solução de

$$
\frac{d \tilde{x}}{d t}=J_{0} G_{x}(\tilde{x}), \tilde{x}(0)=x_{0}
$$

Para isso, basta definir $k$ como solução de

$$
\dot{k}=f(x(k(t)))
$$

e observar que $\frac{d \tilde{x}}{d t}=\frac{d x(k(t))}{d t} \dot{k}=f(x(k(t))) J_{0} H_{x}(x(k(t)))=J_{0} G_{x}(\tilde{x})$. As soluções de $X_{H}(x)$ e $X_{G}(x)$ são, portanto, iguais após a reparametrização $k$. 
O fluxo linearizado de $X_{H}$ restrito a $T S$, ao longo de uma solução $x(t)$, é dado por

$$
\dot{y}=J_{0} H_{x x}(x(t)) y
$$

onde $y(t) \in T_{x(t)} S$ para todo $t \in I$. Analogamente, o fluxo linearizado de $X_{G}$ restrito a $T S$ ao longo de uma solução $\tilde{x}(t)$ é dado por

$$
\dot{y}=J_{0} G_{x x}(\breve{x}(t)) y
$$

onde $\tilde{y}(t) \in T_{\widetilde{x}(t)} S$.

Vamos mostrar agora que o fluxo linearizado independe da função Hamiltoniana que determina a superfície $S$ no plano ortogonal à direção do campo Hamiltoniano.

Para isso, lembremos a trivialização de $T S$ já apresentada anteriormente. Em cada ponto $x \in S, T=\left\{X_{1}(x), X_{2}(x), X_{3}(x)\right\}$ é uma base ortonormal de $T_{x} S$ tal que $X_{3}(x)$ está na mesma direção do campo Hamiltoniano associado a $S$. Sejam $\alpha_{i}, i=1,2,3$ as componentes de $y(t)$ na base $T$.

Proposição 4.4 Considerando as mesmas hipóteses e notações da proposição 4.3 temos que se $y(t)=\left(\alpha_{1}(t), \alpha_{2}(t), \alpha_{3}(t)\right) \in T_{x(t)} S$ é solução de (4.12) e $\widetilde{y}(t)=\left(\tilde{\alpha}_{1}(t), \widetilde{\alpha}_{2}(t), \tilde{\alpha}_{3}(t)\right) \in T_{\tilde{x}_{(t)}} S$ é solução de (4.13), $\tilde{y}(0)=y(0)$, então $\tilde{\alpha}_{1}(t)=\alpha_{1}(k(t))$ e $\tilde{\alpha}_{2}(t)=\alpha_{2}(k(t))$, onde $k$ foi definida em (4.11).

Prova. Seja

$$
R_{x} \stackrel{d e f}{=} J\left[\left(\begin{array}{ll}
\left\langle d N X_{1}, X_{1}\right\rangle & \left\langle d N X_{1}, X_{2}\right\rangle \\
\left\langle d N X_{1}, X_{2}\right\rangle & \left\langle d N X_{2}, X_{2}\right\rangle
\end{array}\right)+\left\langle d N X_{3}, X_{3}\right\rangle I\right]_{x}
$$

Lembramos que

$$
\left(\begin{array}{c}
\dot{\alpha}_{1} \\
\dot{\alpha}_{2}
\end{array}\right)=-\left|H_{x}\right| R_{x(t)}\left(\begin{array}{c}
\alpha_{1} \\
\alpha_{2}
\end{array}\right)
$$


Definindo-se $\beta_{1}(t)=\alpha_{1}(k(t))$ e $\beta_{2}(t)=\alpha_{2}(k(t))$, temos

$$
\begin{aligned}
\left(\begin{array}{c}
\dot{\beta}_{1} \\
\dot{\beta}_{2}
\end{array}\right) & =\dot{k}\left(\begin{array}{c}
\dot{\alpha}_{1}(k(t)) \\
\dot{\alpha}_{2}(k(t))
\end{array}\right)=-\dot{k}\left|H_{x}\right| R_{x(k(t))}\left(\begin{array}{c}
\alpha_{1}(k(t)) \\
\alpha_{2}(k(t))
\end{array}\right) \\
& =-\left|G_{x}\right| R_{\widetilde{x}(t)}\left(\begin{array}{c}
\beta_{1} \\
\beta_{2}
\end{array}\right)
\end{aligned}
$$

e, portanto, $\beta_{i}(t)=\tilde{\alpha}_{i}(t)$ para $i=1,2$.

As proposições 4.3 e 4.4 dizem que além de as soluções de $X_{H}(x)$ e $X_{G}(x)$ serem iguais em $S$, após uma reparametrização no tempo, a projeção no plano $\left\{X_{1}, X_{2}\right\}$ do fluxo linearizado em $T S$ ao longo de uma solução é também igual após feita a mesma reparametrização no tempo. Usaremos a expressão "fluxo Hamiltoniano em $S$ " quando não for necessário explicitar uma particular função Hamiltoniana $H$ tal que $S$ e $H$ satisfiçam as hipóteses do teorema 4.3.

Vimos que o cálculo do índice de Conley-Zehnder de uma órbita periódica depende da projeção do fluxo linearizado no plano gerado por $X_{1}$ e $X_{2}$. A Proposição 4.4 garante que este cálculo não sofre nenhuma alteração com a mudança da função Hamiltoniana escolhida.

Se $S$ é uma hipersuperífice estritamente convexa e difeomorfa a $S^{3}$ tal que a origem esteja contida em seu interior, podemos considerar o fibrado $\xi$ sobre $S$, também chamado de estrutura de contato, definido por

$$
\xi=\left\{(x, v) \in T S, \lambda_{0}(x) v=0\right\}
$$

A fibra $\xi_{p}$, em cada ponto $p \in S$, é bi-dimensional e tranversal ao campo Hamiltoniano $X_{H}$. Ver definição de $\lambda_{0}$ em (8.1). Logo $\xi$ pode ser trivializada conforme (4.3).

Na direção de $X_{H}$ o fluxo linearizado do campo Hamiltoniano muda conforme a escolha da função Hamiltoniana que tem $S$ como conjunto regular, e por isso, em geral, a estrutura de contato $\xi$ não é invariante pelo fluxo. Entretanto, podemos sempre escolher $H: \mathbb{R}^{4} \rightarrow \mathbb{R}$ tal que $S=H^{-1}(1)$ e o fluxo Hamiltoniano associado a $H$ preserva a estrutura de contato $\xi$.

Para o cálculo do índice de Conley-Zehnder de uma órbita periódica $P$ contida em $S$, devemos trivializar a estrutura de contato $\xi$ sobre um disco compacto mergulhado $D$ tal que $P=\partial D$. Vimos que a base $\tilde{T}=\left\{\tilde{X}_{1}, \tilde{X}_{2}\right\}$, definida em (4.3), trivializa o fibrado $\xi$ em qualquer subconjunto de $S$ e, em particular, em $D$. Essa trivialização pode ser simplética fazendo-se uma 
mudança de escala (considerando a forma simplética canônica em $\mathbb{R}^{2}$ e a forma simplética $d \lambda_{0}$ restrita a $\xi$ ). Como usamos apenas o argumento do vetor na base $\widetilde{T}$ para o cálculo do índice de Conley-Zehnder, essa mudança de escala não é relevante. As coordenadas na base $\tilde{T}$ são iguais às coordenadas na base $\left\{X_{1}, X_{2}\right\}$. Portanto, podemos calcular o índice de Conley-Zehnder apenas olhando para a projeção do fluxo linearizado sobre o plano gerado por $X_{1}$ e $X_{2}$.

Por exemplo, consideremos o elipsóide $E=H^{-1}(1)$ onde $H=x_{1}^{2}+p_{1}^{2}+\frac{x_{2}^{2}+p_{2}^{2}}{r^{2}}$ e $r^{2}$ é um irracional maior que 1. O fluxo Hamiltoniano $X_{H}$ em $E$ possui exatamente 2 órbitas periódica dadas por $P_{1}=\left\{x_{1}^{2}+p_{1}^{2}=1, x_{2}=p_{2}=0\right\}$ e $P_{2}=\left\{x_{2}^{2}+p_{2}^{2}=r^{2}, x_{1}=p_{1}=0\right\}$. Ambas as órbitas são não-degeneradas e, portanto, podemos calcular $\mu_{C Z}\left(P_{1}\right)$ e $\mu_{C Z}\left(P_{2}\right)$ pelo método geométrico descrito acima. A projeção do fluxo linearizado sobre o plano gerado por $X_{1}$ e $X_{2}$ ao longo das órbitas periódicas $P_{1}$ e $P_{2}$ é dada pela equação (4.6)

$$
\left(\begin{array}{l}
\dot{\alpha}_{1} \\
\dot{\alpha}_{2}
\end{array}\right)=\left(\begin{array}{ll}
0 & -\left(2+\frac{2}{r^{2}}\right) \\
2+\frac{2}{r^{2}} & 0
\end{array}\right)\left(\begin{array}{l}
\alpha_{1} \\
\alpha_{2}^{\prime}
\end{array}\right)
$$

O período de $P_{1}$ é $\pi$. A variação de argumento de uma solução no período $\pi$ é $\left(1+\frac{1}{r^{2}}\right) 2 \pi$. Como $2<2\left(1+\frac{1}{r^{2}}\right)<4$, temos que $\mu_{C Z}\left(P_{1}\right)=3$. O período de $P_{2}$ é $\pi r^{2}$ e a variação de argumento de uma solução nesse período é $\left(1+r^{2}\right) 2 \pi$. Logo $\mu_{C Z}\left(P_{2}\right)=2 k+1$, onde $k$ é o inteiro que satisfaz $k<1+r^{2}<k+1$.

Fazendo-se $r=1$ no exemplo anterior obtemos a esfera $S^{3}$. Todas as órbitas em $S^{3}$ são periódicas e degeneradas. Pela simetria esférica, o índice de Conely-Zehnder $\mu_{C Z}(P)$ independe da escolha da órbita periódica $P$ e, por ser degenerada, não podemos calculá-lo utilizando o método geométrico. Mas podemos fazer uma estimativa conforme a Proposição 4.2. Temos nesse caso $\tilde{\mu}_{2}(P)=4$ e, portanto $\mu_{C Z}(P)=3$ ou $\mu_{C Z}(P)=4$. Veremos mais adiante que hipersuperfícies estritamente convexas, como o elipsóide e a esfera, possuem sempre uma órbita periódica com índice de Conley-Zehnder igual a 3. Portanto, $\mu_{C Z}(P)=3$ para todas as órbitas periódicas de $S^{3}$. O método espectral também é um forma simples e direta de se calcular o índice de Conley-Zehnder das órbitas periódicas em $S^{3}$. 


\subsection{Conjuntos invariantes em hipersuperficies de $\mathbb{R}^{4}$}

Nesta seção apresentaremos uma condição para a não existência de órbitas periódicas em hipersuperfícies de $\mathbb{R}^{4}$.

Teorema 4.3 Seja $S \subset \mathbb{R}^{4}$ uma hipersuperfície $C^{k \geqq 1}$, orientável e conexa. Seja $K \subset S$ um compacto. Suponhamos que exista um vetor $N \in \mathbb{R}^{4}, N \neq 0$, tal que para todo $x \in K$, temos $\langle N(x), N\rangle \neq 0$, onde $N: S \rightarrow S^{3}$ é um campo de vetores unitário contínuo normal a $S$. Então o fluxo Hamiltoniano em $S$ nẫo possui órbitas totalmente contidas em K. Em particular, não existem órbitas periódicas em $K$.

Prova. Seja $H: \mathbb{R}^{4} \rightarrow \mathbb{R}$ uma função Hamiltoniana $C^{k \geqq 1}$, tal que $S \subset H^{-1}(c)$ para algum $c \in \operatorname{Im}(H)$ tal que todo ponto $x \in S, x$ é ponto regular de $H$.

Seja $X_{i}=A_{i} N, i=1,2,3$, onde $A_{i}$ são matrizes definidas em (4.1). Então o conjunto $X \stackrel{\text { def }}{=}\left\{X_{1}, X_{2}, X_{3}, N\right\}$ forma uma base ortonormal para $\mathbb{R}^{4}$. Se $x \in S$, então $x=x_{1} X_{1}+x_{2} X_{2}+$ $x_{3} X_{3}+x_{4} N$. Seja $x(t)=\left(x_{1}(t), x_{2}(t), x_{3}(t), x_{4}(t)\right) \in S$ uma solução do fluxo Hamiltoniano associado à função $H$, cujas coordenadas estão na base $X$. Temos

$$
\begin{gathered}
\dot{x}(t)=\dot{x}_{1}(t) X_{1}+\dot{x}_{2}(t) X_{2}+\dot{x}_{3}(t) X_{3}+\dot{x}_{4}(t) N \\
\dot{x}_{3}(t)=\left\langle\dot{x}(t), X_{3}\right\rangle
\end{gathered}
$$

Por hipótese $\langle N(x), N\rangle \neq 0$ para todo $x \in U_{K}$, uma vizinhança aberta de $K$ em $S$. Como $A_{3}^{t}=-A_{3}$ e $A_{3} A_{3}=-I_{4 \times 4}$, temos

$$
\begin{aligned}
\dot{x}_{3}(t) & =\left\langle\dot{x}(t), X_{3}\right\rangle=\left\langle A_{3} H_{x}(x(t)), X_{3}\right\rangle=\left\langle H_{x}(x(t)), A_{3}^{t} A_{3} N\right\rangle \\
& =\left\|H_{x}(x(t))\right\|\left\langle\frac{H_{x}(x(\tilde{t}))}{\left\|H_{x}(x(\tilde{u}))\right\|}, N\right\rangle= \pm\left\|H_{x}(x(t))\right\|\langle N(x), N\rangle \neq 0
\end{aligned}
$$

e, portanto, $\dot{x}_{3}(t) \neq 0$. Logo $x_{3}(t)$ é uma função monótona em $U_{K}$. Suponhamos, sem perda de generalidade, que $\dot{x}_{3}(t)>0$ para toda solução em $U_{K}$. Ver figura 4-3.

Suponhamos que $x(t) \in K$ para todo $t>0$. Como $K$ é compacto, existem números reais $t_{k} \stackrel{k \rightarrow \infty}{\longrightarrow} \infty$ tal que $x\left(t_{k}\right) \stackrel{k \rightarrow \infty}{\longrightarrow} z \in K$. Seja $x_{3}(x)$ a coordenada $x_{3}$ do ponto $x$ na base $X$. Então, 


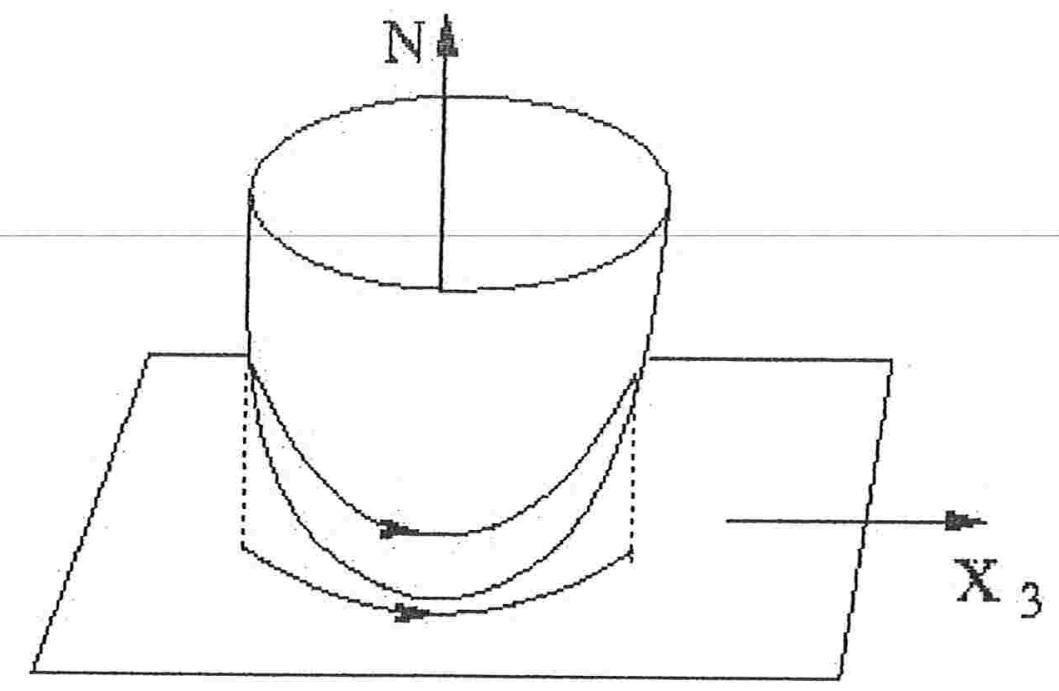

Figura 4-3: Na direção $X_{3}$ o fluxo é monótono em $K$.

por continuidade, $x_{3}\left(t_{k}\right) \stackrel{k \rightarrow \infty}{\longrightarrow} x_{3}(z)$. Mas $X_{H}(z) \neq 0$ pois $z$ é ponto regular de $H$, logo existe uma vizinhança $U_{z} \subset U_{K}$ de $z$ em $S$ e $\delta>0$ tais que $\varphi\left(\delta, U_{z}\right) \cap U_{z}=\emptyset$. Podemos também supor que $\varphi\left(\delta, U_{z}\right) \subset U_{K}$ e que $x_{3}(x)>x_{3}(y)$ para todo $x \in \varphi\left(\delta, U_{z}\right)$ e $y \in U_{z}$ pois $\dot{x}_{3}>0$ em todas as soluções contidas em $U_{K}$. Portanto existe uma seqüência de números reias $s_{k} \stackrel{k \rightarrow \infty}{\longrightarrow} \infty$ tal que $x\left(s_{k}\right) \in \varphi\left(\delta, U_{z}\right)$ onde $x_{3}\left(s_{k}\right)>x_{3}(z)$, uma contradição.

Corolário 4.1 Seja $S$ uma hipersuperfície representada como gráfico de uma função $f: U \subset$ $\mathbb{R}^{3} \rightarrow \mathbb{R}, C^{k \geqq 1}$, ou seja, $S=\left\{\left(x_{1}, x_{2}, x_{3}, x_{4}\right) \in \mathbb{R}^{4} \mid\left(x_{1}, x_{2}, x_{3}\right) \in U, x_{4}=f\left(x_{1}, x_{2}, x_{3}\right)\right\}$ onde $U$ é um aberto conexo. Então o fluxo Hamiltoniano não apresenta órbitas periódicas em qualquer compacto contido em $S$.

Prova. Seja $N=(0,0,0,1)$ nas coordenadas $\left(x_{1}, x_{2}, x_{3}, x_{4}\right)$. O vetor normal a $S$ em $x$ pode ser expressado por $N(x)=\frac{1}{\left\|\left(-f_{x_{1}}(x),-f_{x_{2}}(x),-f_{x_{3}}(x), 1\right)\right\|}\left(-f_{x_{1}}(x),-f_{x_{2}}(x),-f_{x_{3}}(x), 1\right) \neq 0$ pois a função $f$ é $C^{1}$. Logo $\langle N(x), N\rangle \neq 0$ para todo $x \in S$. Então $S$ satisfaz as hipóteses do teorema 4.3 e, portanto, não existem órbitas periódicas do fluxo Hamiltoniano em $S$.

Proposição 4.5 Seja $S$ uma hipersuperfície $C^{k \geqq 1}$, orientável, em $\mathbb{R}^{4}$ e $H: \mathbb{R}^{4} \rightarrow \mathbb{R}$ uma função tal que $S=H^{-1}(c)$ para algum valor regular c. Seja $\varphi(t, x)$ o fluxo Hamiltoniano associado a $H$. Seja $K \subset S$ um compacto com as seguintes propriedades: (i) $K$ é difeomorfo 


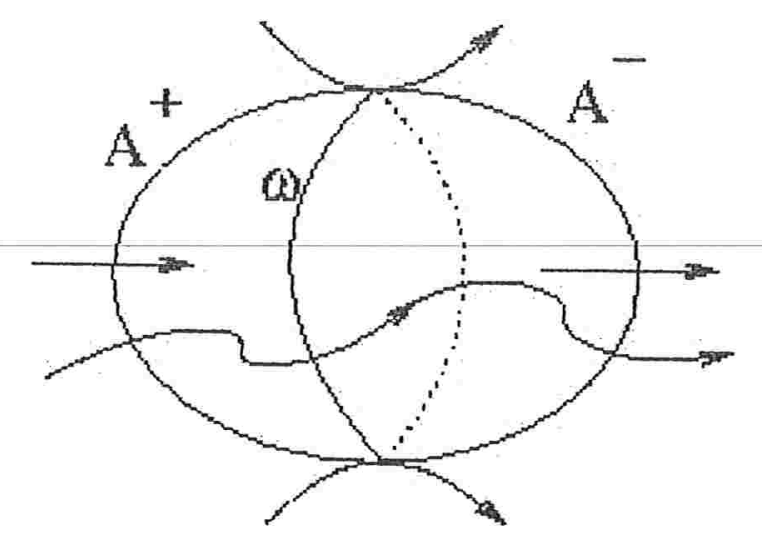

Figura 4-4: Toda órbita que entra no compacto $K$ por $A^{+}$deve sair de $K$ por $A^{-}$. a $B^{3}$, a bola unitária de dimensão 3 ; (ii) $\partial K=A^{+} \cup A^{-} \cup \omega$, onde $A^{+}$e $A^{-}$são difeomorfos a $D^{2}$ e $\omega$ é difeomorfo a $S^{1}$. O campo $X_{H}(x)$ é transversal a $\partial K$ para todo $x \in A^{+} \cup A^{-}$. Se $x \in A^{+}$então $\varphi(t, x) \in K$ para todo $t>0$ suficientemente pequeno e $\varphi(t, x) \notin K$ para todo $t<0$ suficientemente pequeno. Analogamente se $x \in A^{-}$então $\varphi(t, x) \notin K$ para todo $t>0$ suficientemente pequeno e $\varphi(t, x) \in K$ para todo $t<0$ suficientemente pequeno. O conjunto $\omega \dot{e}$ formado pelos pontos $x$ cuja órbita tangencia $K$ e para todo tempo $t \neq 0$ suficientemente pequeno $\varphi(t, x) \notin K$. (iii) Existe um vetor $N$ tal que $\langle N(x), N\rangle \neq 0$ para todo $x \in K$, onde $N(x)$ é um campo de vetores contínuo normal a $S$; Entẫo existe um difeomorfismo $\varphi_{K}: A^{+} \rightarrow A^{-}$que descreve o fluxo $\varphi(t, x)$ em $\stackrel{\circ}{K}$, ou seja, se $x \in A^{+}$, existe $t_{x}>0$ tal que $\varphi_{K}(x)=\varphi\left(t_{x}, x\right) \in A^{-}$ e $\varphi(t, x) \in \stackrel{\circ}{K}$ para todo $0<t<t_{x}$. Ver figura $4-4$.

Prova. Sabemos, pelo Teorema 4.3, que não existem órbitas totalmente contidas em $K$ e, portanto, a solução $x(t)$ que passa por $x \in A^{+}$deve sair de $K$ e, pela hipótese feita sobre $\omega$, $x(t)$ sai de $K$ por $A^{-}$, ou seja existe $t_{x}>0$ tal que $x\left(t_{x}\right) \in A^{-}$e $x\left(t_{x}\right) \in \stackrel{\circ}{K}$ para $0<t<t_{x}$. Seja $\varphi_{K}: A^{+} \rightarrow A^{-}$onde $\varphi_{K}(x) \stackrel{\text { def }}{=} x\left(t_{x}\right)=\varphi\left(t_{x}, x\right)$. Obviamente $\varphi_{K}$ está bem definida $e$, considerando-se o campo $-X_{H}$, vemos que $\varphi_{K}$ é bijetora. A transversalidade do campo em $A^{+} \cup A^{-}$e a regularidade de $\partial K$ nos garante que $\varphi_{K}$ é um difeomorfismo local, e, portanto, $\varphi_{K}$ é um difeomorfismo. 


\section{Capítulo 5}

\section{Estimativa do índice de}

\section{Conley-Zehnder para órbitas}

\section{periódicas que passam nas}

\section{proximidades de um sela-centro}

Seja $p_{c}$, diferente da origem, um ponto de equilíbrio do tipo sela-centro para o fluxo Hamiltoniano gerado por $H: \mathbb{R}^{4} \rightarrow \mathbb{R}$ onde $H$ é uma função real-analítica. Como já vimos, podemos aplicar o Teorema de Moser e encontrar coordenadas numa vizinhança de $p_{c}$ onde o fluxo se apresenta numa forma bastante simplificada. Utilizaremos essas coordenadas para estimarmos o índice de Conley-Zehnder de órbitas periódicas que passam perto do equilíbrio $p_{c}$.

Suponhamos $H\left(p_{c}\right)=0$. Sabemos que $H_{x}\left(p_{c}\right)=0$ e, portanto, podemos escrever a função $H$ da seguinte forma

$$
H(x)=\frac{1}{2}\left\langle A\left(x-p_{c}\right),\left(x-p_{c}\right)\right\rangle+R_{0}\left(x-p_{c}\right)
$$

onde $x=\left(x_{1}, x_{2}, x_{3}, x_{4}\right)$ e $\left\|R_{0}(x)\right\| \leqq r_{0}\|x\|^{3}$. A matriz $A$ é tal que $J A$ possui um par de autovalores reais $\pm \bar{\alpha}(\bar{\alpha}>0)$, e um par de autovalores imaginários puros $\pm \bar{\omega} i(\bar{\omega}>0)$.

Seja $U$ uma vizinhança de $p_{c}$ em $\mathbb{R}^{4}$ onde vale o Teorema de Moser. Seja $\varphi: V \rightarrow U$ 
a mudança de coordenadas que conjunga o fluxo gerado por $H$ em $U$ ao fluxo gerado pelo Hamiltoniano $K: V \rightarrow \mathbb{R}$ dado por

$$
K\left(I_{1}, I_{2}\right)=-\bar{\alpha} I_{1}+\bar{\omega} I_{2}+\mathcal{O}\left(I_{1}^{2}+I_{2}^{2}\right)
$$

onde $I_{1}=q_{1} p_{1}$ e $I_{2}=\frac{q_{2}^{2}+p_{2}^{2}}{2}$. As coordenadas em $V$ são $y=\left(q_{1}, q_{2}, p_{1}, p_{2}\right)$.

Suponhamos que exista um subconjunto $S \subset \mathbb{R}^{4}$ que satisfaça as seguintes propriedades:

(i) $S \subset H^{-1}(0)$;

(ii) $p_{c} \in S$;

(iii) $S$ é homeomorfo a $S^{3}$;

(iv) $S$ é invariante pelo fluxo Hamiltoniano gerado por $H$;

(v) $p_{c}$ é o único ponto crítico de $S$, ou seja, $H_{x}(q) \neq 0$ para todo $q \in S, q \neq p_{c}$;

(vi) $S \backslash\left\{p_{c}\right\}$ é estritamente convexa.

O conjunto $S_{0} \stackrel{\text { def }}{=} S \backslash\left\{p_{c}\right\}$ é, portanto, uma hipersuperfície regular de $\mathbb{R}^{4}$, invariante pelo fluxo de $H$. Podemos calcular os vetores $X_{i}, i=1,2,3$, que trivializam $T S_{0}$ conforme (4.2). Queremos estimar $X_{i}$ sobre a variedade estável de $p_{c}$.

Sabemos que em $V$, a variedade estável local do sela-centro é dada pelo conjunto $\tilde{W}_{V}^{s}=\left\{q_{1} \in\right.$ $\left.\mathbb{R} \mid\left(q_{1}, 0,0,0\right) \in V\right\}$, ou seja, é um segmento de reta $r$ em $V$ gerado pelo vetor $v_{1}=(1,0,0,0)$. Vamos supor que o ramo da variedade estável de $p_{c}$ contido em $S_{0}$ seja dado pelos pontos em $\tilde{W}_{V}^{s}$ que satisfazem $q_{1}>0$. O caso $q_{1}<0$ é análogo. Logo, pela aplicação $\varphi$, que é um difeomorfismo, a variedade estável local de $p_{c}$ em $S_{0}$ é um conjunto que se aproxima perto da origem de uma semi-reta $s$ gerada pelo vetor $u_{1} \stackrel{\text { def }}{=} M v_{1}$ onde $M \stackrel{\text { def }}{=} D \varphi(0)$. Sabemos que $\varphi(y)=p_{c}+M y+L_{0}(y)$, onde $\left\|L_{0}(y)\right\| \leqq l_{0}\|y\|^{2}, l_{0}>0$, e, em $S_{0}$, a variedade estável de $p_{c}$ é dada localmente por $W_{S_{0}}^{s} \stackrel{\text { def }}{=} \varphi\left(W_{V}^{s}\right)=\left\{p_{c}+q_{1} u_{1}+Z_{0}\left(q_{1}\right), 0 \leqq q_{1} \leqq \delta\right\}$ onde $\left\|Z_{0}(x)\right\| \leqq z_{0} x^{2}$, $z_{0}>0$. Seja $N(x)$ o vetor normal a $S_{0}$ no ponto $x \in S_{0}$.

Lema 5.1 Para $x \in W_{S_{0}}^{s}$, temos $X_{i}(x) \stackrel{x \rightarrow p_{c}}{\longrightarrow} X_{i}^{\infty} \stackrel{\text { def }}{=} \frac{A_{i} A u_{1}}{\left\|A u_{1}\right\|}$ e $N(x) \stackrel{x \rightarrow p_{c}}{\longrightarrow} \frac{A u_{1}}{\left\|A u_{1}\right\|}$.

Prova. Por (5.1) temos que $H_{x}(x)=A\left(x-p_{c}\right)+R_{1}\left(x-p_{c}\right)$ onde $\left\|R_{1}(x)\right\| \leqq r_{1}\|x\|^{2}$. Então, se $x \in W_{S_{0}}^{s}$, temos

$$
H_{x}\left(x\left(q_{1}\right)\right)=q_{1} A u_{1}+A Z_{0}\left(q_{1}\right)+R_{1}\left(q_{1} u_{1}+Z_{0}\left(q_{1}\right)\right)
$$


Mas

$$
\begin{aligned}
\left\|A Z_{0}\left(q_{1}\right)\right\| & \leqq\|A\|\left\|Z_{0}\left(q_{1}\right)\right\| \leqq \tilde{z}_{0} q_{1}^{2} \\
\left\|R_{1}\left(q_{1} u_{1}+Z_{0}\left(q_{1}\right)\right)\right\| & \leqq r_{1}\left\|q_{1} u_{1}+Z_{0}\left(q_{1}\right)\right\|^{2} \\
\left\|q_{1} u_{1}+Z_{0}\left(q_{1}\right)\right\| & \leqq \tilde{r}_{0} q_{1}+z_{0} q_{1}^{2} \leqq \hat{r}_{0} q_{1}
\end{aligned}
$$

Usando (5.3) e (5.4) temos que $H_{x}\left(x\left(q_{1}\right)\right)=q_{1} A u_{1}+R_{2}\left(q_{1}\right)$ onde $\left\|R_{2}\left(q_{1}\right)\right\| \leqq r_{2} q_{1}^{2}$ e $r_{2}=\hat{r}_{0}^{2}$ $+\tilde{z_{0}}>0$. Logo

$$
\begin{aligned}
& X_{i}\left(x\left(q_{1}\right)\right)=\frac{A_{i}\left(q_{1} A u_{1}+R_{2}\left(q_{1}\right)\right)}{\left\|q_{1} A u_{1}+R_{2}\left(q_{1}\right)\right\|}=\frac{q_{1} A_{i} A u_{1}}{\left\|q_{1} A u_{1}+R_{2}\left(q_{1}\right)\right\|}+\frac{A_{i} R_{2}\left(q_{1}\right)}{\left\|q_{1} A u_{1}+R_{2}\left(q_{1}\right)\right\|} \\
& N\left(x\left(q_{1}\right)\right)=\frac{H_{x}\left(x\left(q_{1}\right)\right)}{\left\|H_{x}\left(x\left(q_{1}\right)\right)\right\|}=\frac{q_{1} A u_{1}}{\left\|q_{1} A u_{1}+R_{2}\left(q_{1}\right)\right\|}+\frac{R_{2}\left(q_{1}\right)}{\left\|q_{1} A u_{1}+R_{2}\left(q_{1}\right)\right\|}
\end{aligned}
$$

Denotando $c_{1}=\frac{q_{1} A u_{1}}{\left\|q_{1} A u_{1}+R_{2}\left(q_{1}\right)\right\|}$ e $c_{2}=\frac{R_{2}\left(q_{1}\right)}{\left\|q_{1} A u_{1}+R_{2}\left(q_{1}\right)\right\|}$ vamos mostrar que $c_{1} \rightarrow \frac{A u_{1}}{\left\|A u_{1}\right\|}$ e $c_{2} \rightarrow 0$ quando $q_{1} \rightarrow 0$. Temos

$$
\begin{aligned}
\left\|c_{1}-\frac{A u_{1}}{\left\|A u_{1}\right\|}\right\| & =\left\|\frac{q_{1} A u_{1}}{\left\|q_{1} A u_{1}+R_{2}\left(q_{1}\right)\right\|}-\frac{q_{1} A u_{1}}{\left\|q_{1} A u_{1}\right\|}\right\| \\
& =\frac{\left\|q_{1} A u_{1}+R_{2}\left(q_{1}\right)\right\|-\left\|q_{1} A u_{1}\right\| \|}{\left\|q_{1} A u_{1}+R_{2}\left(q_{1}\right)\right\|}
\end{aligned}
$$

Usando a relação $|\|m\|-\|n\|| \leqq\|m-n\|$ temos $\left|\left\|q_{1} A u_{1}+R_{2}\left(q_{1}\right)\right\|-\left\|q_{1} A u_{1}\right\|\right| \leqq\left\|R_{2}\left(q_{1}\right)\right\|$ $\leqq r_{2} q_{1}^{2}$. Para $q_{1}$ suficientemente pequeno, temos $\left\|q_{1} A u_{1}+R_{2}\left(q_{1}\right)\right\| \geqq d_{0} q_{1}-r_{2} q_{1}^{2} \geqq \tilde{d}_{0} q_{1}$ para algum $\tilde{d}_{0}>0$. Logo

$$
\begin{gathered}
\left\|c_{1}-\frac{A u_{1}}{\left\|A u_{1}\right\|}\right\| \leqq \frac{r_{2} q_{1}}{\tilde{d}_{0}} \stackrel{q_{1} \rightarrow 0}{\longrightarrow} 0 \\
\left\|c_{2}\right\|=\frac{\left\|A_{i} R_{2}\left(q_{1}\right)\right\|}{\left\|q_{1} A u_{1}+R_{2}\left(q_{1}\right)\right\|} \leqq \frac{r_{2} q_{1}}{\tilde{\widetilde{d}}} \stackrel{q_{1} \rightarrow 0}{\longrightarrow} 0
\end{gathered}
$$

Concluímos que $X_{i}\left(x\left(q_{1}\right)\right) \stackrel{q_{1} \rightarrow 0}{\longrightarrow} \frac{A_{i} A u_{1}}{\left\|A u_{1}\right\|}$ e $N\left(x\left(q_{1}\right)\right) \stackrel{q_{1} \rightarrow 0}{\longrightarrow} \frac{A u_{1}}{\left\|A u_{1}\right\|}$ mas, em $W_{S_{0}}^{s}, q_{1} \rightarrow 0$ se e só se $x \rightarrow p_{c}$, completando a demonstração do lema.

Seja $m(t, x)$ o fluxo Hamiltoniano em $U$ gerado pela função dada em (5.1). Seja $n(t, y)$ o fluxo Hamiltoniano em $V$ associado à função (5.2). Usaremos a notação $m_{t}(x) \stackrel{\text { def }}{=} m(t, x)$ e $n_{t}(y)=n(t, y)$. 
Sabemos que o difeomorfismo $\varphi: V \rightarrow U$ conjuga os fluxos $m(t, x)$ e $n(t, y)$ onde estes estiverem definidos, ou seja,

$$
\varphi \circ n_{t}=m_{t} \circ \varphi
$$

Então-temos

$$
D_{y} \varphi D_{y} n_{t}=D_{x} m_{t} D_{y} \varphi
$$

Seja $x:[0, \infty) \rightarrow U$ uma solução de

$$
\dot{x}=J_{0} H_{x}(x)
$$

tal que $\lim _{t \rightarrow \infty} x(t)=p_{c}, x_{0} \in S_{0}$. Obviamente $x(t)=m(t, x(0))$. Sejam $X_{i}:[0, \infty) \rightarrow T S_{0}$, $i=1,2,3$ vetores ortonormais definidos em (4.2) tais que $\left\{X_{i}(t)\right\}_{i=1,2,3}$ geram $T_{x(t)} S_{0}$. Sabemos que a solução $y:[0, \infty) \rightarrow V$ dada por $y(t)=n_{t}\left(\varphi^{-1}(x(0))\right)$ é conjugada à solução $x(t)$ por $\varphi$ conforme (5.5) e corresponde a um ramo variedade estável de $0 \mathrm{em} V$. A soluçäo $y(t)$ satisfaz

$$
\dot{y}=J_{0} K_{y}(y)
$$

onde $K_{y}(y)$ é o vetor gradiente de $K$ no ponto $y$. Portanto, $y(t)=\left(q_{10} e^{-\bar{\alpha} t}, 0,0,0\right)$.

Podemos, da mesma forma trivializar $T V_{0}$, onde $V_{0} \stackrel{\text { def }}{=} V \backslash\{0\}$, obtendo sobre a solução $y(t)$ vetores ortonormais $\left\{Y_{i}(t)\right\}_{i=1,2,3}$ que geram $T_{y(t)} V_{0}$. Então, nas coordenadas $y=\left(q_{1}, q_{2}, p_{1}, p_{2}\right)$ em $V$, temos

$$
\begin{aligned}
& Y_{1}(t)=Y_{1}=(0,1,0,0) \\
& Y_{2}(t)=Y_{2}=(0,0,0,-1) \\
& Y_{3}(t)=Y_{3}=(-1,0,0,0)
\end{aligned}
$$

para todo $t \in[0, \infty)$.

Seja $v:[0, \infty) \rightarrow T S_{0}$ uma solução não nula do fluxo linearizado sobre $x(t)$

$$
\dot{v}=J_{0} H_{x x}(x(t)) v
$$




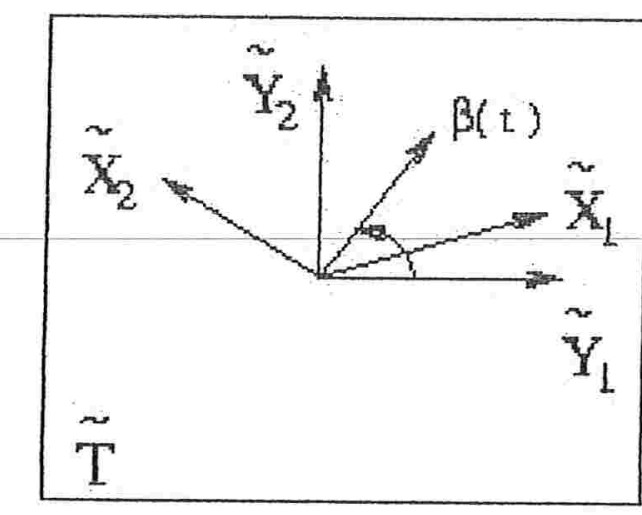

Figura ${ }^{2}$-1: Existem duas bases para $\tilde{T}: \tilde{X}$ e $\tilde{Y}$.

tal que $\alpha_{1}(t)^{2}+\alpha_{2}(t)^{2} \neq 0$ onde $v(t)=\alpha_{1}(t) X_{1}(t)+\alpha_{2}(t) X_{2}(t)+\alpha_{3}(t) X_{3}(t)$.

Queremos estimar o número de voltas que a projeção de $v(t)$ no plano gerado por $X_{1}(t)$ e $X_{2}(t)$ dá em torno da origem. Para isso, é suficiente estimarmos o número de voltas que o vetor $\left(\alpha_{1}(t), \alpha_{2}(t)\right) \in \mathbb{R}^{2}$ dá em torno da origem. Seja $\widetilde{X}_{i}(t) \stackrel{\text { def }}{=} D_{x} \varphi^{-1}(x(t)) X_{i}(t), i=1,2,3$ e seja $\tilde{T}(t)$ o plano gerado pelos vetores $\tilde{X}_{1}(t)$ e $\tilde{X}_{2}(t)$.

Como $\operatorname{span}\left\{X_{1}(t), X_{2}(t)\right\} \pitchfork X_{H}(x(t))$, então $\tilde{T}(t) \pitchfork X_{K}(y(t)) \Rightarrow \widetilde{T}(t) \pitchfork Y_{3}$. Portanto, podemos considerar o isomorfismo $\pi_{t}: \widetilde{T}(t) \rightarrow\left\{Y_{1}(t), Y_{2}(t)\right\}$ dado pela projeção ao longo de $Y_{3}(t)$, e definimos uma outra base em $\tilde{T}(t), \widetilde{Y} \stackrel{\text { def }}{=}\left\{\tilde{Y}_{1}(t), \tilde{Y}_{2}(t)\right\}$ dada por

$$
\tilde{Y}_{i}(t)=\pi_{t}^{-1}\left(Y_{i}\right), i=1,2
$$

Então temos

$$
\begin{aligned}
g(t) & =\beta^{1}(t) Y_{1}(t)+\beta^{2}(t) Y_{2}(t)+\beta^{3}(t) Y_{3}(t) \\
& =\alpha^{1}(t) \tilde{X}_{1}(t)+\alpha^{2}(t) \tilde{X}_{2}(t)+\alpha^{3}(t) \tilde{X}_{3}(t) \\
& =\beta^{1}(t) \tilde{Y}_{1}(t)+\beta^{2}(t) \tilde{Y}_{2}(t)+\alpha^{3}(t) \tilde{X}_{3}(t)
\end{aligned}
$$

Ver figura 5-1.

Logo

$$
\alpha^{1}(t) \tilde{X}_{1}(t)+\alpha^{2}(t) \tilde{X}_{2}(t)=\beta^{1}(t) \tilde{Y}_{1}(t)+\beta^{2}(t) \tilde{Y}_{2}(t)
$$


Sabemos que

$$
g(t)=D n_{t}(y(t)) g(0)
$$

Logo $g(t)$ é solução de

$$
\dot{g}=J_{0} K_{y y}(y(t)) g
$$

onde $K_{y y}(y(t))$ é a matriz Hessiana de $K$ no ponto $y(t)$. É fácil ver que

$$
K_{y y}(y(t))=\left(\begin{array}{llll}
0 & 0 & -\bar{\alpha} & 0 \\
0 & \bar{\omega} & 0 & 0 \\
-\bar{\alpha} & 0 & \gamma e^{-2 \bar{\alpha} t} & 0 \\
0 & 0 & 0 & \bar{\omega}
\end{array}\right)
$$

\section{Lema 5.2 Temos}

$$
\left(\begin{array}{l}
\beta^{1}(t) \\
\beta^{2}(t)
\end{array}\right)=\left(\begin{array}{cc}
\cos \bar{\omega} t & -\sin \bar{\omega} t \\
\sin \bar{\omega} t & \cos \bar{\omega} t
\end{array}\right)\left(\begin{array}{l}
\beta^{1}(0) \\
\beta^{2}(0)
\end{array}\right)
$$

para todo $t \geqq 0$.

Prova. Seja $\beta(t)=\left(\begin{array}{c}\beta^{1}(t) \\ \beta^{2}(t)\end{array}\right)$. Sabemos, por (4.6) que

$$
\dot{\beta}=-J S \beta
$$

onde, por (4.7),

$$
S=\left(\begin{array}{cc}
\left\langle K_{y y}(y(t)) Y_{1}(t), Y_{1}(t)\right\rangle & \left\langle K_{y y}(y(t)) Y_{1}(t), Y_{2}(t)\right\rangle \\
\left\langle K_{y y}(y(t)) Y_{1}(t), Y_{2}(t)\right\rangle & \left\langle K_{y y}(y(t)) Y_{2}(t), Y_{2}(t)\right\rangle
\end{array}\right)+\left\langle K_{y y} Y_{3}(t), Y_{3}(t)\right\rangle I
$$

Então, por (5.7) e (5.10), temos

$$
\begin{aligned}
& \left\langle K_{y y}(y(t)) Y_{1}(t), Y_{1}(t)\right\rangle=\bar{\omega} \\
& \left\langle K_{y y}(y(t)) Y_{1}(t), Y_{2}(t)\right\rangle=0 \\
& \left\langle K_{y y}(y(t)) Y_{2}(t), Y_{2}(t)\right\rangle=\bar{\omega}
\end{aligned}
$$




$$
\left\langle K_{y y} Y_{3}(t), Y_{3}(t)\right\rangle=0
$$

Logo

$$
S(t)=\left(\begin{array}{cc}
\bar{\omega} & 0 \\
0 & \bar{\omega}
\end{array}\right)
$$

e, portanto,

$$
\dot{\beta}=\left(\begin{array}{ll}
0 & -\bar{\omega} \\
\bar{\omega} & 0
\end{array}\right) \beta
$$

cuja solução é dada no enunciado do lema.

A solução $\left(\beta^{1}(t), \beta^{2}(t)\right)$ é, portanto, dada por

$$
\left(\begin{array}{l}
\beta^{1}(t) \\
\beta^{2}(t)
\end{array}\right)=\left(\begin{array}{cc}
\cos \bar{\omega} t & -\sin \bar{\omega} t \\
\sin \bar{\omega} t & \cos \bar{\omega} t
\end{array}\right)\left(\begin{array}{l}
\beta^{1}(0) \\
\beta^{2}(0)
\end{array}\right)
$$

que corresponde a uma órbita circular em torno da origem com velocidade angular positiva constante. A projeção de $g(t)$ em $\widetilde{T}(t)$ dá, portanto, infinitas voltas em torno da origem no referencial $\tilde{Y}$ no sentido anti-horário.

Pelo Lema 5.1, sabemos que os vetores $X_{i}(t)$ convergem quando $t \rightarrow \infty$. Logo $\widetilde{X}_{i}(t)$ e $\widetilde{Y}_{i}(t)$ também convergem. Como $\left(\beta^{1}(t), \beta^{2}(t)\right)$ gira infinitas vezes em torno da origem, então, por (5.9), o vetor $\beta^{1}(t) \tilde{Y}_{1}(t)+\beta^{2}(t) \widetilde{Y}_{2}(t)$ também deve girar infinitas vezes com relação ao referencial $\tilde{X}$. E, portanto, o vetor $\left(\alpha^{1}(t), \alpha^{2}(t)\right)$ também dá infinitas voltas em torno da origem.

Isso significa que o fluxo linearizado sobre um ramo da variedade estável de um sela-centro tende a um comportamento oscilatório quando projetado no plano gerado por pelos vetores $X_{1}$ e $X_{2}$.

Sabemos que as equações que determinam o índice de Conley-Zehnder de uma órbita periódica são

$$
\begin{aligned}
\dot{x} & =J_{0} H_{x}(x) \\
\left(\begin{array}{c}
\dot{\alpha}_{1} \\
\dot{\alpha}_{2}
\end{array}\right) & =-\left|H_{x}\right| J\left[\left(\begin{array}{ll}
\left\langle d N X_{1}, X_{1}\right\rangle & \left\langle d N X_{1}, X_{2}\right\rangle \\
\left\langle d N X_{1}, X_{2}\right\rangle & \left\langle d N X_{2}, X_{2}\right\rangle
\end{array}\right)+\left\langle d N X_{3}, X_{3}\right\rangle I\right]_{x(t)}\left(\begin{array}{c}
\alpha_{1} \\
\alpha_{2}
\end{array}\right)
\end{aligned}
$$


Lema 5.3 Se $S_{0}$ tem curvatura positiva, então dado $K \in \mathbb{N}$, existe uma vizinhança $W$ de $p_{c}$ em $S$ tal que se $P$ é uma órbita periódica que intersecta $W$, então $\mu_{C Z}(P)>K$.

Prova. Sabemos que sobre a solução $x_{s}(t),\left(\alpha^{1}(t), \alpha^{2}(t)\right)$ dá infinitas voltas em torno da origem no sentido anti-horário. Podemos então escolher $T_{0}$ tal que $\left(\alpha^{1}(t), \alpha^{2}(t)\right)$ dá $2\left(\left\lceil\frac{K}{2}\right\rceil+1\right)$ voltas em torno da origem no tempo $T_{0}$. Pelo Teorema do Fluxo Tubular Longo, existe uma vizinhança $W_{1}$ de $x_{s}(0)$ tal que qualquer órbita periódica que passa por $W_{2}$ tem período maior que $T_{0}$.

Por dependência contínua das soluções das equações (5.11) com relação às condiçốes iniciais, existe uma vizinhança $W_{0} \subset W_{1}$ de $x_{s}(0)$ tal que a solução $\alpha(t)$ sobre qualquer solução $x(t)$ que começa em $W_{0}$ dá pelo menos $\left\lceil\frac{K}{2}\right\rceil+1$ voltas em torno da origem no intervalo de tempo entre 0 e $T_{0}$.

Como $S_{0}$ tem curvatura positiva, sabemos, pelo Teorema 4.2, que o ângulo de $\left(\alpha^{1}(t), \alpha^{2}(t)\right)$ é crescente e portanto, após completar o período de uma órbita periódica $P$ que passa por $W_{0}$, o número de voltas de $\left(\alpha^{1}(t), \alpha^{2}(t)\right)$ será de pelo menos $\left\lceil\frac{K}{2}\right\rceil+1$. Pelas Proposições 4.1 e 4.2 , o índice de Conley-Zehnder de uma órbita periódica pode ser estimado pelo número de voltas, conforme sua definição geométrica e, portanto, $\mu_{C Z}(P)>K$. Pela forma normal do sela-centro, é fácil ver que podemos encontrar uma vizinhança $W$ de $p_{c}$ tal que se uma órbita periódica $P$ intersecta $W$ então $P$ intersecta $W_{0}$. Logo $\mu_{C Z}(P)>K$ para toda órbita periódica $P$ que intersecta $W$. 


\section{Capítulo 6}

\section{Seções Globais}

\subsection{Nivel de energia do sela-centro}

Seja $X_{H}$ o campo Hamiltoniano restrito ao nível de energia $S=H^{-1}(a)$ onde a é um valor regular de $H: \mathbb{R}^{4} \rightarrow \mathbb{R}$. Dizemos que $\sum \subset S$ é uma seção global do fluxo Hamiltoniano em $S$ se $\sum$ satisfaz as seguintes propriedades:

i) $\sum$ é um mergulho de uma superfície compacta;

ii) $\partial \sum$ é constituído por órbitas periódicas de $X_{H}$;

iii) $\sum^{\circ}$ (o interior de $\sum$ ) é transversal a $X_{H}$;

iv) A órbita de qualquer ponto que não esteja contido em $\partial \sum$ intersecta $\sum$ para frente e para trás no tempo;

O fluxo $\varphi(t, x)$ de $X_{H}$ induz, portanto, um difeomorfismo $\tilde{\varphi}: \sum^{\circ} \rightarrow \sum^{\circ}$ da seguinte forma: dado $x \in \sum^{\circ}$, definimos $\tilde{\varphi}(x)$ o primeiro ponto da órbita para frente de $x$ que intersecta $\sum^{\circ}$ novamente. A existência de uma seção global reduz, portanto, o estudo do fluxo num determinado nível de energia ao estudo de difeomorfismos em superfícies de dimensão 2.

Estamos interessados no caso em que $\sum$ é um disco, ou seja, existe um mergulho $u: D \rightarrow S$ tal que $u(D)=\sum$, onde $D=\left\{x \in \mathbb{R}^{2} \mid\|x\| \leqq 1\right\}$. $\partial \sum$ é, neste caso, uma órbita periódica do fluxo $\varphi(t, x)$. Também consideramos apenas o caso em que $S$ é difeomorfo a $S^{3}$.

Seja $T_{r}: \sum^{\circ} \rightarrow \mathbb{R}$ dada por $T_{r}(x)=\min \left\{t>0 \mid \varphi(t, x) \in \sum^{\circ}\right\}$, ou seja, $\varphi\left(T_{r}(x), x\right) \in \stackrel{\circ}{\sum}$ e $A$ $0<t<T_{r}(x)$ tal que $\varphi(t, x) \in \sum^{\circ}$. A função $T_{r}$ está bem definida, pois $\sum$ é seção global, e é $C^{1}$ em $\sum^{\circ}$ devido à regularidade de $\sum$ e do fluxo $\varphi(t, x)$. 
Para cada $x \in \sum^{\circ}$, seja $o_{1}(x) \stackrel{\text { def }}{=}\left\{\varphi(t, x) \in S \mid 0 \leqq t<T_{r}(x)\right\}$. Então $\partial \sum \cup\left\{\cup o_{1}(x)\right\}=S$. $x \in \sum^{\circ}$

Seja $\pi_{\Sigma}: S \backslash \partial \sum \rightarrow \sum^{\circ}$ onde $\pi_{\Sigma}(x)=z \in \sum^{\circ}$ se $x \in o_{1}(z)$. Seja $\Delta T_{c}: S \backslash \partial \sum \rightarrow \mathbb{R}^{+}$dada por $\Delta T_{c}(x)=t \geqq 0$ onde $\varphi\left(t, \pi_{\sum}(x)\right)=x$. Obviamente $\left.\Delta T_{c}\right|_{\Sigma}=0$ e $0 \leqq \Delta T_{c}(x)<T_{r}\left(\pi_{\sum}(x)\right)$ para todo $x \in S \backslash \partial \sum$.

Proposição 6.1 Seja $\sum$ uma seção global para o fluxo $\varphi(t, x)$. Seja $\Delta T_{1}: \sum \rightarrow \mathbb{R}^{+}$uma função $C^{1}$ tal que

$$
\begin{array}{r}
0 \leqq \Delta T_{1}(x)<T_{r}(x) \text { se } x \in \sum^{\circ} \\
\Delta T_{1}(x)=0 \text { numa vizinhança de } \partial \sum \text { em } \sum
\end{array}
$$

Entâo $\tilde{\sum}=\left\{\varphi\left(\Delta T_{1}(x), x\right), x \in \sum\right\}$ é uma seção global para $\varphi(t, x)$.

Prova. Seja $\widetilde{u}: D \rightarrow S$ dada por

$$
\tilde{u}(z)=\varphi\left(\Delta T_{1}(u(z)), u(z)\right)
$$

onde $u: D \rightarrow S$ é um mergulho tal que $u(D)=\sum$. Então $\tilde{u}(D)=\tilde{\sum}$ e mostraremos que $\tilde{u}$ também é um mergulho. Como $\sum$ é uma seção transversal ao campo $X_{H}$, então $T_{x} S=$ $T_{x} \sum \oplus X_{H}(x)$ e, como $\varphi(t, \cdot)$ é um difeomorfismo,

$$
T_{\widetilde{u}(z)} S=D_{x} \varphi\left(\Delta T_{1}(u(z)), u(z)\right) T_{u(z)} \sum \oplus X_{H}(\tilde{u}(z))
$$

Por outro lado, temos

$$
\begin{aligned}
D_{z} \tilde{u} & =D_{x} \varphi\left(\Delta T_{1}(u(z)), u(z)\right) D_{z} u+\frac{\partial \varphi\left(\Delta T_{1}(u(z)), u(z)\right)}{\partial t} D_{x} \Delta T_{1}(u(z)) D_{z} u \\
& =D_{x} \varphi\left(\Delta T_{1}(u(z)), u(z)\right) D_{z} u+X_{H}(\tilde{u}(z)) D_{x} \Delta T_{1}(u(z)) D_{z} u
\end{aligned}
$$

Devemos mostrar que a transformação linear $D_{z} \widetilde{u}(z)$ é injetora. Como u é um mergulho, $D_{z} u(z) T_{z} D=T_{u(z)} \sum$. Seja $\left\{w_{1}, w_{2}\right\}$ uma base de $T_{u(z)} \sum$. Sejam $m_{i}, i=1,2$, os vetores em 
$T_{\dddot{u}(z)} \sum$, dados por

$$
m_{i}=D_{x} \varphi\left(\Delta T_{1}(u(z)), u(z)\right) w_{i}+X_{H}(\tilde{u}(z)) D_{x} \Delta T_{1}(u(z)) w_{i}
$$

Por (6.1) e (6.2), os vetores $m_{1}$ e $m_{2}$ são linearmente independentes e, portanto, $\tilde{u}$ é uma imersão. Pelo mesmo motivo, a reta $\mathbb{R} X_{H}\left(\varphi\left(\Delta T_{1}\left(u\left(z_{0}\right)\right), u\left(z_{0}\right)\right)\right)$ não está contida em $T_{\tilde{u}(z)} \sum$ . Logo $\sum^{\varkappa}$ é transversal ao campo $X_{H}$.

Obviamente $\tilde{u}$ é contínua e injetora e sua inversa é dada por

$$
\widetilde{u}^{-1}(z) \stackrel{\text { def }}{=} \varphi\left(-\Delta T_{1}\left(\left(\pi_{\Sigma}(z)\right)\right), z\right)
$$

Como $\Delta T_{1}(x)<T_{r}(x)$ para todo $x \in \sum$, podemos encontrar uma vizinhança $U_{x}$ de $x \in \tilde{\Sigma}$ em $S$ tal que para cada $y \in U_{x} \cap \sum$, temos $U_{x} \cap \tilde{\sum} \cap_{1}\left(\pi_{\sum}(y)\right)=\{y\}$. Isso implica que $\tilde{u}$ é um homeomorfismo de $D$ sobre $\tilde{u}(D)$, considerando-se em $\tilde{u}(D)$ a topologia induzida por $S$.

Temos ainda $\partial \sum=\partial \sum$ pois $\Delta T_{1}$ é zero numa vizinhança de $\partial \sum$. Obviamente a órbita

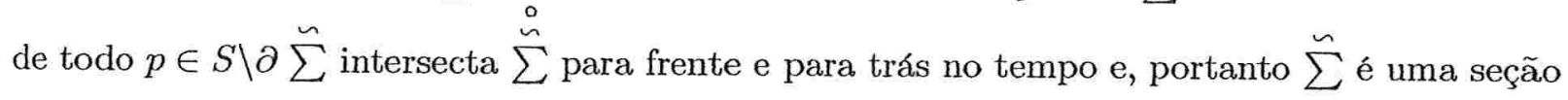
global para o fluxo $\varphi(t, z)$ em $S$.

Sabemos que hipersuperfícies difeomorfas a $S^{3}$ e estritamente convexas possuem seções globais. Mais precisamente, temos o seguinte Teorema de Hofer-Zehnder-Wysocki[HZW]

Teorema 6.1 Seja $S=H^{-1}(c) \subset \mathbb{R}^{4}$ uma hipersuperfície regular, compacta e estritamente convexa. Então existe uma órbita periódica $P$ do fluxo Hamiltoniano de $X_{H}$ com as seguintes propriedades:

(i) $P=\partial \sum$ é o bordo de um disco compacto $\sum$ mergulhado em $S$;

(ii) $\sum^{\circ}=\sum \backslash \partial \sum$ é transversal a $X_{H}$;

(iii) $\sum$ é uma secão global para $X_{H}$;

(iv) $\mu_{C Z}(P)=3$ onde $\mu_{C Z}(P)$ é o índice de Conley-Zehnder da órbita periódica $P$, considerando seu período mínimo;

(v) $P$ não forma um nó em $S$ e $s l(P)$, o self-linking number de $P$, é igual a -1 .

Além disso, o fluxo gerado por $X_{H}$ possui 2 ou $\infty$ órbitas periódicas em $S$. 
Para maiores detalhes sobre a definição do self-linking number de uma órbita periódica, ver [HZW].

Um importante resultado de [HZW] é que se $P \subset S$ é uma órbita periódica com índice de Conley-Zehnder igual a 3, self-linhling number igual a -1 e tal que $P$ não forma um nó em $S$ então $P$ é o bordo de uma seção global em $S$.

Consideraremos hipersuperfícies em $\mathbb{R}^{4}$ que não necessariamente sejam regulares, ou mais precisamente, que deixam de ser regulares em um único ponto $p_{c}$, correspondente à um ponto de equilíbrio do tipo sela-centro. Queremos encontrar condições para existência de seções globais em hipersuperfícies não-regulares.

Seja $H: \mathbb{R}^{4} \rightarrow \mathbb{R}$ uma função Hamiltoniana real-analítica e $S \subset H^{-1}(c)$ uma hipersuperfície homeomorfa a $S^{3}$ com as seguintes propriedades:

i) $S$ é regular em todos os pontos exceto em $p_{c}$;

ii) $p_{c}$ é um ponto de equilíbrio do tipo sela-centro do fluxo Hamiltoniano associado a $H$.

iii) Para todo $q \in S, q \neq p_{c}$, o Hessiano de $H$ restrito a $T_{q} S$ é definido;

(iv) Existe um plano suporte $H_{p_{c}}$ de $B_{S}$, o interior de $S$, passando por $p_{c}$;

Lembramos que $p_{c}$ possui variedades estável e instável unidimensionais e, portanto, devemos adaptar o conceito de seção global. Chamamos $\sum$ de seção global para $S$ quando $p_{c} \notin \sum \mathrm{e}$ $\sum$ satisfaz todas as propriedades de seção global exceto que existem $x_{s}$ e $x_{u}$ pontos em $\sum$ tais que $\lim _{t \rightarrow \infty} \varphi_{t}\left(x_{s}\right)=p_{c}$ e $\lim _{t \rightarrow \infty} \varphi_{-t}\left(x_{u}\right)=p_{c}$, respectivamente, com a propriedade que tais semi-órbitas não passam por $\sum$ para $t>0$. Se definirmos $\tilde{\varphi}: \sum \rightarrow \sum$ por: dado $x \in \sum, \tilde{\varphi}(x)$ é o primeiro ponto da órbita para frente de $x$ que intersecta $\sum$ novamente se $x \neq x_{s}, \tilde{\varphi}\left(x_{e}\right)=x_{u}$ e $\tilde{\varphi}^{-1}\left(x_{u}\right)=x_{s}$, obtemos um homeomorfismo do disco $\sum$.

Seja $U_{\varepsilon}$ uma vizinhança de $p_{c}$ contida em $B_{\varepsilon}\left(p_{c}\right)$ onde vale o Teorema de Moser tal que se $P$ é uma órbita periódica do fluxo $X_{H}$ em $S$ que intersecta $U_{\varepsilon}$, então $\mu_{C Z}(P)>3$. A existência dessa vizinhança é garantida pelo Teorema 4.3. Seja $S_{U_{\varepsilon}} \stackrel{\text { def }}{=} S \backslash U_{\varepsilon}$. Utilizando o Teorema 2.2 , podemos regularizar $S_{U_{\varepsilon}}$ de modo a obter uma hipersuperfície $\widetilde{S}_{\varepsilon} \supset S_{U_{\varepsilon}}$ difeomorfa a $S^{3}$ e estritamente convexa. Aplicando o Teorema 6.1 garantimos que existe em $\widetilde{S}_{\varepsilon}$ uma órbita periódica $P$ que é bordo de uma seção global $\sum_{\varepsilon}$. Além disso, sabemos que $\mu_{C Z}(P)=3$. Seja $K_{\varepsilon} \subset \widetilde{S}$ o compacto definido por $\bar{K}_{\varepsilon} \stackrel{\text { def }}{=}\left(\tilde{S}_{\varepsilon} \backslash S_{U_{\varepsilon}}\right) \cup \partial S_{U_{\varepsilon}}$. Se $P \cap K_{\varepsilon} \neq \emptyset$ e $P \cap S_{U_{\varepsilon}} \neq \emptyset$, então pelo Lema 5.3, $\mu_{C Z}(P)>3$, portanto, $P \subset S_{U_{\varepsilon}}$ ou $P \subset K_{\varepsilon}$. Mas pela Proposição $4.5, P \nsubseteq K_{\varepsilon}$. 


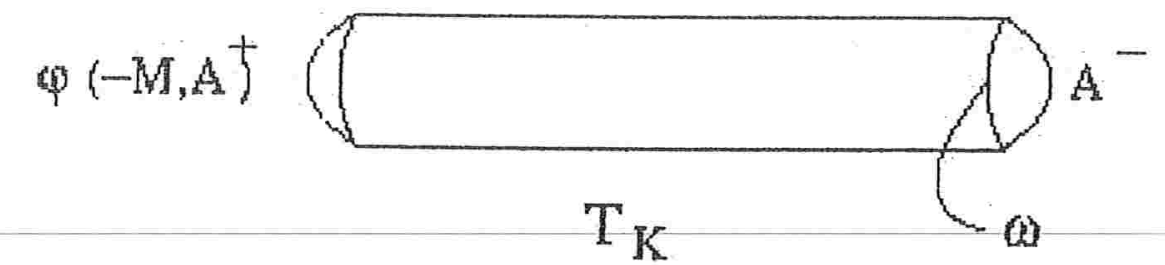

Figura 6-1: Representação esqumática do cilindro sólido $T_{K}$.

Logo $P \subset S_{U_{\varepsilon}}$. Fazendo $\varepsilon \rightarrow 0$ temos que $P$ também é bordo de uma seção global para $\widetilde{S}_{\varepsilon}$ pois o índice de Conley-Zehnder e o self-linking number de $P$ não se alteram com modificações em $S$ longe de $P$. Portanto, consideramos, para todo $\varepsilon>0$ a mesma órbita periódica $P$ que é bordo de uma seção global para $\widetilde{S}_{\varepsilon}$. Omitiremos o índice $\varepsilon$ para facilitar de notação.

O conjunto $K \subset \tilde{S}$ é um compacto difeomorfo a $B^{3}$ com as seguintes propriedades:

(i) $\partial \sum \cap K=\emptyset$;

(ii) $\partial K=A^{+} \cup A^{-} \cup \omega$, onde $A^{+}$é um disco aberto tal que se $x \in A^{+}$então $\varphi(t, x) \in K$ para todo $t>0$ suficientemente pequeno e $\varphi(t, x) \notin K$ para todo $t<0$ suficientemente pequeno. Analogamente $A^{-}$é um disco aberto tal que se $x \in A^{-}$então $\varphi(t, x) \notin K$ para todo $t>0$ suficientemente pequeno e $\varphi(t, x) \in K$ para todo $t<0$ suficientemente pequeno. O conjunto $\omega$ é difeomorfo a $S^{1}$ e é formado pelos pontos $x$ cuja órbita tangencia $K$ e para todo tempo $t \neq 0$ suficientemente pequeno $\varphi(t, x) \notin K$. Além disso $\omega=\partial A^{+}=\partial A^{-}$.

(iii) Existe um vetor $N \in \mathbb{R}^{4}$ tal que $\langle N(x), N\rangle \neq 0$ para todo $x \in K$, onde $N(x)$ é um vetor normal a $K$ em $x$.

Associado ao conjunto $K$ vamos construir um outro conjunto $T_{K} \subset S$ da seguinte forma: consideremos uma função Hamiltoniana $H: \mathbb{R}^{4} \rightarrow \mathbb{R}$ tal que $\widetilde{S}$ é um nível regular de $H$ e o campo Hamiltoniano $X_{H}$ satisfaz $\lambda_{0}\left(X_{H}\right)=1$ (ver definição de $\lambda_{0}$ em (8.1)).

Seja $T_{K}=K \cup \underset{0 \leqq t \leqq M}{U} \varphi\left(-t, A^{+} \cup \omega\right)$ onde $\varphi(t, x)$ é o fluxo do campo $X_{H}$ e $M>0$ é tal que $\varphi\left(-t, A^{+} \cup \omega\right) \cap\left\{A^{-} \cup \omega\right\}=\emptyset$ para $0<t \leqq M$.

$\mathrm{O}$ conjunto $T_{K}$ é um cilindro sólido cujo bordo é dado por $\partial T_{K}=\partial A^{-} \cup \underset{0 \leqq t \leqq M}{\cup} \varphi(-t, \omega) \cup$ $\varphi\left(-M, A^{+}\right)$. Ver figura 6-1.

Suponhamos que $\sum$ intersecta $\partial T_{K}$ transversalmente. Então $\sum \cap \partial T_{K}=\underset{i=1 . . r}{\cup} C_{i}$ onde cada $C_{i}$ é uma curva fechada simples. Consideremos o conjunto $I_{\Sigma}=\sum \cap T_{K}$. Então $\partial I_{K}$ 


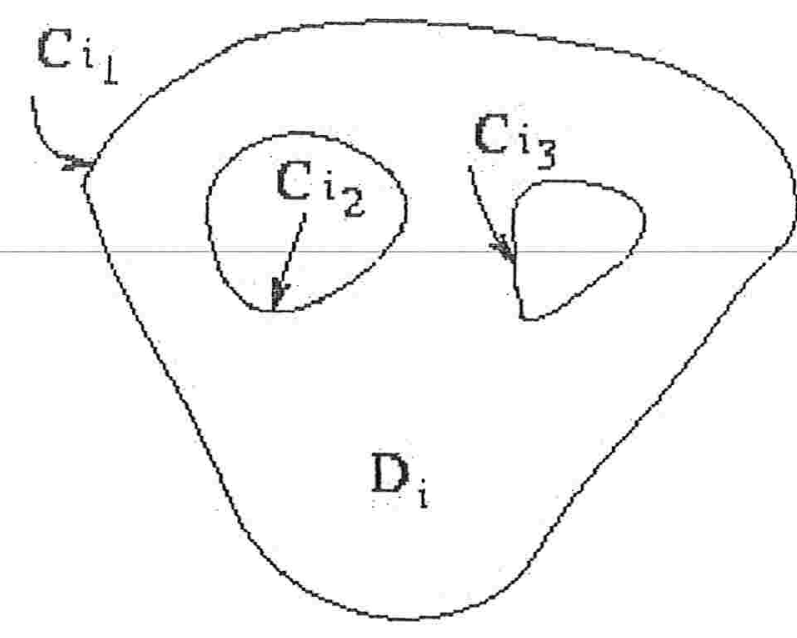

Figura 6-2: $\mathrm{O}$ bordo de $D_{i}$ é formado por um número finito de curvas fechadas simples.

$=\sum n \partial T_{K}=\bigcup_{i=1 . . r} C_{i}$ pois $\partial \sum n T_{K}=\emptyset$. O conjunto $I_{\sum}$ é formado por $s$ componentes conexas, denotadas por $D_{j}$. O bordo de $D_{j}$ é um conjunto finito de $C_{j_{i}}$ 's. Ver figura 6-2.

Consideremos agora a seguinte hipótese

$$
\text { (H1) } D_{j} \cap \partial A^{-}=\emptyset \text { ou } D_{j} \cap \varphi\left(-M, \partial A^{+}\right)=\emptyset \text { para cada } j \in\{1,2, . ., s\}
$$

\section{Então temos}

Teorema 6.2 Suponhamos que para algum $\varepsilon>0$ vale a hipótese (H1). Então existe uma seção global $\sum_{0}$ de $\tilde{S}$ tal que $\sum_{0} \cap K=\emptyset$ e $\partial \sum_{0}=\partial \sum=P$. Além disso, $\sum_{0}$ também é seçấo global para $S$ e se não existe órbita homoclínica a $p_{c}$, então o fluxo Hamiltoniano em $S$ possui 2 ou $\infty$ órbitas periódicas.

Prova. Movimentaremos $\sum$ numa vizinhança de $D_{j}$ para eliminarmos todas as interseções de $\sum \operatorname{com} K$. Suponhamos que $D_{j} \cap \varphi\left(-M, \partial A^{+}\right)=\emptyset$. Queremos movimentar $D_{j}$ para frente pelo fluxo para tirá-lo de $T_{K}$. Mas $D_{j}$ pode estar obstruída por outra componente $D_{i}$, ou seja, não podemos movimentar $D_{j}$ até tirarmos de $T_{K}$ sem intersectarmos $D_{i}$ durante o movimento. Ver figura 6-3.

Como $\sum$ é transversal ao fluxo e $T_{K}$ é uma caixa de fluxo, se $D_{i}$ obstrui $D_{l}$ para o movimento 


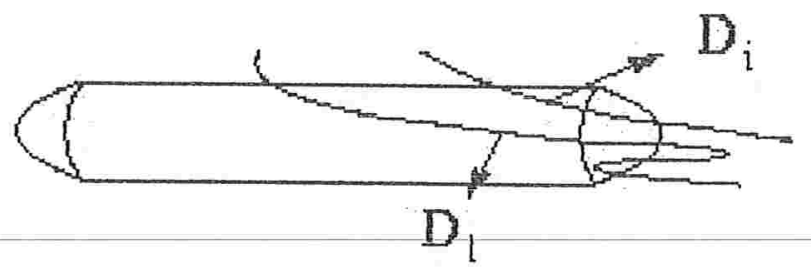

Figura 6-3: Uma componente $D_{i}$ pode obstruir a movimentação para frente no tem= po de $D_{l}$.

para frente, então $D_{l}$ não pode obstruir $D_{i}$ para o movimento para frente. Como temos um número finito de componentes conexas, então existe pelo menos uma componente conexa $D_{j}$ que está desobstruída para o movimento para frente, ou seja, podemos usar o fluxo para tirarmos $D_{j}$ de $T_{K}$ sem termos problemas com novas intersecções.

Seja $\tilde{h}_{j}: D_{j} \rightarrow \mathbb{R}^{+}$dada por $\varphi\left(x, h_{j}(x)\right) \in A^{-}$. A função $\tilde{h}_{j}$ está bem definida e é diferenciável. Além disso, como o bordo de $D_{j}$ é formado por um número finito de curvas regulares, fechadas e simples, então dada uma vizinhança $U_{j}$ de $D_{j}$ em $\sum$, podemos estender $\widetilde{h}_{j}$ obtendose uma função diferenciável $h_{j}: \sum \rightarrow \mathbb{R}^{+}$tal que $h_{j}(x)=\widetilde{h}_{j}(x)$ se $x \in D_{j}$ e $h_{j}(x)=0$ se $x \in \sum \backslash U_{j}$. Seja $\phi: \sum \rightarrow \mathbb{R}^{+}$uma função $C^{\infty}$ que satisfaz $\phi(x)=1$ se $x \in D_{j}, 0 \leqq \phi(x)<1$ se $x \in U_{j} \backslash D_{j}$ e $\phi(x)=0$ se $x \in \sum \backslash U_{j}$. Escolhendo-se a vizinhança $U_{j}$ suficientemente pequena, $n$ suficientemente grande e $\varepsilon_{j}>0$ suficientemente pequeno, temos que $\varphi\left(\phi^{n}\left(h_{j}(x)+\varepsilon_{j}\right), x\right) \notin T_{K}$ para todo $x \in U_{j}$. A função $h_{j}$ satisfaz as hipóteses da Proposição 6.1, e, portanto, o conjunto $\Sigma^{\prime} \stackrel{\text { def }}{=}\left\{\varphi\left(\phi^{n}\left(h_{j}(x)+\varepsilon_{j}\right), x\right), x \in \sum\right\}$ é uma seção global para o fluxo.

Além disso, como movimentamos apenas $D_{j}$ o conjunto $I_{\Sigma^{\prime}}$ possui uma componente conexa a menos do que $I_{\Sigma}$. Ver figura 6-4.

Como temos um número finito de componentes conexas, podemos continuar o mesmo processo de movimentação destas até obtermos uma seção global $\sum_{0}$ para o fluxo que não intersecte $K$. Usamos o mesmo processo para movimentarmos para trás no tempo as componentes conexas que intersectam $\varphi\left(-M, \partial A^{+}\right)$.

Basta agora provar que $\sum_{0}$ é superfície de seção global para o fluxo Hamiltoniano em $S$. Seja $\tilde{H}: \mathbb{R}^{4} \rightarrow \mathbb{R}$ uma função $C^{k \geqq 2}$ tal que $\widetilde{S}=\widetilde{H}^{-1}(c)$ para algum valor regular $c$. Seja $\Lambda$ o conjunto dos pontos $x \in \sum_{0}$ tal que a órbita para frente de $x$, pelo fluxo de $X_{\tilde{H}}$, passa por $K$ antes de 

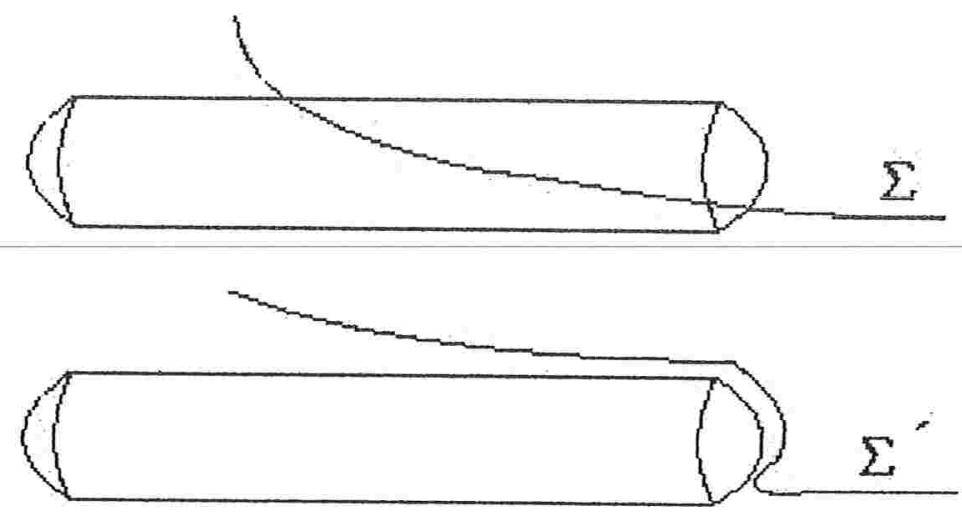

Figura 6-4: Obtemos uma nova seção global depois de movimentarmos uma componente $D_{i}$.

retornar a $\sum_{0}$ novamente. Então $\Lambda$ é um disco topológico em $\sum$ e definimos o homeomorfismo $\psi: \sum_{0} \rightarrow \sum_{0}$ da seguinte forma: Se $x \notin \Lambda$ então $\psi(x)=\tilde{\psi}(x)$ onde $\tilde{\psi}: \sum_{0} \rightarrow \sum_{0}$ é a aplicação de retorno associado ao fluxo em $\tilde{S}$. Se $x \in \Lambda$, então seja $y_{x}$ o primeiro ponto contido em $\partial K$ da órbita para frente de $x$ pelo fluxo de $X_{\tilde{H}}$ (note que podemos também usar o fluxo de $X_{H}$ ). Seja $z_{x}$ o ponto de saída de $y_{x}$ de $K$ pelo fluxo de $X_{H}$. Se $y_{x}$ é o ponto da variedade estável de $p_{c}$, cuja órbita para frente por $X_{H}$ fica sempre em $U$, então definimos $z_{x}$ como sendo o único ponto da variedade instável de $p_{c}$ no bordo de $K$ cuja órbita para trás por $X_{H}$ converge para $p_{c}$ e está sempre contida em $K$. Finalmente, definimos $\psi(x)$ como o primeiro ponto da órbita para frente de $z_{x}$, por $X_{\breve{H}}$ (ou $\left.X_{H}\right)$, que passa por $\sum_{0}$. Definindo de forma análoga $\psi^{-1}$, trocando o papel da variedade estável com o da variedade instável de $p_{c}$, temos que $\varphi$ é um homeomorfismo de $\sum_{0}$.

Para provarmos a existência de órbitas periódicas em $S$ quando não existe órbita homoclínica a $p_{c}$, usaremos a mesma idéia apresentada em [HZW2]. Sabemos que $\psi$ preserva área (a forma simplética $d \lambda_{0}$ restrita a $\sum_{0}$ ) e, portanto, existe um ponto fixo $\bar{p}$ de $\psi$. Este ponto fixo corresponde a um órbita periódica em $S$. Consideramos agora a restrição $\bar{\psi}$ do homeomorfismo $\psi$ ao anel $\sum_{0} \backslash\{\bar{p}\}$. Por um resultado de J. Franks $([\mathrm{F}])$, como $\bar{\psi}$ preserva área, então $\bar{\psi}$ tem 0 ou $\infty$ pontos periódicos. Cada ponto periódico de $\bar{\psi}$ também corresponde a uma órbita periódica em $S$. Portanto $S$ possui 2 ou $\infty$ órbitas periódicas. 


\subsection{Niveis de energia acima do sela-centro}

Fixaremos agora a função Hamiltoniana real-analítica $H: \mathbb{R}^{4} \rightarrow \mathbb{R}$ com as propriedades anteriores, ou seja, existe um ponto de equilíbrio $p_{c}$ do tipo sela-centro do sistema Hamiltoniano associado, que está contido numa hipersuperfície $S \subset H^{-1}(0)$, homeomorfa a $S^{3}$ e estritamente convexa. Suponhamos, sem perda de generalidade, que o Hessiano de $H$ é positivo-definido em $T S \backslash\left\{p_{c}\right\}$.

Para todo $E<0$ suficientemente próximo de 0 , existe uma hipersuperfície regular $S_{E} \subset$ $H^{-1}(E)$ difeomorfa a $S^{3}$ que converge para $S$ quando $E \rightarrow 0$ na topologia $C^{0}$ e na topologia $C^{\infty}$ fora de uma vizinhança de $p_{c}$. Em $S_{E}$ vale o Teorema 4.4, ou seja, existe seção global em $S_{E}$ para o fluxo Hamiltoniano e este pode ser estudado por um difeomorfismo que preserva área no disco aberto. Não consideraremos, portanto, tais níveis de energia.

Para $E>0$, os níveis de energia que estão próximos de $S$ podem não ser compactos e há órbitas que escapam de qualquer vizinhança de $S$, por menor que seja o valor de $E$. $O$ comportamento de tais órbitas pode ser melhor visualizado pela forma normal de Moser dada pela função Hamiltoniana (3.1). Suponhamos que $S$ corresponda a $\left\{K=0, q_{1} \geqq 0, p_{1} \geqq 0\right\}$. Então a hipersuperfície $S_{E}$ contém pontos que em $V$ correspondem a $\left\{q_{1}>0, p_{1}<0, I_{2}<\right.$ $\left.I^{c}(E)\right\}$ e a órbita para frente de tais pontos escapam de uma vizinhança de $S$.

No entanto, sabemos que, para $\varepsilon>0$ fixo, $S_{E} \backslash B_{\varepsilon}\left(p_{c}\right)$ possui uma componente $S_{E}^{-}$que converge para $S$ na topologia $C^{\infty}$ quando $E \rightarrow 0$. Portanto, para todo $E>0$ suficientemente pequeno, o Hessiano de $H$ será positivo-definido em $x \in S_{E}^{-}$. Ver figura 6-5.

Queremos estender $S_{E}^{-}$a um ovalóide utilizando o Teorema 2.2. Para isso, precisamos mostrar que o hiperplano $H_{p}$ que passa por um ponto $p \in \partial S_{E}^{-}$intersecta $\partial S_{E}^{-}$apenas no ponto $x$. Mostraremos por contradição. Se isso não ocorre, então existe uma seqüência $E_{n} \rightarrow 0$ e $p_{n} \in \partial S_{E_{n}}^{-}$tal que $H_{p_{n}} \cap \partial S_{E_{n}}^{-} \supset\left\{y_{n}\right\}, y_{n} \neq p_{n}$. Por compacidade e continuidade, temos $p_{n} \rightarrow p_{0} \in S, y_{n} \rightarrow y_{0} \in S, H_{p_{n}} \rightarrow H_{p_{0}}$ e $H_{y_{n}} \rightarrow H_{y_{0}}$ quando $n \rightarrow \infty$. Logo $y_{0} \in H_{x_{0}}$. Mas se $x_{0} \neq y_{0}$ então temos uma contradição, pois $S$ é estritamente convexa. Se $x_{0}=y_{0}$ então para $n$ suficientemente grande $p_{n}$ e $y_{n}$ estão contidos na vizinhança dada pelo Lema 2.1, onde sabemos que $S_{E_{n}}$ é localmente estritamente convexa, ou seja, chegamos a uma nova contradição.

Portanto, existe $E_{0}>0$ tal que para todo $0<E<E_{0}$ existe uma hipersuperfície $S_{E}^{-} \subset$ $H^{-1}(E)$ que pode ser estendida, pelo Teorema 2.2 , a um ovalóide, que denotaremos por $\tilde{S}_{E}$. 

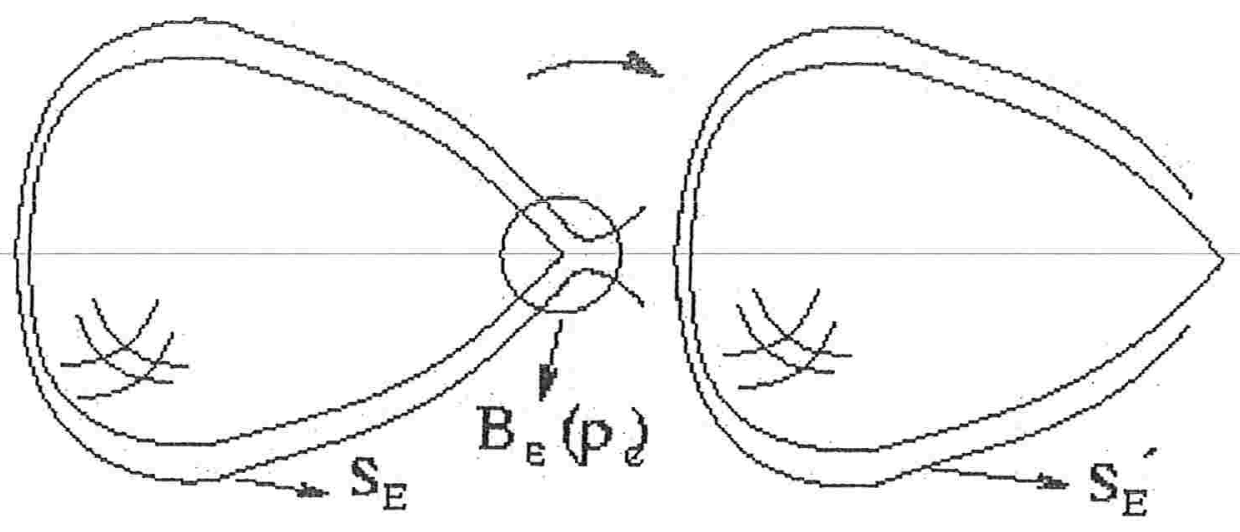

Figura 6-5: Existe uma componente convexa $S_{E}^{\prime} \subset S_{E}$ que pode ser estendida a um ovalóide.

Podemos também escolher $\varepsilon>0$ de modo que toda órbita periódica em $\widetilde{S}_{E}$ com índice de Conley-Zehnder igual a 3 esteja contida em $S_{E}^{-}$. Basta seguir a mesma idéia do Lema 5.3. Pelo Teorema 6.1, existe uma órbita periódica $P_{E} \subset S_{E}^{-}$que é bordo de uma seção global $\sum_{E}$ para o fluxo em $\widetilde{S}_{E}$. Omitiremos o índice $E$ para facilitar a notação.

Da mesma forma que fizemos no nível de energia 0 , para cada $E>0$, seja $K$ um compacto com as seguintes propriedades:

(i) $o=\partial \sum \cap K=\emptyset$;

(ii) $\partial K=A^{+} \cup A^{-} \cup \omega$, onde $A^{+}$é um disco aberto tal que se $x \in A^{+}$então $\varphi(t, x) \in K$ para todo $t>0$ suficientemente pequeno e $\varphi(t, x) \notin K$ para todo $t<0$ suficientemente pequeno. Analogamente $A^{-}$é um disco aberto tal que se $x \in A^{-}$então $\varphi(t, x) \notin K$ para todo $t>0$ suficientemente pequeno e $\varphi(t, x) \in K$ para todo $t<0$ suficientemente pequeno. O conjunto $\omega$ é difeomorfo a $S^{1}$ e é formado pelos pontos $x$ cuja órbita tangencia $K$ e para todo tempo $t \neq 0$ suficientemente pequeno $\varphi(t, x) \notin K$. Além disso $\omega=\partial A^{+}=\partial A^{-}$.

(iii) O conjunto $K$ é tal que $\widetilde{S}_{E} \cap K^{c} \subset S_{E}^{-}$.

Associado ao conjunto $K$, seja $T_{K} \subset S_{E}$ o cilindro sólido construído conforme anteriormente.

Podemos supor que $\sum$ intersecta $\partial T_{K}$ transversalmente. A transversalidade é garantida pelo Teorema de Sard (ver [Mi]), considerando-se a folhação de seções globais numa vizinhança de $\sum$. Então, como anteriormente $\sum n \partial T_{K}=\underset{i=1 . . r}{\cup} C_{i}$ onde cada $C_{i}$ é uma curva fechada simples. Seja $I_{\Sigma}=\sum \cap T_{K}$. Então $\partial I_{K}=\sum \cap \partial T_{K}$ pois $\partial \sum \cap T_{K}=\emptyset$. O conjunto $I_{\Sigma}$ é formado por 
$s$ componentes conexas, denotadas por $D_{j}, j=1 . . s$. O bordo de $D_{j}$ é um conjunto finito de $C_{i}^{\prime}$ s.

Consideremos a segunite hipótese

(H2) $D_{j} \cap \dot{A}^{-}=\emptyset$ ou $D_{j} \cap \varphi\left(-M, A^{+}\right)=\emptyset$ para cada $j \in\{1,2, . ., s\}$.

Teorema 6.3 Suponhamos que para um dado nível de energia $E>0$, vale a hipótese (H₹). Então existe uma seçấo global $\tilde{\sum}$ de $\widetilde{S}_{E}$ tal que $\sum^{\tilde{n}} \cap K=\emptyset$ e $\partial \tilde{\Sigma}=\partial \sum=P$. Além disso, se as variedades estável e instável de $o_{E}$ não coincidem, então existem infinitas órbitas homoclínicas a $o_{E}$, a órbita periódica no nível de energia $E$ contida, na variedade central de $p_{c}$.

Prova. A construção de $\sum^{\complement}$ é exatamente a mesma feita na demonstração do Teorema 6.1. Seja $\tilde{\varphi}: \check{\sum} \rightarrow \tilde{\sum}$ o difeomorfismo que representa o fluxo em $\breve{S}_{E}$. Consideremos agora os pontos $x \in \tilde{\sum}$ tais que a órbita para frente de $x$ intersecta $K$ antes de retornar a $\tilde{\sum}$. Chamemos tal conjunto de $\Lambda$. Então $\Lambda$ é um disco topológico fechado contido em $S_{E}^{-}$. Consideremos agora o fluxo Hamiltoniano $\varphi_{H}$ dado pela função $H$. Sabemos que existe uma curva de Jordan $c_{E}$ em $\tilde{\sum}$ que corresponde a uma curva não homotopicamente trivial da variedade estável de $o_{E}$. Pela construção do compacto $K$, temos que $c_{E} \subset \Lambda$. Seja $d_{E}$ o disco fechado em $\sum$ cujo bordo é $c_{E}$. Consideremos agora a aplicação $\psi: \sum^{n} \backslash d_{E} \rightarrow \sum^{\varkappa}$ definida da seguinte forma: Se $x \notin \Lambda$ então $\psi(x)=\tilde{\varphi}(x)$. Observe que esse segmento de órbita é o mesmo tanto para $\widetilde{S}_{E}$ quanto para $S_{E}$. Se $x \in \Lambda \backslash d_{e}$, então seja $y_{x}$ o primeiro ponto contido em $\partial K$ da órbita para frente de $x$ pelo fluxo $\varphi_{H}$. Seja $z_{x}$ o ponto de saída de $y_{x}$ de $K$ dado também pelo fluxo $\varphi_{H}$. Observe agora que a existência de $z_{x}$ é facilmente verificada pela forma normal de Moser. Finalmente, seja $\psi(x)$ o primeiro ponto da órbita para frente de $z_{x}$ a intersectar $\sum^{\varkappa}$.

A aplicação $\psi$ não pode ser estendida de forma contínua em $c_{E}$ pois, há um twist que tende a infinito para pontos que se aproximam de $c_{E}$. Esse twist decorre da estrutura do sela-centro e localmente pode ser entendido pela aplicação (3.3).

Da mesma forma, existe uma curva de Jordan $j_{E}$ em $\sum$ que corresponde a uma curva não homotopicamente trivial da variedade insvável de $o_{E}$. Seja $m_{E}$ o disco fechado em $\sum^{\varkappa}$ cujo bordo é $c_{E}$. Se $c_{E} \cap j_{E} \neq \emptyset$, então os pontos dessa interseção correspondem a órbitas homoclínicas a $o_{E}$. Ver figura 6-6.

Mas se $c_{E} \cap j_{E}=\emptyset$ então podemos considerar as iteradas $\psi^{i}\left(m_{E}\right)$. Como $\psi$ preserva área 


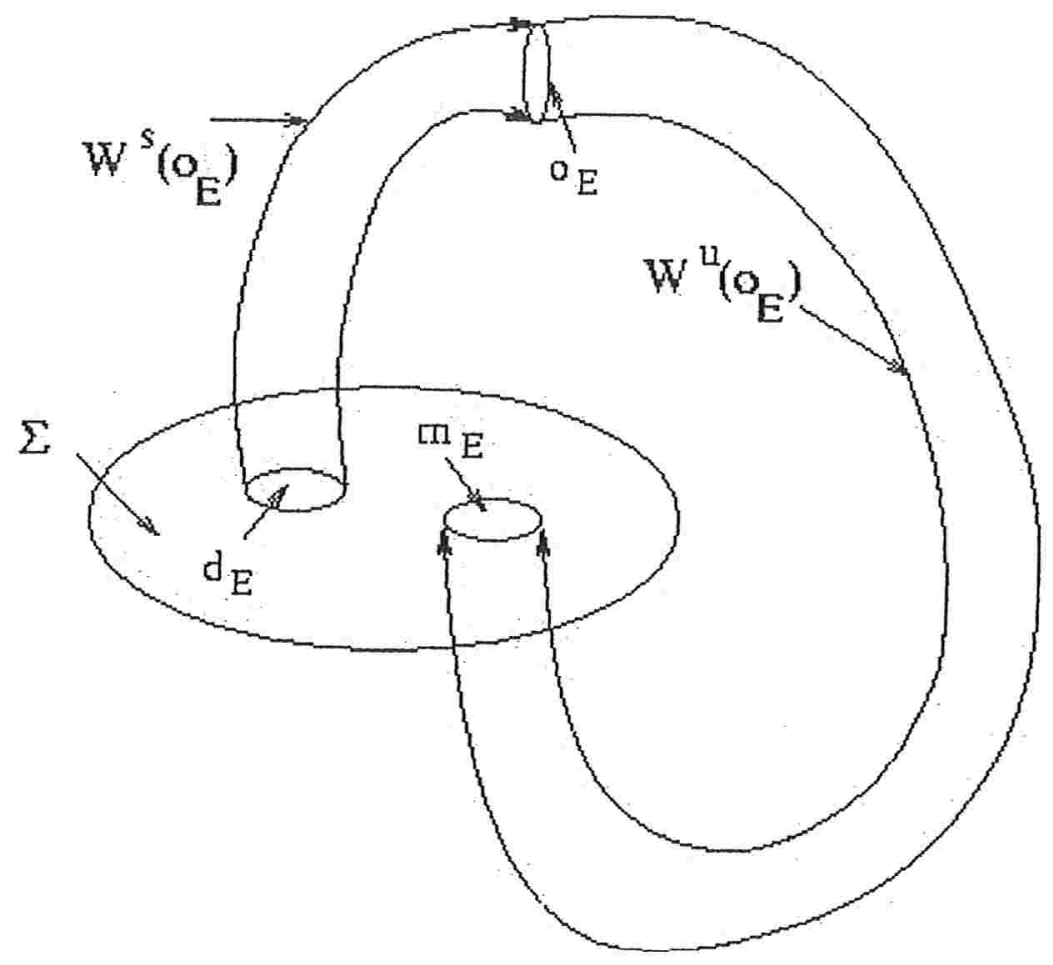

Figura 6-6: As intersecções de $\psi^{n}\left(j_{E}\right)$ com $c_{E}$ corresponde a órbitas homoclínicas a $O_{E}$. 
e as áreas de $m_{E}$ e $\sum^{n}$ são finitas e diferentes de zero, então conforme feito na Proposição 3.I, existe $N \geq 0$ tal que $\psi^{N}\left(j_{E}\right) \cap c_{E} \neq \emptyset$. Tais pontos correspondem à órbitas homoclínicas a $o_{E}$ de $N$-pulsos. Se as varieades estável e instável de $o_{E}$ não coincidem, ou seja, se $c_{E} \neq \psi^{k}\left(j_{E}\right)$ para nenhum $k \geqq 0$, então, também conforme a proposição 3.1 , existem infinitos pontos em $\sum^{n} \backslash d_{E}$ tais que $\psi^{N}$ está bem definida e que correspondem a órbitas homoclínicas a $o_{E}$ de $2 N$-pulsos. Nesse caso, pode-se mostrar a existência de conjuntos cuja dinâmica é conjugada ao shift de Bernoulli, apresentando entropia topológica positiva. Veja mais detalhes em[BGS]. 


\section{Capítulo 7}

\section{Exemplos e Aplicações}

\subsection{Formas Quadráticas Positivas-definidas}

Quando uma hipersuperfície $S$ é definida como pré-imagem de um valor regular de uma função $H: \mathbb{R}^{4} \rightarrow \mathbb{R}$, vimos que a convexidade estrita de $S$ depende do Hessiano de $H$. Mais precisamente, vimos que uma condição necessária para que uma hipersuperfície seja estritamente convexa é que $H_{x x}$, o Hessiano de $H$, seja definido em $T_{x} S$ para todo $x \in S$.

Sem perda de generalidade podemos impor que o Hessiano de $H$ seja positivo-definido em $T_{x} S$, ou seja, para todo $v \in T_{x} S$ não nulo, $\left\langle H_{x x}(x) v, v\right\rangle>0$. Em geral o Hessiano não é definido em $T_{x} \mathbb{R}^{4}$, bastando, para a convexidade, ser definido apenas no subespaço $T_{x} S$.

Seja $L: \mathbb{R}^{4} \rightarrow \mathbb{R}^{4}$ um operador linear auto-adjunto. Seja $Q: \mathbb{R}^{4} \times \mathbb{R}^{4} \rightarrow \mathbb{R}$ a forma quadrática definida por $Q(v)=\langle L v, v\rangle$ onde $\langle$,$\rangle denota o produto interno usual de \mathbb{R}^{4}$. Seja $E$ um subespaço de $\mathbb{R}^{4}$ de dimensão 3 e $\left\{X_{i}, i=1,2,3\right\}$ uma base de $E$. Seja $W$ a matriz $3 \times 3$ simétrica, definida por

$$
W=\left(\left\langle L X_{i}, X_{j}\right\rangle\right)_{1 \leqq i, j \leqq 3}
$$

Então temos o seguinte Lema

Lema 7.1 A forma quadrática $Q$ é positiva-definida em $E$ se e somente se todos os autovalores de $W$ sâo positivos.

Prova. Ver [Mad]. 
Lema 7.2 Seja $W=\left(w_{i j}\right)_{1 \leqq i, j \leqq 3}$ uma matriz $3 \times 3$ simétrica. Então todos os autovalores de $W$ são positivos se e somente se as seguintes condições são satisfeitas

$\begin{aligned} \operatorname{det} W & >0 \\ \operatorname{tr} W & >0 \\ D_{W} & >0\end{aligned}$

onde $D_{W}=w_{11} w_{22}-w_{12}^{2}+w_{11} w_{33}-w_{13}^{2}+w_{22} w_{33}-w_{23}^{2}$.

Prova. É imediato a partir das relações de Girard para polinômios de grau 3.

\subsection{Hamiltonianos da forma $\frac{p_{x}^{2}+p_{y}^{2}}{2}+V(x, y)$}

Seja $H$ uma função Hamiltoniana da forma $H\left(x, y, p_{x}, p_{y}\right)=\frac{p_{x}^{2}+p_{y}^{2}}{2}+V(x, y)$, onde $V: \mathbb{R}^{2} \rightarrow \mathbb{R}$ é uma função $C^{k \geqq 2}$, conhecida como função potencial. Seja $z=\left(x, y, p_{x}, p_{y}\right)$ os coordenadas de $\mathbb{R}^{4}$ e seja $\pi: \mathbb{R}^{4} \rightarrow \mathbb{R}^{2}$ a projeção no plano $(x, y)$. Suponhamos que $S \subset H^{-1}(E)$ seja uma hipersuperfície compacta, homeomorfa a $S^{3}$ e regular em todo ponto exceto em $p_{c}$ que corresponde a um ponto de equilíbrio do campo $X_{H}$, ou seja, $\pi\left(p_{c}\right)$ é um ponto crítico de $V(x, y)$. O conjunto $B \stackrel{\text { def }}{=} \pi(S)$ é, portanto, homeomorfo a $D^{2}$, cujo bordo $\partial B=\{\pi(z), z \in S, z=$ $(x, y, 0,0)\}$ é uma curva regular exceto em $\pi\left(p_{c}\right)$. O interior de $B$ é dado por $\stackrel{\text { o def }}{=}\{\pi(z), z \in$ $\left.S, p_{x}^{2}+p_{y}^{2} \neq 0\right\}$ e se $(x, y) \in \stackrel{\circ}{B}$ então $V(x, y)<E$. Então temos

$$
\begin{aligned}
H_{x}(z)= & \left(V_{x}, V_{y}, p_{x}, p_{y}\right) \\
H_{x x}(z)= & \left(\begin{array}{llll}
V_{x x} & V_{x y} & 0 & 0 \\
V_{x y} & V_{y y} & 0 & 0 \\
0 & 0 & 1 & 0 \\
0 & 0 & 0 & 1
\end{array}\right)
\end{aligned}
$$

Seja $z \in S$ tal que $p_{x}^{2}+p_{y}^{2} \neq 0$, ou seja, $\pi(z) \in \stackrel{\circ}{B}$. Então uma base para $T_{z} S=\left[H_{x}(z)\right]^{\perp}$ é dada por

$$
X_{1}=\left(0,0,-p_{y}, p_{s}\right)
$$




$$
\begin{aligned}
& X_{2}=\left(p_{x}, p_{y},-V_{x},-V_{y}\right) \\
& X_{3}=\left(p_{y},-p_{x}, V_{y},-V_{x}\right)
\end{aligned}
$$

pois $X_{i}, i=1,2,3$ são linearmente independentes e ortogonais a $H_{x}$. Queremos verificar a convexidade local de $S$ em $z$. Observe que $H_{x x}$ tem dois autovalores iguais a 1 , logo o Hessiano de $H$ é definido em $T_{z} S$ se e somente se ele for positivo-definido em $T_{z} S$. A matriz $W$ definida em (7.1) é dada por

$$
\mathrm{W}=\left(\begin{array}{lll}
p_{1}^{2}+p_{2}^{2} & p_{2} V_{x}-p_{1} V_{y} & -p_{2} V_{y}-p_{1} V_{x} \\
p_{2} V_{x}-p_{1} V_{y} & V_{x x} p_{1}^{2}+2 p_{1} p_{2} V_{x y}+V_{y y} p_{2}^{2}+V_{x}^{2}+V_{y}^{2} & p_{1} p_{2}\left(V_{x x}-V_{y y}\right)+\left(p_{2}^{2}-p_{1}^{2}\right) V_{x y}^{2} \\
-p 2 V_{y}-p_{1} V_{x} & p_{1} p_{2}\left(V_{x x}-V_{y y}\right)+\left(p_{2}^{2}-p_{1}^{2}\right) V_{x y}^{2} & V_{x x} p_{2}^{2}+2 p_{1} p_{2} V_{x y}+V_{y y} p_{1}^{2}+V_{x}^{2}+V_{y}^{2}
\end{array}\right)
$$

onde $L=H_{x x}$. Então temos

$$
\begin{aligned}
\operatorname{det} W & =4(E-V)^{2}\left(2(E-V)\left(V_{x x} V_{y y}-V_{x y}^{2}\right)+V_{x x} V_{y}^{2}+V_{y y} V_{x}^{2}-2 V_{x} V_{y} V_{x y}\right) \\
\operatorname{tr} W & =2(E-V)\left(1+V_{x x}+V_{y y}\right)+2\left(V_{x}^{2}+V_{y}^{2}\right) \\
D_{W} & =4(E-V)^{2}\left(V_{x x}+V_{y y}+V_{x x} V_{y y}\right)+2(E-V)\left(1+V_{x x}+V_{y y}\right)\left(V_{x}^{2}+V_{y}^{2}\right)+\left(V_{x}^{2}+V_{y}^{2}\right)^{2}
\end{aligned}
$$

Para os pontos $z \in S, z \neq \pi\left(p_{c}\right)$ tais que $p_{x}=p_{y}=0$, ou seja, se $\pi(z) \in \partial B \backslash \pi\left(p_{c}\right)$, então tomamos como base de $T_{z} S$ os seguinte vetores

$$
\begin{aligned}
& X_{1}=(0,0,1,0) \\
& X_{2}=(0,0,0,1) \\
& X_{3}=\left(V_{y},-V_{x}, 0,0\right)
\end{aligned}
$$

e a matriz $W$ definida em (7.1) é dada por

$$
W=\left(\begin{array}{lll}
1 & 0 & 0 \\
0 & 1 & 0 \\
0 & 0 & V_{x x} V_{y}^{2}+V_{y y} V_{x}^{2}-2 V_{x} V_{y} V_{x y}
\end{array}\right)
$$


e, portanto,

$$
\begin{aligned}
\operatorname{det} W & =V_{x x} V_{y}^{2}+V_{y y} V_{x}^{2}-2 V_{x} V_{y} V_{x y} \\
\operatorname{tr} W & =2+V_{x x} V_{y}^{2}+V_{y y} V_{x}^{2}-2 V_{x} V_{y} V_{x y}=2+\operatorname{det} W \\
D_{W} & =1+2\left(V_{x x x} V_{y}^{2}+V_{y y} V_{x}^{2}-2 V_{x} V_{y} V_{x y}\right)=1+2 \operatorname{det} W
\end{aligned}
$$

Seja $S_{0}=S \backslash p_{c}$.

Proposição $7.1 W$ é positiva-definida em $T S_{0}$ se e somente se

$$
T_{W} \stackrel{\text { def }}{=} 2(E-V)\left(V_{x x} V_{y y}-V_{x y}^{2}\right)+V_{x x} V_{y}^{2}+V_{y y} V_{x}^{2}-2 V_{x} V_{y} V_{x y}>0
$$

em $B \backslash \pi\left(p_{c}\right)$.

Prova. Suponhamos que $T_{W}>0$ em todo ponto de $B \backslash \pi\left(p_{c}\right)$. Então, por (7.2) e (7.3), $\operatorname{det} W>0 \mathrm{em} B \backslash \pi\left(p_{c}\right)$. Isso é suficiente para que $W$ seja positiva-definida em $\{p \in S \mid \pi(p) \in$ $\left.\partial B \backslash \pi\left(p_{c}\right)\right\}$ pois $V=E$ em $\partial B$.

Se $p \in S$ e $\pi(p) \in \stackrel{\circ}{B}$ está suficientemente próximo de $\partial B \backslash \pi\left(p_{c}\right)$, então, por (7.2), temos que $\operatorname{tr} W>0$ e $D_{W}>0$ pois $V \stackrel{(x, y) \rightarrow \partial B}{\longrightarrow} E$ e $V_{x}^{2}+V_{y}^{2}>0$ em $\partial B \backslash \pi\left(p_{c}\right)$. Logo $W$ é positiva-definida em $p$. Como $\stackrel{\circ}{B}$ é conexo e $\operatorname{det} W>0 \mathrm{em} \stackrel{\circ}{B}$, nenhum autovalor de $W$ pode mudar de sinal em $\stackrel{\circ}{B}$ e, portanto, estes devem ser todos positivos em todo ponto de $\stackrel{\circ}{B}$. Logo $W$ é positiva-definida em todo $p \in S$ tal que $\pi(p) \in \stackrel{\circ}{B}$. Concluimos que $W$ em positiva-definida em $S \backslash p_{c}$. A recíproca é imediata.

Seja $R: B \rightarrow \mathbb{R}$ dada por $R(x, y)=\sqrt{(E-V(x, y))}$. Então $\left.R\right|_{\partial B}=0$ e $\left.R\right|_{B}>0$. A curva $\partial B$ é uma curva de Jordan $C^{k \geqq 2}$ em todo ponto diferente de $\pi\left(p_{c}\right)$.

Proposição $7.2 W$ é positiva-definida em $S \backslash p_{c}$ se e somente se $\partial B \backslash \pi\left(p_{c}\right)$ tem curvatura normal nấo nula e a função $R$ é $C^{2}$ localmente estritamente côncava em $\stackrel{\circ}{B}$.

Prova. A função $R$ é $C^{2}$ localmente estritamente côncava em $\stackrel{\circ}{B}$ se e somente se a matriz

$$
\tilde{W} \stackrel{\text { def }}{=}\left(\begin{array}{ll}
R_{x x} & R_{x y} \\
R_{x y y} & R_{y y}
\end{array}\right)
$$


é negativa-definida. Então temos

$$
\tilde{W}=-\frac{1}{4(E-V)^{\frac{3}{2}}}\left(\begin{array}{ll}
2 V_{x x}(E-V)+V_{x}^{2} & 2 V_{x y}(E-V)+V_{x} V_{y} \\
2 V_{x y}(E-V)+V_{x} V_{y} & 2 V_{y y}(E-V)+V_{y}^{2}
\end{array}\right)
$$

e, portanto, $\tilde{W}$ é negativa definida se e somente se $\operatorname{det} \tilde{W}>0$ e $\operatorname{tr} \tilde{W}<0$, ou seja

$$
\begin{aligned}
\operatorname{det} \tilde{W} & =\frac{2(E-V)\left(V_{x x} V_{y y}-V_{x y}^{2}\right)+V_{x x} V_{y}^{2}+V_{y y} V_{x}^{2}-2 V_{x} V_{y} V_{x y}}{16(E-V)^{3}}>0 \\
-\operatorname{tr} \tilde{W} & =\frac{2(E-V)\left(V_{x x}+V_{y y}\right)+V_{x}^{2}+V_{y}^{2}}{4(E-V)^{\frac{3}{2}}}>0
\end{aligned}
$$

Logo, $\tilde{W}$ é negativa-definida se e somente se $\left.\operatorname{det} W>0 \mathrm{em} \pi^{-1} \stackrel{\circ}{B}\right) \cap S$ já que $-\operatorname{tr} \tilde{W}>0$ para pontos próximos de $\partial B \backslash \pi\left(p_{c}\right)$.

Os pontos da curva regular $\partial B \backslash \pi\left(p_{c}\right)$ satisfazem a equação $V(x, y)=E$ e a curvatura é dada por

$$
k(x, y)=\frac{\left|V_{x x} V_{y}^{2}-2 V_{x y} V_{x} V_{y}+V_{y y} V_{x}^{2}\right|}{\left(V_{x}^{2}+V_{y}^{2}\right)^{\frac{3}{2}}}
$$

que, por (7.3) e continuidade da função $\operatorname{det} W$, é positiva se e somente se $\operatorname{det} W>0$ em $\pi^{-1}\left(\partial B \backslash \pi\left(p_{c}\right)\right) \cap S$.

A proposição 7.1 completa a demonstração.

\subsection{O Hamiltoniano Henon-Heilles}

Estudamos a função Hamiltoniana $H: \mathbb{R}^{4} \rightarrow \mathbb{R}$ dada por

$$
H\left(x, y, p_{x}, p_{y}\right)=\frac{p_{x}^{2}+p_{y}^{2}}{2}+V(x, y)
$$

onde $V(x, y)=\frac{x^{2}+y^{2}}{2}+b x^{2} y-\frac{y^{3}}{3}$ e $b$ é um parâmetro positivo. Quando $b=1$, temos o Hamiltoniano Henon-Heilles.

Independentemente do parâmetro $b$, o ponto $(0,1)$ é uma sela da função potencial $V$ e, para $p_{x}=p_{y}=0$, corresponde a um equilíbrio do tipo sela-centro para o fluxo Hamiltoniano associado à função $H$. O sela-centro $p_{c}=(0,1,0,0)$ está contido no nível de energia $M_{b} \stackrel{\text { def }}{=}$ 


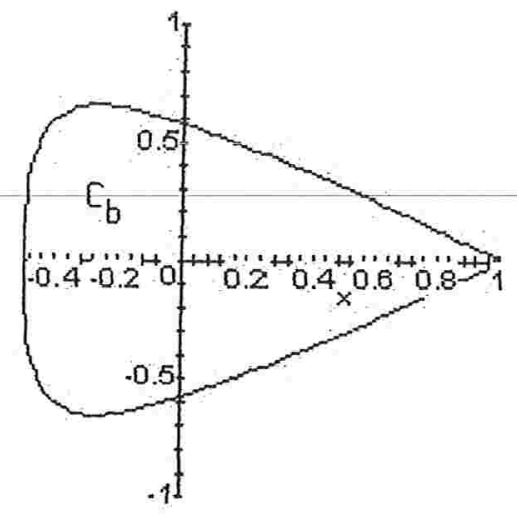

Figura ${ }^{\sim}$-1: $O$ bordo de $C_{b}$ é uma curva fechada simples, regular e com curvatura positiva em todo ponto excepto $\pi\left(p_{c}\right)$.

$\left\{H=\frac{1}{6}\right\}$. O nível de energia $M_{b}$ depende do parâmetro $b$. Seja $\pi: \mathbb{R}^{4} \rightarrow \mathbb{R}^{2}$ dada por $\pi\left(x, y, p_{x}, p_{y}\right)=(x, y)$. Se $0<b<1$, então $\pi(M)$ possui uma componente $C_{b}$ que é homeomorfa a $D^{2}$ e contém $\pi\left(p_{c}\right)$. O bordo de $C_{b}$ é uma curva fechada, não diferenciável apenas em $\pi\left(p_{c}\right)$. Para todo $(x, y) \in C_{b}$, temos $-\frac{1}{2} \leqq y \leqq 1$ e $x^{2}<\frac{1-3 y^{2}+2 y^{3}}{3+6 b y}$. Os pontos $(x, y) \in \partial C_{b}$ satisfazem $V(x, y)=\frac{1}{6}$. Ver figura $7-1$.

Seja $S_{b} \stackrel{\text { def }}{=} \pi^{-1}(C) \cap M_{b}$.

Lema $7.3 S e(y, b) \in D \stackrel{\text { def }}{=}\left(-\frac{1}{2}, 0\right) \times(0,1) e 8 b^{2} y^{3}+12 b y^{2}+6 y+4 b^{2}-3>0$ então $4 b y^{2}+$ $3 y+b y+b>0$.

Prova. Vamos mostrar que $8 b y^{2}+6 y+2 b y+2 b>0$ em $D$. Temos

$$
8 b y^{2}+6 y>3-4 b^{2}-4 b y^{2}-8 b^{2} y^{3}
$$

Logo

$$
8 b y^{2}+6 y+2 b y+2 b>-4 b y^{2}-8 b^{2} y^{3}+3-4 b^{2}+2 b y+2 b
$$

Basta agora mostrar que

$$
R(y, b) \stackrel{\text { def }}{=}-4 b y^{2}-8 b^{2} y^{3}+3-4 b^{2}+2 b y+2 b>0
$$


em $D$. Em $\partial D$, temos

$$
\begin{aligned}
R\left(-\frac{1}{2}, b\right) & =-3 b^{2}+3>0(\text { exceto em } b=1) \\
R(0, b) & =3-4 b^{2}+2 b=(1-b)(4 b+3)+b>0 \\
R(y, 0) & =3>0 \\
R(y, 1) & =-8 y^{3}-4 y^{2}+2 y+1
\end{aligned}
$$

Além disso, $\frac{\partial R(y, 1)}{\partial y}=-24 y^{2}-8 y+2$ e é simples ver que $\frac{\partial R(y, 1)}{\partial y}>0$ para $-\frac{1}{2}<y \leqq 0$. Logo $R(y, 1)>0$ para todo $-\frac{1}{2}<y \leqq 0$. Concluímos que $R(y, b)$ é uma função positiva em $\partial D$ exceto no ponto $\left(-\frac{1}{2}, 1\right)$ onde ela se anula. Para mostrarmos que $R>0$ em $D$ basta mostrarmos que $R(y, b)$ não possui ponto crítico em $D$, ou seja, as soluções de

$$
\left(-8 b y-24 b^{2} y^{2}+2 b,-4 y^{2}-8 b-18 b y^{3}+2 y+2\right)=(0,0)
$$

não estão contidas em $D$. Mas se $-8 b y-24 b^{2} y^{2}+2 b=0, b \neq 0$, então

$$
y_{ \pm}=\frac{-1 \pm \sqrt{1+3 b}}{6 b}
$$

Se $0<b<1$, então $y_{+}>0$ e $y_{-}<-\frac{1}{2}$ pois

$$
\begin{aligned}
9 b(b-1) & <0 \Leftrightarrow 9 b^{2}-6 b+1<3 b+1 \Leftrightarrow(3 b-1)^{2}<3 b+1 \\
& \Rightarrow 3 b-1<\sqrt{1+3 b} \Rightarrow \frac{-1-\sqrt{1+3 b}}{6 b}<-\frac{1}{2}
\end{aligned}
$$

Não existem, portanto, pontos críticos de $R(y, b)$ em $D$ concluindo que $R(y, b)>0$ em $D$.

Proposição 7.3 Para todo $0<b<1, S_{b}$ é uma hipersuperficie estritamente convexa em $\mathbb{R}^{4}$, homeomorfa a $S^{3}$, não-regular apenas em $p_{c}$.

Prova. É suficiente mostrar que

$$
2\left(V_{x x} V_{y y}-V_{x y}^{2}\right)\left(\frac{1}{6}-V\right)+V_{x}^{2} V_{y y}+V_{y}^{2} V_{x x}-2 V_{x} V_{y} V_{x y}>0
$$

para todo $(x, y) \in \stackrel{\circ}{C}_{b}$, o interior de $C_{b}$. Isso é equivalente a mostrar que 


$$
N(y, b)+F(y, b) x^{2}+G(y, b) x^{4}>0
$$

onde $N(y, b) \stackrel{\text { def }}{=}(1+y)(1-y)^{3}(1+2 b y), F(y, b) \stackrel{\text { def }}{=} 2 b(y-1)\left(2 b y^{2}+3 y+2 b y+2 b\right) \mathrm{e}$ $G(y, b) \stackrel{\text { def }}{=} 3 b^{2}(1+2 b y)$. Seja $B=\left(-\frac{1}{2}, 1\right) \times(0,1)$.

Resolvendo a equação para $x^{2}$

$$
N(y, b)+F(y, b) x^{2}+G(y, b) x^{4}=0
$$

obtemos

$$
x_{ \pm}^{2}=\frac{-K_{1}(y, b) \pm \sqrt{K_{2}(y, b)}}{b(3+6 b y)}
$$

onde

$$
\begin{aligned}
& K_{1}(y, b)=3 y^{2}+2 b y^{3}-3 y-2 b \\
& K_{2}(y, b)=(1+2 y)(1-y)^{2}\left(8 b^{2} y^{3}+12 b y^{2}+6 y+4 b^{2}-3\right)
\end{aligned}
$$

Como $N(y, b)>0$ e $G(y, b)>0$ em $B, x_{-}^{2}$ e $x_{+}^{2}$ têm o mesmo sinal. Estamos interessados, obviamente, apenas no caso $x_{ \pm}^{2}$ positivos e, portanto, assumimos que $K_{2}(y, b)>0$. Vamos agora mostrar que $x^{2}<x_{-}^{2}$ para todo $(x, y) \in \stackrel{\circ}{C}_{b}$. Para isso, é suficiente mostrar que $\frac{1-3 y^{2}+2 y^{3}}{3+6 b y}<x_{-}^{2}$ em $B$. Mas em $B$, temos

$$
\frac{1-3 y^{2}+2 y^{3}}{3+6 b y}<x_{-}^{2} \Leftrightarrow \sqrt{(1+2 y)\left(8 b^{2} y^{3}+12 b y^{2}+6 y+4 b^{2}-3\right)}<4 b y^{2}+3 y+b y+b
$$

e, portanto, pelo lema 7.3

$$
\frac{1-3 y^{2}+2 y^{3}}{3+6 b y}<x_{-}^{2} \Leftrightarrow 3 b y+y+b+1>0
$$

e é imediato verificar que $3 b y+y+b+1>0$ em $B$. 


\subsection{Um exemplo integrável}

O Hamiltoniano $I I: \mathbb{R}^{4} \rightarrow \mathbb{R}$ dado por

$$
H\left(x, y, p_{x}, p_{y}\right)=\frac{p_{x}^{2}+p_{y}^{2}}{2}+V(x, y)
$$

onde $V(x, y)=\frac{x^{2}+k y^{2}}{2}+\frac{1}{2}\left(x^{2}+y^{2}\right)^{2}$ é um exemplo de um sistema integrável cuja segunda integral primeira $F$ é dada por

$$
F\left(x, y, p_{x}, p_{y}\right)=-\frac{\left(x p_{y}-p_{x} y\right)^{2}}{2(k-1)}+p_{y}^{2}+k y^{2}+\frac{1}{2} y^{2}\left(x^{2}+y^{2}\right)
$$

Quando o parâmetro $k$ é negativo, a origem é uma sela da função $V(x, y)$ e, portanto, o ponto $p_{c} \stackrel{\text { def }}{=}(0,0,0,0)$ é um ponto de equilíbrio do tipo sela-centro do fluxo Hamiltoniano associado à função $H$. O ponto $p_{c}$ está contido no nível de energia $M_{k} \stackrel{\text { def }}{=}\{H=0\}$.

Seja $\pi: \mathbb{R}^{4} \rightarrow \mathbb{R}^{2}$ dada por $\pi\left(x, y, p_{x}, p_{y}\right)=(x, y)$. Então $\pi\left(M_{k}\right)$ é simétrico em relação aos eixos $x$ e $y$, e possui, para $y \geqq 0$, uma componente $C_{k}$ homeomorfa a $D^{2}$ cujo bordo $\partial C_{k}$ é regular em todos os pontos exceto na origem. Os pontos do bordo são dados por $\partial C_{k}=$ $\{(x, y) \mid y \geqq 0, V(x, y)=0\}$. Seja $S_{k} \stackrel{\text { def }}{=} \pi^{-1}\left(C_{k}\right)$.

Proposição 7.4 Para todo $k<0, S_{k}$ é uma hipersuperfície estritamente convexa em $\mathbb{R}^{4}$, homeomorfa a $S^{3}$, nấo-regular apenas em $p_{c}$.

Prova. Vamos mostrar que

$$
2\left(V_{x x} V_{y y}-V_{x y}^{2}\right)\left(\frac{1}{6}-V\right)+V_{x}^{2} V_{y y}+V_{y}^{2} V_{x x}-2 V_{x} V_{y} V_{x y}>0
$$

para todo $(x, y) \in C_{k} \backslash\{(0,0)\}$. Isso é equivalente a mostrar que

$$
K \stackrel{\text { def }}{=} 2 y^{6}+6 x^{2} y^{4}-3 y^{4}+6 k y^{4}-y^{2}+k y^{2}+6 k x^{2} y^{2}+6 x^{4} y^{2}+2 x^{6}+3 x^{4}<0
$$

Sabemos que em $C_{k}, V(x, y) \leqq 0$, ou seja,

$$
x^{2}+k y^{2}+x^{4}+2 x^{2} y^{2}+y^{4} \leqq 0
$$


Então, temos

$$
\begin{aligned}
& K_{1} \stackrel{\text { def }}{=} 2 x^{2} y^{2}+2 k y^{4}+2 x^{4} y^{2}+4 x^{2} y^{4}+2 y^{6} \leqq 0 \\
& K_{2} \stackrel{\text { def }}{=} 2 x^{4}+2 k x^{2} y^{2}+2 x^{6}+4 x^{4} y^{2}+2 x^{2} y^{4} \leqq 0
\end{aligned}
$$

Logo

$$
K-K_{1}-K_{2}=-12 x^{2} y^{4}-3 y^{4}+4 k y^{4}-y^{2}+k y^{2}+3 k x^{2} y^{2}+x^{2}\left(x^{2}+k y^{2}\right)
$$

Mas $x^{2}+k y^{2}<0$ em $C_{k} \backslash\{(0,0)\}$ pois resolvendo a equação $V(x, y) \leqq 0$, obtemos

$$
x^{4}+\left(2 y^{2}+1\right) x^{2}+k y^{2}+y^{4} \leqq 0
$$

e, portanto, $0 \leqq x^{2} \leqq \frac{-\left(2 y^{2}+1\right)+\sqrt{4 y^{2}(1-k)+1}}{2} . \operatorname{Mas} \sqrt{4 y^{2}(1-k)+1}<1+2 y^{2}-2 k y^{2}$ e, então $x^{2}<-k y^{2}$ de onde concluímos que $K-K_{1}-K_{2}<0$. Com isso, temos que $K<K_{1}+K_{2} \leqq 0$ em $C_{k} \backslash\{(0,0)\}$.

O hiperplano $H_{0} \stackrel{\text { def }}{=}\{y=0\}$ é tal que $H_{0} \cap S_{k}=p_{c}$. 


\section{Capítulo 8}

\section{Hipersuperfícies de Contato em $\mathbb{R}^{4}$}

Consideramos $\left(x_{1}, x_{2}, x_{3}, x_{4}\right)$ as coordenadas em $\mathbb{R}^{4}$. Sejam $x_{i}: \mathbb{R}^{4} \rightarrow \mathbb{R}, i=1,2,3,4$, as projeções sobre as coordenadas de $\mathbb{R}^{4}$. Para cada $x \in \mathbb{R}^{4}$, definimos uma base $\left\{\frac{\partial}{\partial x_{i}}\right\}_{i=1,2,3,4}$ para $T_{x} \mathbb{R}^{4} \simeq \mathbb{R}^{4}$ dada por $d x_{i}\left(\frac{\partial}{\partial x_{j}}\right)=\delta_{i j}$. É possível trivializar $T \mathbb{R}^{4} \simeq \mathbb{R}^{4} \times \mathbb{R}^{4}$ usando essa base em todo $x \in \mathbb{R}^{4}$. Sejam $\left(v_{1}, v_{2}, v_{3}, v_{4}\right)$ as coordenadas em $T_{x} \mathbb{R}^{4}$ após feita essa trivialização.

Dados $x \in \mathbb{R}^{4}$ e $v \in T_{x} \mathbb{R}^{4} \simeq \mathbb{R}^{4}$, definimos

$$
\lambda_{0}(x)(v) \stackrel{\text { def }}{=} \frac{1}{2} \sum_{j=1}^{2}\left[x_{j}(x) d x_{j+2}(v)-x_{j+2}(x) d x_{j}(v)\right]
$$

a 1-forma definida em $\mathbb{R}^{d}$ onde $d x_{j}(v)=v_{j}$. Então

$$
\omega_{0} \stackrel{\text { def }}{=} d \lambda_{0}=\sum_{j=1}^{2} d x_{j} \wedge d x_{j+2}
$$

é uma 2-forma exata e não-degenerada em $\mathbb{R}^{4}$ conhecida como forma simplética canônica de $\mathbb{R}^{4}$. O par $\left(\mathbb{R}^{4}, \omega_{0}\right)$ é, portanto, uma variedade simplética.

Seja $S \subset \mathbb{R}^{4}$ uma hipersuperfície regular. Definimos em $S$ a 3 -forma

$$
\nu_{0} \stackrel{\text { def }}{=} \lambda_{0}^{S} \wedge d \lambda_{0}^{S}
$$

onde $\lambda_{0}^{S}$ é a restrição de $\lambda_{0}$ a $T S$.

Suponha que $\nu_{0}$ seja não-degenerada, ou seja, $\nu_{0}$ define uma forma de volume em $S$. Então 


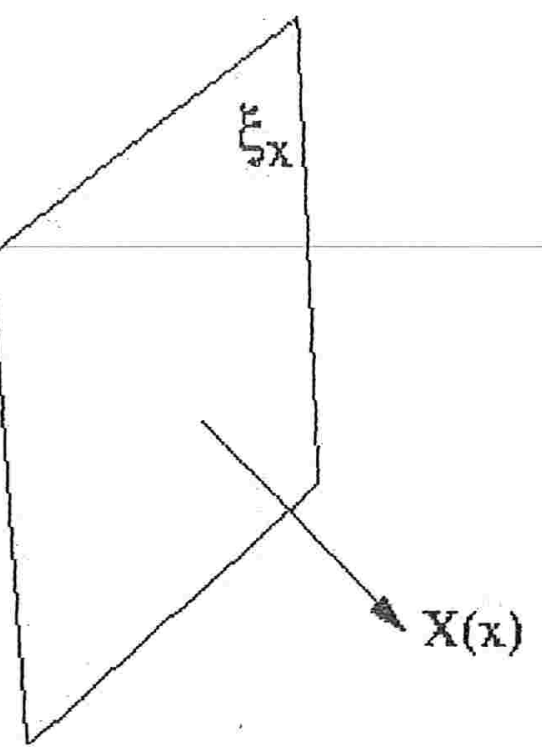

Figura 8-1: Numa hipersuperfície convexa que contém a origem, o campo Hamiltoniano é transversal á estrutura de contato

chamamos $S$ de hipersuperfíce de contato em $\left(\mathbb{R}^{4}, \omega_{0}\right)$. A 1-forma $\lambda_{0}$ é chamada, neste caso, de forma de contato. Associada à forma de contato $\lambda_{0}$, definimos a seguinte distribuição de hiperplanos $\xi$ em $T S$ : para cada $x \in S$ definimos o hiperplano $\xi_{x} \subset T_{x} S$ dado por

$$
\xi_{x}=\operatorname{ker} \lambda_{0}^{S}(x)
$$

O fibrado linear $\xi$ é chamado de estrutura de contato.

Lema 8.1 Para todo $x \in S, \xi_{x}$ é transversal a $X_{3}(x)$ (definido em (4.2)) em $T_{x} S$, onde $\xi_{x}$ é a fibra de $\xi$ em $x$.

Prova. Sejam $v, w \in T_{x} S$ tais que $\left\{x, w, X_{3}(x)\right\}$ seja linearmente independente. Sabemos que $d \lambda_{0}^{S}\left(X_{3}(x), v\right)=\left\langle J_{0} X_{3}(x), v\right\rangle=\langle N(x), v\rangle=0 \forall v \in T_{x}(S)$ onde $N(x)$ é um vetor unitário normal a $S$ em $x$. A não-degenerescência de $\nu_{0}$ (ver definição em (8.2)) implica que $\lambda_{0}^{S}(x)\left(X_{3}(x)\right) d \lambda_{0}^{S}(x)(v, w) \neq 0$ donde $\lambda_{0}^{S}(x)\left(X_{3}(x)\right) \neq 0$. Logo $X_{3}(x) \notin \xi_{x}$ e, portanto, $X_{3}(x)$ é transversal a $\xi_{x}$ em $T_{x} S$. Ver figura 8=1. 
Proposição 8.1 Seja $S \subset \mathbb{R}^{4}$ uma hipersuperfície difeomorfa $a S^{3}$, estritamente convexa $e$ com a origem em seu interior. Então $S$ é uma hipersuperfície de contato em $\left(\mathbb{R}^{4}, \omega_{0}\right)$.

Prova. Devemos mostrar que $\nu_{0}$ definida em (8.2) é não-degenerada, ou seja, $\nu_{0}(x)(v, w, z) \neq$ 0 para todo $x \in S$ e $v, w, z \in T_{x} S, v, w$ e $z$ linearmente independentes. Temos

$$
\begin{aligned}
& \nu_{0}(x)(v, w, z)=\lambda_{0}^{S}(x)(v) d \lambda_{0}^{S}(x)(w, z)-\lambda_{0}^{S}(x)(w) d \lambda_{0}^{S}(x)(v, z)+\lambda_{0}^{S}(x)(z) d \lambda_{0}^{S}(x)(v, w) \\
& \nu_{0}(x)(v, w, z)=\left|\begin{array}{llll}
x_{1} & x_{2} & x_{3} & x_{4} \\
v_{1} & v_{2} & v_{3} & v_{4} \\
w_{1} & w_{2} & w_{3} & w_{4} \\
z_{1} & z_{2} & z_{3} & z_{4}
\end{array}\right|
\end{aligned}
$$

A condição de não-degenerescência de $\nu_{0}$ é equivalente, portanto, à transversalidade da reta $O x$ com o hiperplano $H_{x}$ tangente a $S$ em $x$. Se $O x$ não for transversal a $H_{x}$ então $O x \subset H_{x}$ o que implica que $H_{x}$ contém a origem, mas a origem está contida em $\stackrel{\circ}{B}_{S}$, o interior de $S$, uma contradição, pois $H_{x}$ é um plano suporte de $B_{S}$.

Consideremos a partir de agora hipersuperfícies $S$ de $\mathbb{R}^{4}$ difeomorfas a $S^{3}$, estritamente convexas e com a origem em seu interior.

Podemos associar à variedade de contato $(S, \lambda)$ um campo de vetores $X$ em $S$ dado por

$$
\begin{aligned}
d \lambda(X, \cdot) & =0 \\
\lambda(X) & =1
\end{aligned}
$$

Esse campo é conhecido como campo de vetores de Reeb e é fácil mostrar que é equivalente ao campo Hamiltoniano em $S$. O campo de Reeb é transversal à estrutura de contato $\xi=\{\lambda=0\}$ e portanto $T_{x} S=\xi_{x} \oplus \mathbb{R} X(x)$ para todo $x \in S$. Além disso, é possível encontrar uma função Hamiltoniana $H: \mathbb{R}^{4} \backslash\{0\} \rightarrow \mathbb{R}$ tal que o campo Hamiltoniano associado a $H$ é igual ao campo de Reeb em $S$. A função $H$ é construída da seguinte forma: se $x \in \mathbb{R}^{4}, x \neq 0$, consideremos a semi-reta $r_{x}$ que começa na origem e passa por $x$. A reta $r_{x}$ intersecta $S$ em exatamente 1 ponto $p_{x} \in S$. Então existe um único $d_{x}>0$ tal que $x=d_{x} p_{x}$. Podemos então definir $H: \mathbb{R}^{4} \backslash\{0\} \rightarrow \mathbb{R}^{+}$por $H(x)=\frac{1}{d_{x}^{2}}$. Não é difícil mostrar que $H$ é uma função homogênea de grau $-2, H_{\left.\right|_{S}}=1$ e o campo Hamiltoniano associado a $H$ coincide com o campo de Reeb em 
$\left(S, \lambda_{0}\right)$. Ver [HZW].

Uma variedade de contato muito importante é $\left(S^{3}, \lambda_{0}\right)$. O campo de Reeb neste caso é muito simples: todas as órbitas são periódicas e degeneradas, constituindo a chamada fibração de Hopf. Se $S$ é uma hipersuperfície estritamente convexa em $\mathbb{R}^{4}$ com a origem no seu interior, podemos associar a $S^{3}$ uma nova forma de contato $\lambda=f \lambda_{0}$ de modo que o campo de Reeb em $\left(S^{3}, \lambda\right)$ seja equivalente ao campo de Reeb em $\left(S, \lambda_{0}\right)$. A função $f: S^{3} \rightarrow \mathbb{R}$ satisfaz $f(x)=d_{x}^{2}>0$. Uma prova sobre essa equivalência é dada em [HZW].

O estudo sobre a existência de órbitas periódicas e seçôes globais em hipersuperfícies estritamente convexas é geralmente feito em $S^{3}$ com a forma de contato $\lambda=f \lambda_{0}$ cujo campo de Reeb é equivalente ao campo Hamiltoniano em $S$. A estrutura de contato $\xi$ de $\left(S^{3}, \lambda\right)$ é a mesma que a de $\left(S^{3}, \lambda_{0}\right)$.

\subsection{Curvas Pseudo-Holomorfas}

Seja $\left(S^{3}, \lambda\right)$ uma variedade de contato tal que $\lambda=f \lambda_{0}, f: S^{3} \rightarrow \mathbb{R}, f>0$. Seja $\pi: T S^{3} \rightarrow \xi$ a projeção de $T S^{3}=\xi \oplus \mathbb{R} X_{\lambda}$ em $\xi$ ao longo de $X_{\lambda}$, onde $X_{\lambda}$ é o campo de Reeb em $\left(S^{3}, \lambda\right)$. É possível definir em $\xi$ uma operação $J: \xi \rightarrow \xi, J^{2}=-I d$, tal que em cada ponto $x \in S^{3}$ temos que a forma bilinear

$$
g_{J}(x)(h, k) \stackrel{\text { def }}{=} d \lambda(h, J(x) k)
$$

é positiva definida em $\xi_{x}$. Denotamos $|h|_{J}^{2}=g_{J}(x)(h, h)$. Chamamos $J$ com esta propriedade de estrutura complexa compatível em $\xi$.

Associada a $J$, definimos a estrutura quase complexa $\tilde{J}$ em $S^{3} \times \mathbb{R}$ dada por

$$
\widetilde{J}(x, a)(h, k)=\left(-\lambda(x) h, J(x) \pi h+k X_{\lambda}(x)\right)
$$

para todo $(h, k) \in T_{(x, a)} S^{3} \times \mathbb{R}$. É fácil ver que $\tilde{J}^{2}=-I d$.

Chamaremos de curvas pseudo-holomorfas a aplicações $\tilde{u}=(u, a): \mathbb{C} \rightarrow S^{3} \times \mathbb{R}$ que satisfazem

$$
\tilde{J} \circ D \tilde{u}=D \tilde{u} \circ i
$$


Em coordenadas $z=s+i t \in \mathbb{C}$, essa equarsão é equivalente a

$$
\begin{aligned}
a_{s} & =\lambda\left(u_{t}\right) \\
a_{t} & =-\lambda\left(u_{s}\right) \\
\pi u_{s}+J(u) \pi u_{t} & =0
\end{aligned}
$$

Definimos a energia $E(\widetilde{u})$ de uma solução por

$$
E(\tilde{u})=\sup _{\varphi \in \Sigma} \int_{\mathbb{C}} \tilde{u}^{*} d \lambda_{\varphi}
$$

onde $\sum=\left\{\varphi: \mathbb{R} \rightarrow[0,1], \varphi^{\prime}(x) \geqq 0\right\}$ e $\lambda_{\varphi}(x, a)(h, k) \stackrel{\text { def }}{=} \varphi(a) \lambda(x) h$.

Em coordenadas temos

$$
d \lambda_{\varphi}=\left[\varphi^{\prime}(a)\left(a_{s}^{2}+a_{t}^{2}\right)+\varphi(a)\left|\pi u_{s}\right|_{J}^{2}\right] d s \wedge d t
$$

As soluções não constantes que satisfazem $E(\tilde{u})<\infty$ estão diretamente ligadas à existência de órbitas periódicas e seções globais do campo de Reeb, e portanto, também para o campo Hamiltoniano em $S$. Veja [HZW] e [HZW2] para mais detalhes.

Dizemos que a forma de contato $\lambda$ é dinamicamente convexa se o índice de Conley-Zehnder de qualquer órbita periódica de $X_{\lambda}$ é maior ou igual a 3 .

O principal resultado de [HZW2] é

Teorema 8.1 Se $\lambda=f \lambda_{0}$ é uma forma de contato dinamicamente convexa em $S^{3}$ então existe uma órbita periódica $P$ do campo de Reeb $X_{\lambda}$ cujo índice de Conley-Zehnder é 3, considerando seu período mínimo T. Além disso, dada uma estrutura complexa $J$ compatível em $\xi$, existe um difeomorfismo

$$
\Phi: S^{1} \times \mathbb{C} \rightarrow S^{3} \backslash P
$$

tal que a aplicação $u_{\tau}=\Phi(\tau, \cdot): \mathbb{C} \rightarrow S^{3} \backslash P$ é um mergulho transversal a $X_{\lambda}$ e tem a órbita periódica $P$ como seu limite assintótico

$$
\lim _{R \rightarrow \infty} u_{\tau}\left(R . e^{2 \pi i t}\right)=P_{0}\left(T_{0} t\right) \text { in } C^{\infty}\left(S^{1}\right)
$$


A aplicação $u_{\tau}$ é a projeçấo canônica em $S^{3}$ de uma curva pseudo-holomorfa de energia finita, a 2-forma $d \lambda_{\mid u_{\tau}(\mathbb{C})}$ é não degenerada e sua área satisfaz $\int_{\mathbb{C}} u_{\tau}^{*} d \lambda=T$. Para cada $\tau, u_{\tau}(\mathbb{C})$ é uma seção global para o fluxo de $X_{\lambda}$.

O difeomorfismo $\Phi$ é chamado de "decomposição do livro aberto" de $S^{3} \backslash P$.

A forma bilinear $T S^{3}$ dada por

$$
\langle h, k\rangle_{2}=\lambda(h) \lambda(k)+d \lambda(\pi h, \pi J(x) k)
$$

é positiva definida e, portanto, temos uma nova métrica em $S^{3}$, onde denotaremos a norma de um vetor associada a essa métrica por $\|v\|_{2}^{2}=\langle v, v\rangle_{2}$. Podemos falar em ângulo $\angle h, k \in[0, \pi]$ entre os vetores $h$ e $k$, usando a métrica $\langle\cdot, \cdot\rangle_{2}$, ou seja,

$$
\cos (\angle h, k)=\frac{\langle h, k\rangle_{2}}{\|h\|_{2}\|k\|_{2}}
$$

É fácil ver que $\angle X_{\lambda}, v=\frac{\pi}{2}$ para todo $v \in \xi$. Definimos o ângulo $\angle \zeta, v \in\left[0, \frac{\pi}{2}\right]$ entre um plano $\zeta \subset T S^{3}$ e um vetor $v \in T S^{3}$ como sendo o menor ângulo entre um vetor $w \in \zeta$ e o vetor $v$. Temos, portanto, $\angle \xi, X_{\lambda}=\frac{\pi}{2}$.

A função $a: \mathbb{C} \rightarrow \mathbb{R}$, correspondente à componente $\mathbb{R}$ de uma curva pseudo-holomorfa de energia finita é limitada inferiormente e $a\left(z_{k}\right) \rightarrow \infty$ se $\left|z_{k}\right| \rightarrow \infty$. Além disso apresenta várias relações com a componente $u: \mathbb{C} \rightarrow S^{3}$.

Consideramos em $\mathbb{C}$ a métrica Euclidiana. Em primeiro lugar, por (8.4), temos

$$
\Delta a=\left|\pi u_{s}\right|_{J}^{2}>0
$$

onde $\Delta$ é o operador de Laplace. Portanto, $a$ é uma função sub-harmônica. Seja $\nabla a$ o gradiente de $a$. É fácil ver que

$$
D u \nabla a \in \xi
$$

ou seja, a direção do gradiente de $a$ é a direção onde a forma $\lambda$ se anula em $S^{3}$. Por outro lado, na direção $i \nabla a=\left(-a_{t}, a_{s}\right)$ temos o menor ângulo possível entre um vetor no espaço tangente a $u$ e $X_{\lambda}$, ou seja, $\angle T u, X_{\lambda}=\angle D u i \nabla a, X_{\lambda}$. Mais precisamente, esse ângulo é dado 
por

$$
\cos \angle T u, X_{\lambda}=\frac{|\nabla a|^{2}}{\Delta a+|\nabla a|^{2}}
$$

\subsection{Um sistema de coordenadas importante e a hipótese}

Apresentaremos agora um sistema de coordenadas que trivializa a forma de contato $\left(S^{3}, \lambda\right)$ e definiremos uma estrutura complexa em ker $\lambda$ que simplificará as equações das curvas pseudoholomorfas. Temos como objetivo preparar algumas ferramentas para uma possível continuidade deste trabalho no sentido de generalizar o teorema 6.2.

Seja $w:[a, b] \rightarrow S^{3}$ uma solução do campo de Reeb $X_{\lambda}$ tal que $w$ é um mergulho. Podemos encontrar uma vizinhança $U$ de $w([a, b])$ em $S^{3}$, uma vizinhança $V$ de $\{0\} \times\left[0, E_{1}\right]$ em $\mathbb{R}^{2} \times\left[0, E_{1}\right]$ e um difeomorfismo $\varphi: V \rightarrow U$ tal que

$$
\lambda^{\prime}=\varphi^{*} \lambda=d z+x d y
$$

onde $(x, y) \in \mathbb{R}^{2}$ e $z \in[a, b]$. Temos, portanto, $\varphi^{*} d \lambda=d \varphi^{*} \lambda=d \lambda^{\prime}=d x \wedge d y$.

O campo de Reeb em $V$ é constante, dado por $(0,0,1)$ e $\varphi\left(\{0\} \times\left[0, E_{1}\right]\right)=w\left(\left[0, E_{1}\right]\right)$.

A estrutura de contato $\zeta$, nas novas coordenadas, dada pelo núcleo da 1-forma (8.6) é gerada em cada ponto pelos vetores $e_{1}=(1,0,0)$ e $e_{2}=(0,1,-x)$. A projeção $\pi^{\prime}: T_{p} V \rightarrow \zeta_{p}$ ao longo do campo de Reeb é dada por

$$
\pi^{\prime}\left(h_{x}, h_{y}, h_{z}\right)=\left(h_{x}, h_{y},-x h_{y}\right)=h_{x} e_{1}+h_{y} e_{2}
$$

Podemos definir uma estrutura complexa $J^{\prime}: \zeta \rightarrow \zeta$ por

$$
\begin{aligned}
& J^{\prime} e_{1}=e_{2} \\
& J^{\prime} e_{2}=-e_{1}
\end{aligned}
$$

Portanto temos

$$
J^{\prime} \pi^{\prime}\left(h_{x}, h_{y}, h_{z}\right) \doteq-h_{y} e_{1}+h_{x} e_{2}
$$




$$
d \lambda^{\prime}\left(J^{\prime} \pi^{\prime} h, \pi^{\prime} k\right)=h_{x} k_{x}+h_{y} k_{y}
$$

Seja $\tilde{u}=(u, a): \mathbb{C} \rightarrow S^{3} \times \mathbb{R}$ uma curva pseudo-holomorfa com energia finita $E>0$. Então, nas coordenadas em $V \times \mathbb{R}$, pelas equações (8.4), temos

$$
\begin{aligned}
& x_{s}=y_{t} \\
& x_{t}=-y_{s} \\
& a_{s}=z_{t}+x y_{t} \\
& a_{t}=-z_{s}-x y_{s}
\end{aligned}
$$

É imediato verificar que

$$
\begin{aligned}
a_{s s}+a_{t t} & =x_{s}^{2}+x_{t}^{2}>0 \\
z_{s s}+z_{t t} & =0
\end{aligned}
$$

O campo $v_{0} \stackrel{\text { def }}{=} D u i \nabla a$, que é push-forward do campo $i \nabla a$, definido em $\mathbb{C}$, é dado por

$$
v_{0}=-a_{t} u_{s}+a_{s} u_{t}
$$

As curvas integrais do campo $v_{0}$ preservam a componente $a$.

Devido à transversalidade de $u$ com o campo de Reeb, temos que $z=z(x, y)$, ou seja, cada componente conexa da projeção da curva pseudo-holomorfa em $V$ é gráfico de uma função $f$ definida num subconjunto do plano $x y$ sobre a componente $z$. Seja $D$ uma dessas componentes conexas e suponhamos que $D$ intersecta os conjuntos $Z_{0} \stackrel{\text { def }}{=}\{z=0\}$ e $Z_{1} \stackrel{\text { def }}{=}\left\{z=E_{1}\right\}$. Então, pela mudança de coordenadas $(s, t) \rightarrow(x, y)$, que satisfaz $u(s, t)=(x, y, z(x, y))$, temos que

$$
\begin{aligned}
& a_{x x}+a_{y y}=1 \\
& z_{x x}+z_{y y}=0
\end{aligned}
$$


A projeção do campo $v_{0}$ no plano $x y$ é equivalente a campo $\tilde{v}_{0}$, dado por

$$
\tilde{v}_{0}=\left(z_{x}, z_{y}+x\right)
$$

É imediato que o campo $\tilde{v}_{0}$ preserva ćrea.

Seja $\gamma$ uma curva integral do campo vo, contida num nível regular da função $a$. Então temos

$$
\int_{\gamma} \lambda^{\prime}=\Delta z+\int_{\gamma} x d y
$$

Se $\gamma$ satisfaz $\left|\int_{\gamma} x d y\right|<\varepsilon$ então

$$
\int_{\gamma} \lambda^{\prime}>\Delta z-\varepsilon
$$

de modo que se $E_{0}>E+\varepsilon$, então a curva $\gamma$ não pode intersectar ambos os conjuntos $Z_{0}$ e $Z_{1}$, pois caso contrário teríamos

$$
\int_{\gamma} \lambda^{\prime}>E
$$

Mas sabemos que isso não é possível pois, como a função $a: \mathbb{C} \rightarrow \mathbb{R}$ é coerciva, o conjunto de nível de uma valor regular de $a$ é uma união finita de curvas fechadas simples e, pelo Teorema de Stokes, temos que

$$
\int_{\gamma} \lambda^{\prime}=\int_{\widetilde{\gamma}} u^{*} \lambda<\int_{C} u^{*} \lambda=\int_{i n t(C)} u^{*} d \lambda<E
$$

onde $\tilde{\gamma}=u^{-1}(\gamma) \subset C$, que é uma curva fechada simples, contida em um nível da função $a$. Lembramos que $u^{*} \lambda(i \nabla a)>0$ e, por isso, vale a primeira desigualdade em (8.7).

A hipótese $(H 1)$ introduzida no teorema 6.2 está diretamente relacionada a este fato. Mais precisamente, a solução $w$ pode ser escolhida como um ramo da variedade estável do sela-centro e se não existe órbita homoclínica ao equilíbrio, então podemos escolher $E_{0}$ arbitrariamente grande. Como já vimos, a órbita periódica que é bordo da seção global do sistema regularizado é sempre a mesma e, portanto, tem ação fixa igual a $E$. Portanto, pela análise do campo $\widetilde{v}_{0}$, se provarmos que existe pelo menos uma solução que intersecta os conjuntos $\widetilde{Z}_{0}$ e $\widetilde{Z}_{1}$ (as projeções de $Z_{0} \cap D$ e $Z_{1} \cap D$ no plano $x y$, respectivamente) teríamos uma contradição, conforme visto 


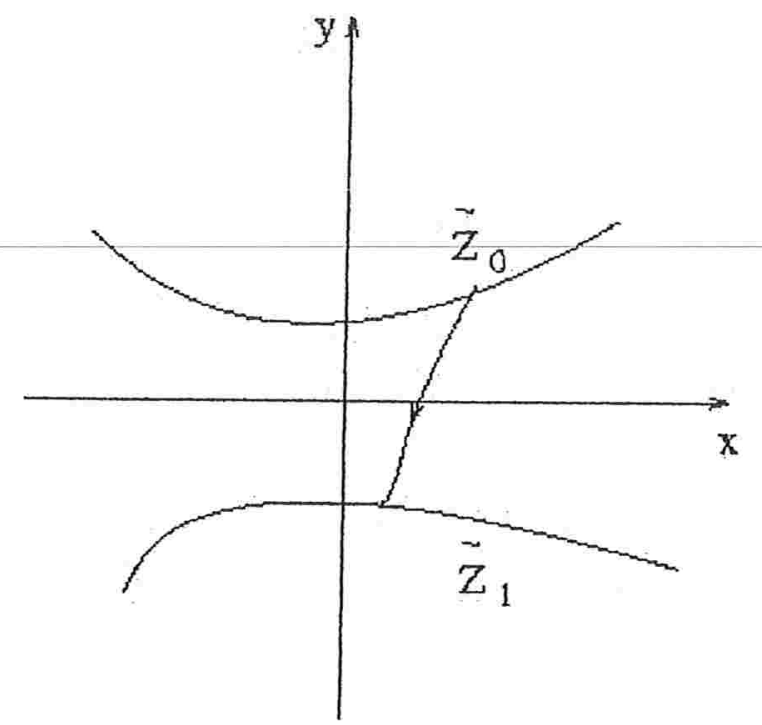

Figura $8-2$ :

acima. Ver figura 8-2.

Um campo de pesquisa importante, que daria uma continuidade natural a este trabalho, é, portanto, o estudo mais concentrado nas propriedades de curvas pseudo-holomorfas em $S^{3}$ com a finalidade de tornar a hipótese $(H 1)$ desnecessária. O teorema 6.2 seria, portanto, mais geral e suas hipóteses de convexidades ficariam relativamente fáceis de serem verificadas conforme feito nos exemplos de Hamiltonianos do tipo "energia cinética + potencial".

Da mesma forma, poderíamos eliminar a hipótese (H2) do teorema 6.3 e provarmos, apenas com hipóteses de convexidade do nível de energia do sela-centro, a existência de uma infinidade de órbitas periódicas e órbitas homoclínicas às órbitas periódicas da variedade central do selacentro. 


\section{Referências Bibliográficas}

[Ar] Arnold, V.I. (1978) Mathematical methods in classical mechanics. Springer-Verlag, Berlin.

[BGS] Bernard, P., Grotta-Ragazzo, C., Salomao, P.A.S., Homoclinic orbits near saddle-center fixed points of Hamiltonian systems with two degrees of freedom, será publicado na Asterisque em 2003. Arquivo disponfvel em www.geocities.com/pedrosalomao.

[Bus] Busemann, H., (1958) Convex surfaces, Interscience Tracts in Pure and App.Math., $\mathrm{n}^{\circ}$ 6 Interscience Publishers, Inc., New York; Interscience Publishers Ltd., London, 196 pp.

[CPR] Churchill, R. C., Pecelli, G., Rod, D.L., (1977) Hyperbolic structures in Hamiltonian systems, Rocky Mountain Journal of Mathematics, v.7, nº 3, pp 439-444.

[Con] Conley, C., (1968) Twist mappings, linking, analyticity, and periodic solutions which pass close to an unstable periodic solution, Topological Dynamics (Sympos., Colorado State Univ., Ft. Collins, Colo., 1967), pp. 129-153. Benjamin, New York.

[Con2] Conley, C., (1969) On the ultimate behavior of orbits with respect to an unstable critical point I. Oscillating, assymptotic and capture orbits, Journ. Diff. Eqns, 5, pp 136-158.

[CZ] Conley, C., Zehnder, E., (1984) Morse type index theory for flows and periodic solutions for Hamiltonian systems, Comm. Pure App. Math., 37, pp 207-253.

[F] Franks, J., (1996) Area Preserving Homeomorphisms of Open Surfaces of Genus Zero, New York Journal of Mathematics 2, pp 1-19.

[Gho] Ghomi, M., (2002) The problem of optimal smoothing for convex functions, Proc. Amer. Math. Soc., 130, pp 2255-2259. 
[Gho2] Ghomi, M., (2001) Strictly convex submanifolds and hypersurfaces of positive curvature, Journal of Diff. Geom., 57, pp 239-271.

[Hen] Henrard, J., (1999) The adiabatic invariant in classical mechanics, nonlinear and stochastic beam dynamics in accelerators - A challenge to theoretical and computational physics, v. 3 .

[Hi] Hirsch, M, (1976) Differential topology, Graduate Texts in Mathematics, $\mathrm{n}^{\circ}$ 33, SpringerVerlag, New York, Heidelberg.

[HK] Hofer, H., Kriener, M., Holomorphic curves in contact geometry, Proceedings of Symposia in Pure Math., Differential Equations, La Pietra 1996, Conference on Differential Equations, marking the 70th birthday of Peter Lax and Louis Nirenberg, v. 65, pp 77-132.

[HZW] Hofer, H., Wysocki, K., Zehnder, E., (1998) The dynamics on a strictly convex energy surface in $\mathbb{R}^{4}$, Ann. of Math. (2) 148, $\mathrm{n}^{\circ} 1$, pp 197-289.

[HZW2] Hofer, H., Wysocki, K., Zehnder, E., (2000) Pseudoholomorphic curves and dynamics in three dimensions, preprint.

[HZW3] Hofer, H., Wysocki, K., Zehnder, E., (1995) Properties of pseudoholomorphic curves in symplectisations II: embedding controls and algebraic invariants, GAFA, v. 5, pp270328.

[Ler] Lerman, L. M., (1991) Hamiltonian systems with loops of a separatrix of saddle center, Selecta Math. Sov. 10, 1991, pp 297-306.

[LK] Lerman, L. M., Koltsova, O. Yu., (1995) Periodic and homoclinic orbits in twoparameter unfolding of a Hamiltonian system with a homoclinic orbit to a saddle-center, Intern. Jour. Biff. Chaos 5, pp. 397-408.

[Mad] Maddocks, J. H., (1985) Restricted quadratic forms and their application to bifurcation and stability in constrained variational principles, SIAM J. Math. Anal., 16, pp 47-68.

[MHO] Mielke, A., Holmes, P., and O' Reilly, O., (1992) Cascades of homoclinic orbits to, and chaos near, a Hamiltonian saddle center, J. Dyn. Diff. Eqns. 4, pp. 95-126. 
[Mi] Milnor, J., (1965) Topology from the differential point of view, The University Press of Virginia, Char-lottesville.

[Mo] Moser, J., (1958) On the generalization of a theorem of A. Liapunoff, Comm. Pure Appl. Math. v. 11, pp. 257-271.

[McS] McDuff, D., Salamon, D., (1998) Introduction to symplectic topology, 2nd Edition. Oxford University Press.

[Rab] Rabinowitz, P., (1978) Periodic solution of Hamiltonian systems. Comm. Pure Appl. Math., 31, pp 157-184.

[Rag1] Grotta-Ragazzo, C., (1997) Irregular dynamics and homoclinic orbits to Hamiltonian saddle centers, Comm. Pure App. Math. L, pp 105-147.

[Rag2] Grotta-Ragazzo, C., (1997) On the stability of double homoclinic loops, Comm. Math. Phys, 184, pp 251-272.

[Russ] Russmann, H., (1964) Über das Verhalten analytischer Hamiltonscher differentialgleichungen in der Nähe einer Gleichgewichtslösung, Math. Ann. v. 154, pp. 285-300. Princeton University Press.

[Tho] Thorpe, J. , (1979) Elementary topics in differential geometry, Springer-Verlag, New York. 